\title{
The Use of Distributed Acoustic Sensing for 4D Monitoring Using Vertical Seismic Profiles: Results from the Aquistore $\mathrm{CO}_{2}$ Storage Project
}

\author{
by \\ Kyle Harris

\begin{abstract}
A thesis submitted to the
Faculty of Graduate and Postdoctoral Affairs

in partial fulfillment of the requirements for the degree of
\end{abstract}

Doctor of Philosophy in Earth Sciences

Department of Earth Sciences

Carleton University

Ottawa, Ontario

2017

CCopyright

Kyle Harris, 2017 


\section{Abstract}

Distributed acoustic sensing (DAS) is a rapidly developing technology that employs optical fibers to detect acoustic disturbances in the subsurface. The Aquistore $\mathrm{CO}_{2}$ storage project uses time-lapse DAS VSP's to monitor injected $\mathrm{CO}_{2}$ in a deep geological reservoir (>3200 m) beneath the study site near Estevan, Saskatchewan, Canada. Reservoir monitoring is a crucial component to any geological storage project, and aims to ensure the safe and effective containment of the injected fluids, and evaluate the integrity of the storage and sealing units.

An assessment of the ability of DAS VSP's to detect the $\mathrm{CO}_{2}$ response has been conducted for the Aquistore site. Prior to injection, fluid flow simulations were performed to predict $\mathrm{CO}_{2}$ distributions in the reservoir. These simulations were used to model the response of the $\mathrm{CO}_{2}$ plume in 2D time-lapse DAS VSP's. The expected responses were compared with estimates of time-lapse noise computed with field data from the baseline survey, and it was demonstrated that the plume would be visible in the reservoir after $27 \mathrm{kt}$ of injection.

After $36 \mathrm{kt}$ of $\mathrm{CO}_{2}$ injection, the first monitor dataset was acquired. 4D VSP processing and imaging were applied to produce time-lapse difference volumes of the reservoir. Acceptable repeatability was attained, with normalized root-mean-square (nRMS) values less than 0.4 beneath the observation and injection wells. An anomaly in the lower part of the reservoir near the observation and injection wells was attributed to the replacement of brine with $\mathrm{CO}_{2}$. 
Updated fluid flow simulations were obtained that better replicated the injection parameters observed at the study site. Forward seismic modeling was then performed for $36 \mathrm{kt}$ and $97 \mathrm{kt}$ injection scenarios that reflected the 3D shot and receiver geometry in the field acquisitions. These data were processed using the same sequence applied to the field data to obtain comparable time-lapse difference volumes. Comparisons between the field and synthetic $36 \mathrm{kt}$ anomalies were used to refine interpretations of the $\mathrm{CO}_{2}$ distribution in the reservoir. However, they also revealed the need to update the geological model to better reproduce the laterally asymmetric plume expansion. 


\section{Acknowledgements}

Foremost, I thank my PhD co-supervisors Dr. Don White and Dr. Claire Samson. Without their guidance, expertise, and encouragement this dissertation would not have been possible. I would also like to thank everyone at (or formally at) the 3D Imaging and Earth Modelling group at the Geological Survey of Canada, especially Gilles, Lisa, Brian, and Dave. Their willingness to advise me throughout this research was always appreciated, and their input was always valuable. I also appreciate the support from the Earth Sciences department at Carleton University, and I am deeply grateful for the Research Affiliate Program (RAP) bursaries made available by Natural Resources Canada.

Finally, I would like to express my gratitude to my parents and to Carolyn for providing encouragement and support throughout these four years. 


\section{Preface}

This document is an integrated thesis consisting of three articles published or submitted to peer-reviewed scientific journals on the topic of distributed acoustic sensing for 4D reservoir monitoring:

1. Harris, K., White, D. Melanson, D., Samson, C., and Daley, T., 2016, Feasibility of time-lapse VSP monitoring at the Aquistore $\mathrm{CO}_{2}$ storage site using a distributed acoustic sensing system: International Journal of Greenhouse Gas Control, 50, 248-260 (doi: 10.1016/j.ijggc.2016.04.016). Published [Chapter 5]

2. Harris, K., White, D., and Samson, C., 2017, Imaging the Aquistore reservoir after 36 kilotonnes of $\mathrm{CO}_{2}$ injection using distributed acoustic sensing: Geophysics, 82 (6), M81-M96 (doi: 10.1190/geo2017-0174.1). Published [Chapter 6]

3. Harris, K., White, D., Samson, C., and Jiang, T., 201x, 4D vertical seismic profile modeling of $\mathrm{CO}_{2}$ injection scenarios at Aquistore: submitted to the International Journal of Greenhouse Gas Control. [Chapter 7]

Each article has been modified to comply with input from the thesis examination committee. Table and figure numbers have been standardized and updated to be consistent within the thesis, and a list of references has been compiled at the end.

In paper 1, K. Harris wrote the manuscript, developed the 2D migration software, and performed the seismic processing and repeatability analysis. D. White planned the seismic surveys, assisted in establishing the research objectives, advised on the software development, and provided extensive comments on the article and response to reviewers' 
comments. D. Melanson performed the fluid-replacement modeling and seismic simulations used to estimate the $\mathrm{CO}_{2}$ plume response. C. Samson assisted in establishing the research objectives and provided valuable comments on the manuscript. T. Daley assisted in the DAS data acquisition and the preparation of the DAS data. The fluid flow simulations were conducted by the Energy and Environmental Research Center (EERC), as was the construction of the geostatic model.

In paper 2, K. Harris wrote the manuscript, developed and implemented the processing flow, and characterized the plume response. D. White planned the seismic surveys, advised on the development of the processing flow and plume response characterization, and provided extensive comments on the article and response to reviewers' comments. C. Samson provided valuable comments on the manuscript.

In paper 3, K. Harris wrote the manuscript, performed fluid-replacement modeling and seismic simulations, developed and implemented the processing flow, and characterized the plume response. D. White assisted in establishing the research objectives, planned the seismic surveys, advised on the development of the processing flow and plume response characterization, and provided extensive comments on the article. C. Samson provided valuable comments on the manuscript. T. Jiang (EERC) performed the fluid flow simulations that were used to build the seismic models.

The thesis co-supervisors, Prof. Claire Samson and Dr. Don White, acknowledge the above information as accurate. 


\section{Table of Contents}

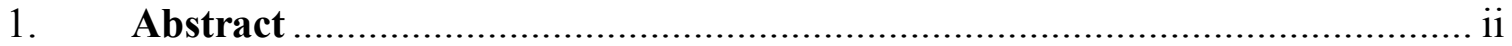

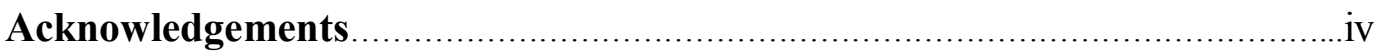

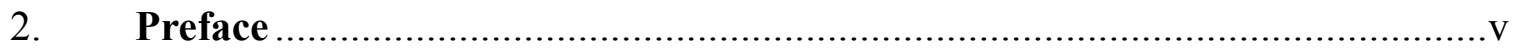

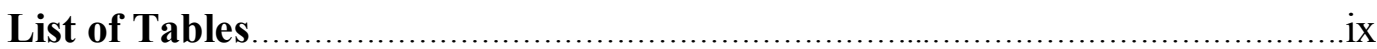

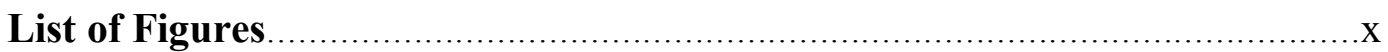

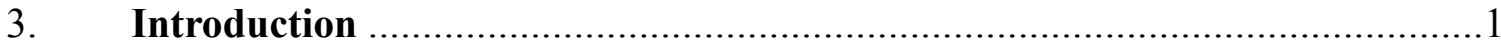

3.1. Aquistore carbon storage project ............................................................

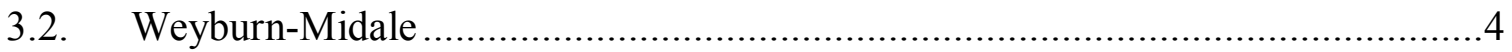

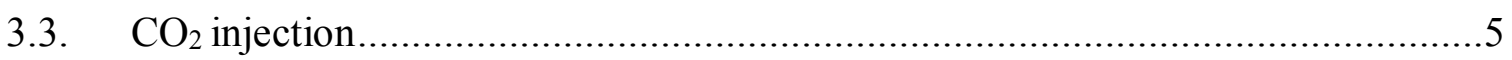

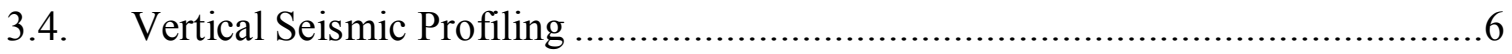

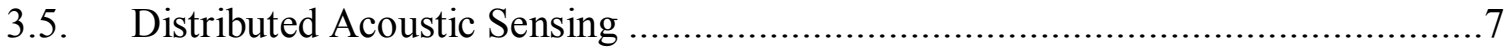

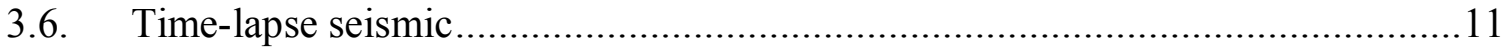

3.7. Aquistore seismic monitoring background..............................................13

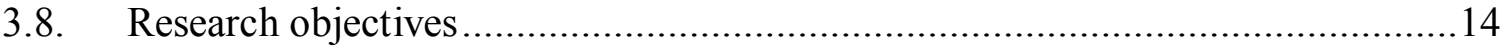

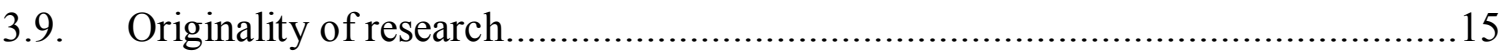

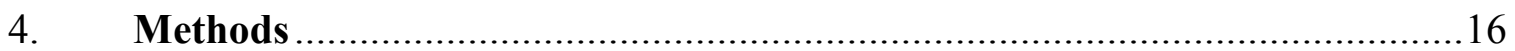

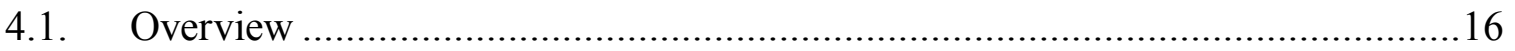

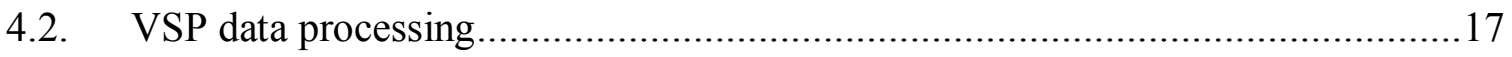

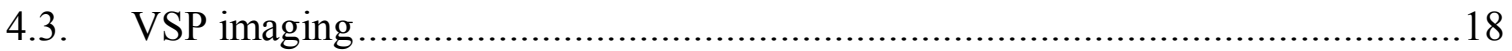

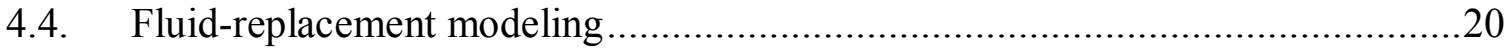

4.5. Seismic modeling with finite differences ...................................................22

5. Feasibility of Time-lapse VSP Monitoring at the Aquistore $\mathrm{CO}_{2}$ Storage Site Using a Distributed Acoustic Sensing System .......................................24

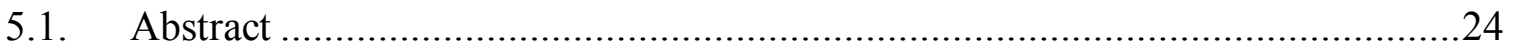




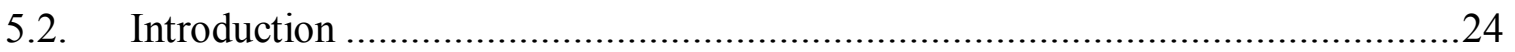

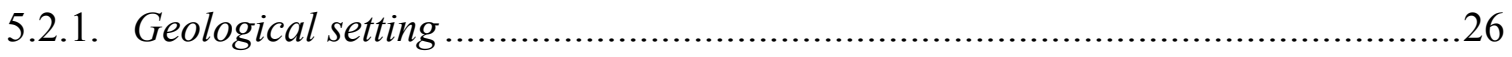

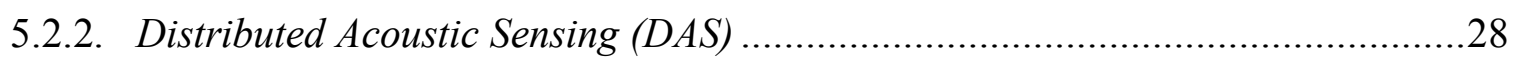

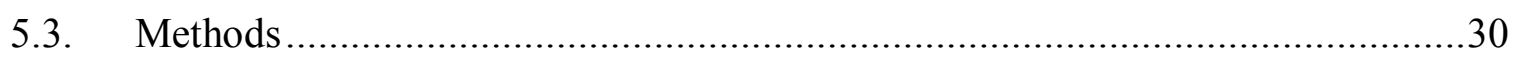

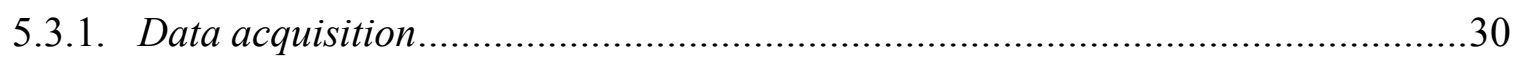

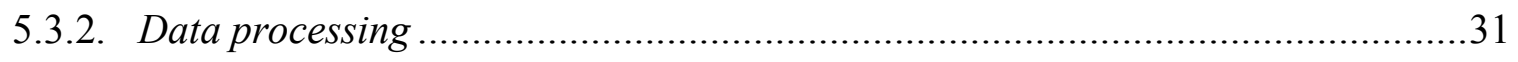

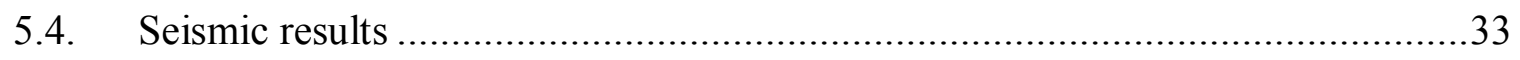

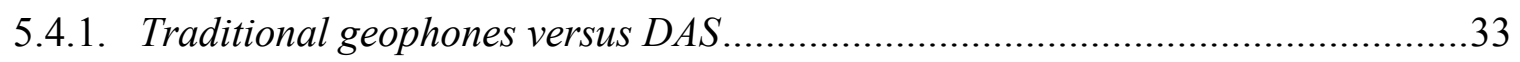

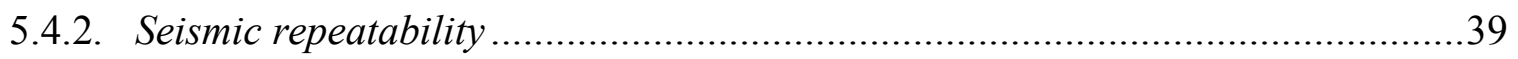

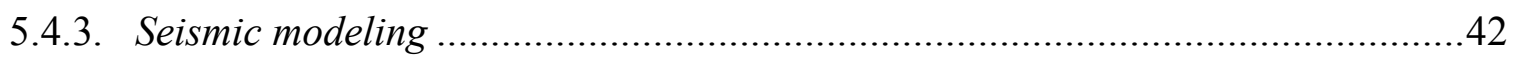

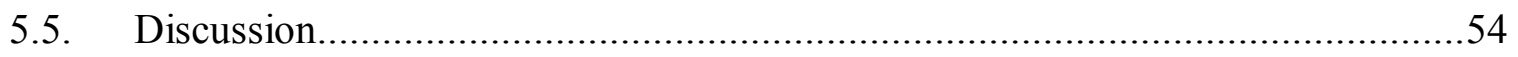

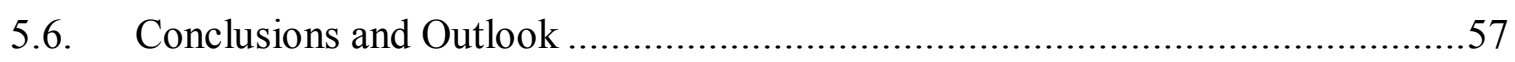

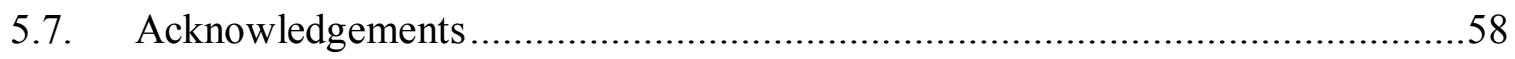

6. Imaging the Aquistore Reservoir after 36 kilotonnes of $\mathrm{CO}_{2}$ Injection using Distributed Acoustic Sensing.............................................................................59

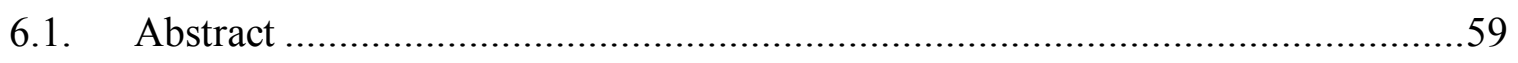

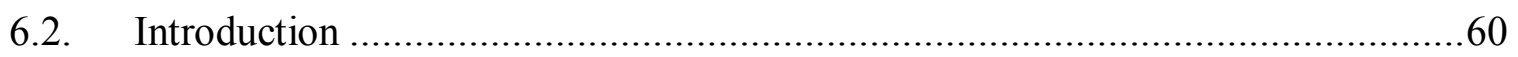

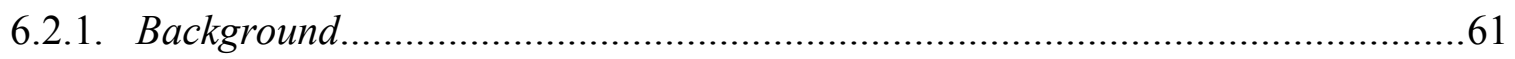

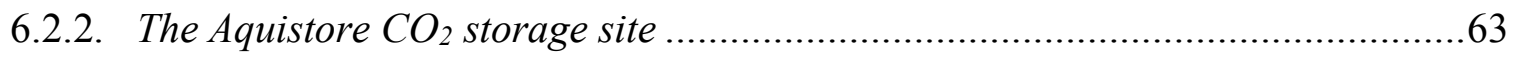

6.2.3. Distributed Acoustic Sensing......................................................................65

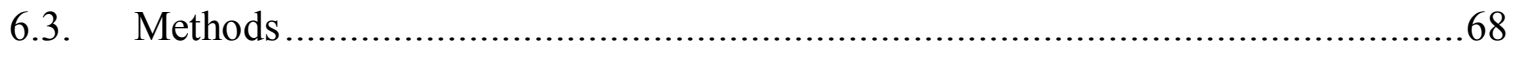

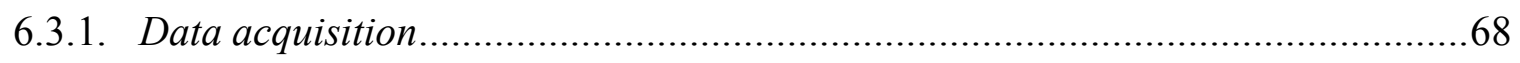

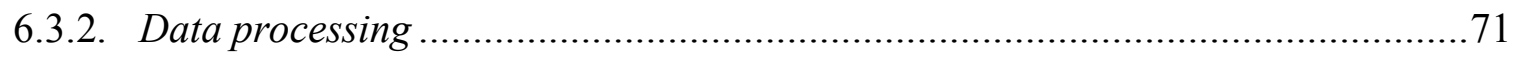

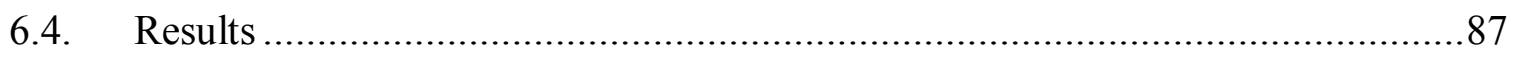

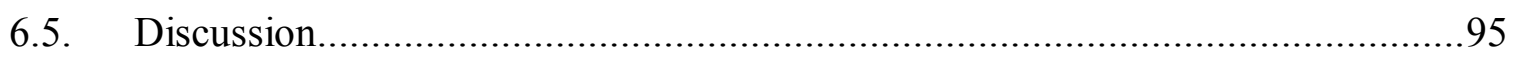




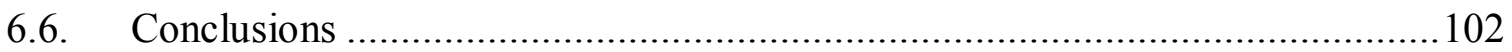

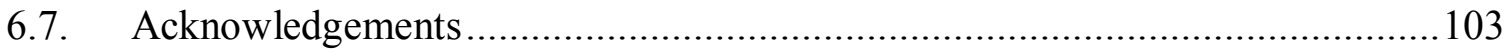

7. 4D Vertical Seismic Profile Modeling of $\mathrm{CO}_{2}$ Injection Scenarios at Aquistore

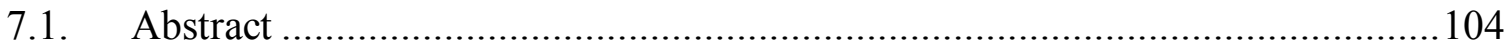

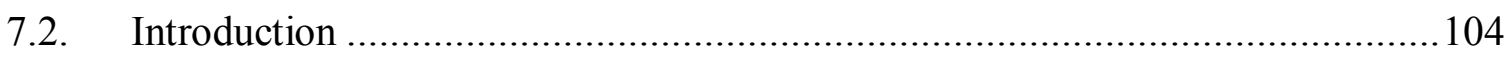

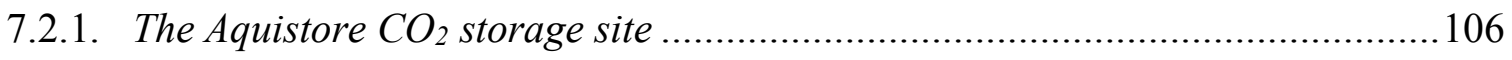

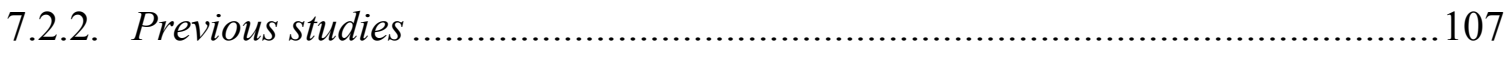

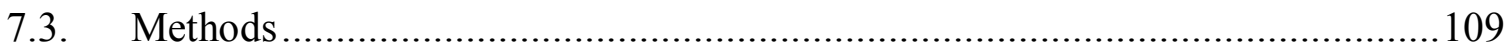

7.3.1. Geological model and fluid flow simulations ....................................................109

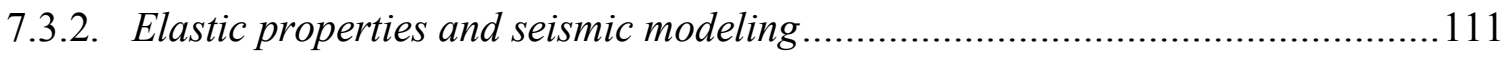

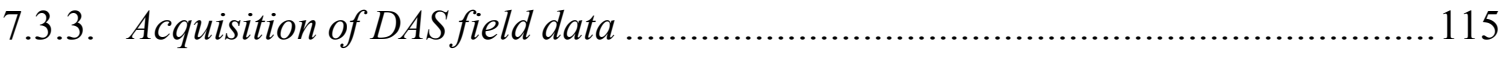

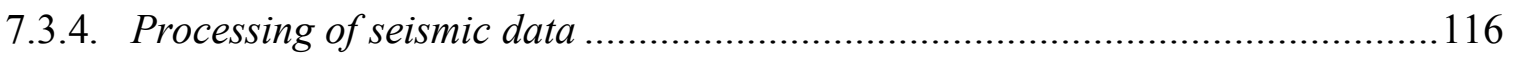

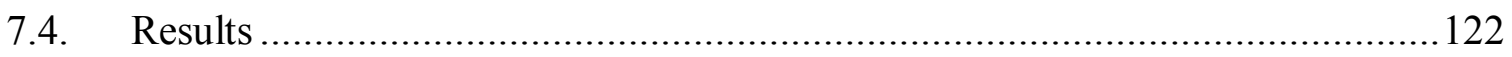

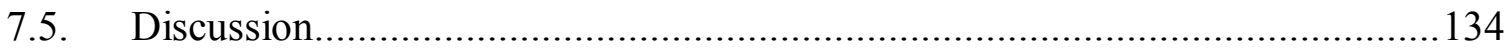

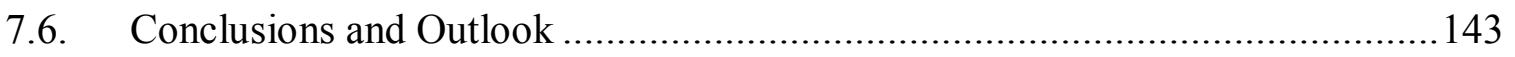

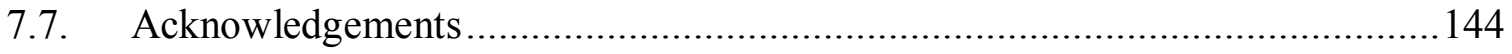

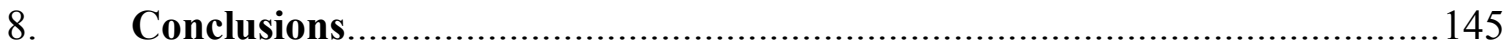

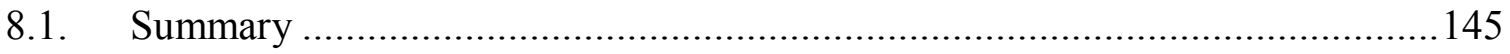

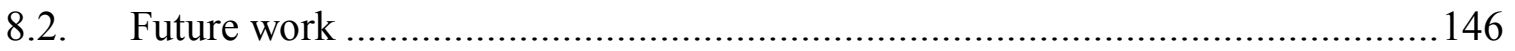

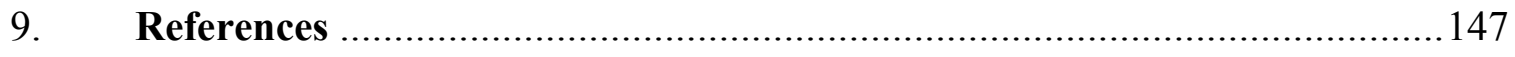




\section{List of Tables}

Table 5-1: Petrophysical properties of reservoir units at Aquistore based on injection well

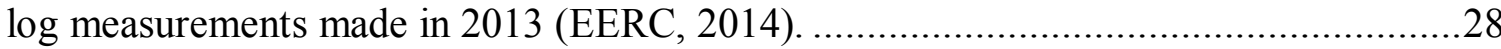

Table 5-2: Finite Difference Model Parameters (Modified from Melanson, 2015).........46

Table 6-1: Acquisition parameters for baseline and monitor DAS surveys ...................69

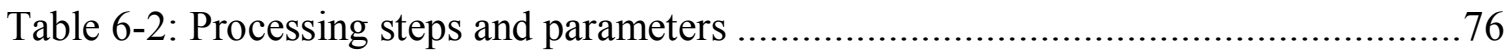

Table 7-1: Finite Difference Model Parameters................................................... 114

Table 7-2: Acquisition parameters for baseline and monitor DAS surveys ..................116

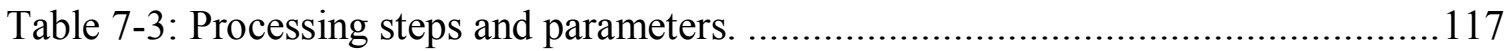




\section{List of Figures}

Figure 3-1: Stratigraphic column of Williston basin at Aquistore injection well. .............3

Figure 3-2: Result of a fluid flow simulation in the Aquistore reservoir ........................

Figure 3-3: Source-receiver geometry of a vertical seismic profile ............................6

Figure 4-1: Generalized VSP imaging workflow .................................................... 19

Figure 5-1: Location map of the Aquistore $\mathrm{CO}_{2}$ storage site..................................26

Figure 5-2: Comparison of raw vertical-component geophone data and single-mode DAS data for a single shot gather (direct comparison) ..................................................33

Figure 5-3: Comparison of raw DAS seismic data and vertical-component geophone data

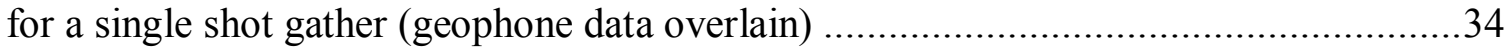

Figure 5-4: Signal and noise power spectra for geophone data and DAS ......................36

Figure 5-5: 8-fold migrated images of the single-mode DAS and vertical-component geophone VSP data with migrated section of surface data.........................................38

Figure 5-6: $1000 \mathrm{~m}$ offset shots from the November 2013 pre-injection baseline survey

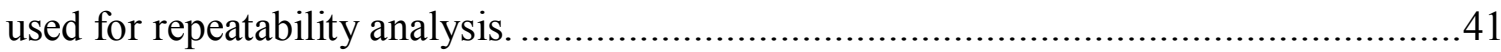

Figure 5-7: $50 \mathrm{~m}$ window nRMS map of amplitude differences between shots 45364016 and 45603968 of the November 2013 pre-injection baseline survey.....

Figure 5-8: Modeled $\mathrm{CO}_{2}$ saturation in reservoir after 90 days, 1 year, 2 years, and 3 years

of $\mathrm{CO}_{2}$ injection at a rate of $301 \mathrm{t} /$ day .45

Figure 5-9: DAS $400 \mathrm{~m}$ offset shot gather recorded in the field versus equivalent

synthetic shot gather

Figure 5-10: Comparison of migrated 50-shot stack of baseline FDTD synthetic data and

field data. 
Figure 5-11: Migrated synthetic time-lapse difference images after 90 days, 1 year, 2 years, and 3 years of $\mathrm{CO}_{2}$ injection at a rate of $301 \mathrm{t} /$ day .52

Figure 5-12: $10 \mathrm{~m}$ window nRMS maps of the amplitude residuals of the time-lapse difference images shown in Figure 5-11...... .53

Figure 5-13: 10 m window RMS maps of the amplitude residuals of the time-lapse

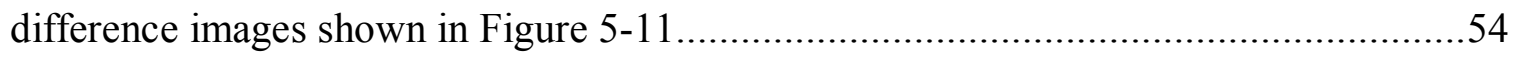

Figure 6-1: Location map of the Aquistore $\mathrm{CO}_{2}$ storage site and survey area................61

Figure 6-2: Corridor stack of near-offset DAS shot gather and injection well logs ........65

Figure 6-3: VSP baseline shot locations ........................................................ 71

Figure 6-4: Examples of spectra for two shot gathers from the baseline and monitor surveys .74

Figure 6-5: Examples of shot gathers from the baseline and monitor surveys exhibiting good and poor repeatability. .75

Figure 6-6: Examples of amplitude-equalized shot gathers for shot locations that are different between the baseline and monitor surveys .78

Figure 6-7: Abrupt amplitude and $\mathrm{S} / \mathrm{N}$ decay of first arrivals at a depth of approximately $1000 \mathrm{~m}$ for a near-offset shot gather.

Figure 6-8: f-k spectrum of shot gather 46323812 from the monitor survey 80 Figure 6-9: Anisotropic P-wave velocity model used for migration. .82 Figure 6-10: The expected position of the $\mathrm{CO}_{2}$ plume in relation to the 4D VSP zone of specular illumination .84

Figure 6-11: Stacks of the 9 monitor shot gathers that are closest to the observation well, depth migrated with a narrow aperture or a wide aperture 84 
Figure 6-12: Plan view of the nRMS difference image for a horizon within the upper Deadwood interval of the reservoir

Figure 6-13: Fold map for 50-shot subset at reservoir top for specular reflections and Kirchhoff migration with $\pm 15^{\circ}$ aperture

Figure 6-14: Baseline, monitor, and difference of shot gather 46323812 after removal of near-surface traces, application of a Butterworth filter (10-80 Hz, $6 \mathrm{~dB}$ roll-off), $\mathrm{t}^{2}$ amplitude scaling, depth alignment, and amplitude cross-equalization .88

Figure 6-15: Baseline, monitor and difference shot gathers 46323812 at progressive stages in the processing sequence .90

Figure 6-16: Histograms of nRMS values for time-lapse difference images before and after post-migration cross-equalization

Figure 6-17: nRMS cross-sections immediately above reservoir depth before and after post-migration cross-equalization.

Figure 6-18: Amplitude cross-section of baseline, monitor, and difference depth-migrated volumes intersecting the observation well. .93 Figure 6-19: Values of nRMS of monitor-baseline differences for a $75 \mathrm{~m}$ window in cross-sections through observation and injection wells

Figure 6-20: Plan view of nRMS difference images for the reservoir caprock and 3 intervals where $\mathrm{CO}_{2}$ is injected .96

Figure 6-21: nRMS vs RMS comparison for a cross-section through the injection well computed with a $75 \mathrm{~m}$ window

Figure 6-22: Cross-section of nRMS values for migrated synthetic data after 27 ktonnes of injected $\mathrm{CO}_{2}$ 
Figure 6-23: Plan view of nRMS difference images in the upper Deadwood showing VSP result and surface-based result .100

Figure 7-1: Curve of cumulative injected $\mathrm{CO}_{2}$ versus time used in fluid flow simulations. 106

Figure 7-2: Location of Aquistore $\mathrm{CO}_{2}$ storage site and seismic survey area 107

Figure 7-3: Plan view $34 \mathrm{~km}^{2}$ fluid simulation grid .110 Figure 7-4: Baseline 1-D velocity and density model used as input for forward seismic modeling for the $0 \mathrm{kt}$ injection scenario. .115

Figure 7-5: Example of a near-offset synthetic shot gather progressing through the processing sequence

Figure 7-6: Continued example of a near-offset synthetic shot gather progressing through the processing sequence

Figure 7-7: South-north cross-sections of the reservoir through the injection well showing saturation and $\mathrm{P}$-wave velocity difference from baseline, and plan view of $\mathrm{P}$-wave velocity at $3300 \mathrm{~m}$ 123

Figure 7-8: Changes in P-wave velocity, density, P-wave acoustic impedance (AI), and Swave velocity as a function of $\mathrm{CO}_{2}$ saturation for each zone located adjacent to a well perforation zone. .126 Figure 7-9: Comparison of near-offset shot gathers for the baseline and $36 \mathrm{kt}$ injection scenarios. 127 Figure 7-10: Comparison of migrated 50-shot stack of baseline FDTD synthetic data and field data .129 
Figure 7-11: West-east cross-sections through the injection well for $36 \mathrm{kt}$ synthetic data, and $97 \mathrm{kt}$ synthetic data. Sections show $\mathrm{CO}_{2}$ saturations predicted from the flow simulations, and nRMS of the time-lapse seismic differences in the reservoir. 130

Figure 7-12: West-east cross-sections through the injection well for $36 \mathrm{kt}$ field data, $36 \mathrm{kt}$ synthetic data, and $97 \mathrm{kt}$ synthetic data. Sections show raw time-lapse differences, RMS differences, and nRMS of the differences in the reservoir 132 Figure 7-13: Depth shifts between baseline and $97 \mathrm{kt}$ synthetic data for horizons above reservoir and below reservoir, and nRMS anomaly below reservoir 133 Figure 7-14: 10 m-thick depth sections through the RMS and nRMS difference volumes in the lower Deadwood 134 Figure 7-15: The effect on reflectivity of using the total versus effective porosity in the Gassmann methodology 138

Figure 7-16: $\Delta \mathrm{Vp}$ sensitivity plots for log-based parameters in Gassmann fluid substitution calculations 140 


\section{Introduction}

The work undertaken in this thesis combines two emerging fields: carbon capture and storage (CCS) in a geological reservoir; and distributed acoustic sensing (DAS). Though there had been a considerable amount of prior experience in subsurface injection of $\mathrm{CO}_{2}$ for the purpose of enhanced oil recovery (EOR), the first CCS project commenced injection in the fall of 1996 in the Sleipner oil field in Norway (Arts et al., 2008). Sleipner and many subsequent CCS projects employ well-logging techniques and timelapse seismic measurements to monitor the migration of $\mathrm{CO}_{2}$ in the subsurface. Examples

include plume monitoring with surface arrays in In Salah, Algeria (Ringrose et al., 2013) and cross-well seismic tomography in Nagaoka, Japan (Saito et al., 2008). A common approach included in modern integrated CCS monitoring plans is vertical seismic profiling (VSP), as employed in Decatur (Couëslan et al., 2013), Otway (Correa et al., 2017), Weyburn-Midale (Ahmadi et al., 2011), Ketzin (Götz et al., 2014), and Quest (Bacci et al., 2017). The numerous advantages of using VSPs for reservoir monitoring are discussed in section 5.2, but the method is also associated with higher costs and efforts.

\subsection{Aquistore carbon storage project}

Aquistore is an independent research and monitoring project managed by the Petroleum Technology Research Centre (PTRC), Regina, Saskatchewan. The project aims to demonstrate the feasibility of storing $\mathrm{CO}_{2}$ in a deep sandstone formation as a safe method of reducing greenhouse gas emissions in the atmosphere. The Aquistore site acts as the carbon storage location for the SaskPower Boundary Dam Integrated Carbon Capture and Storage Demonstration Project, and is located southwest of the town of 
Estevan, Saskatchewan. The Boundary Dam project is the first fully integrated CCS demonstration project in the world that uses emissions from a coal-fired power plant, and Aquistore is the first project solely dedicated to $\mathrm{CO}_{2}$ storage in Canada (Worth et al., 2014).

The Unit 3 turbine at the Boundary Dam plant outputs $150 \mathrm{MW}$ of power with 40 MW required for the CCS processes, which capture $90 \%$ of the $\mathrm{CO}_{2}$ produced. Most of the captured $\mathrm{CO}_{2}$ is allocated for enhanced oil recovery at the Cenovus Energy Weyburn oilfield, leaving $\sim 110 \mathrm{kt}$ that has been injected at Aquistore as of October 2017. The injection target consists of the Deadwood Formation and Black Island member of the Winnipeg Formation (Figure 3-1). Together these comprise the Cambro-Ordovician flow unit within the Williston Basin. These units contain alternating layers of sandstones and shales that will result in a stratified $\mathrm{CO}_{2}$ distribution in the reservoir (e.g. Figure 3-2). The thickness of the sandstone layers varies between 6-50 m, with the thickest at the bottom of the Deadwood. The primary sealing unit is the $15 \mathrm{~m}$ thick Ice Box shale at the top of the Winnipeg formation, and the secondary sealing unit is the $150 \mathrm{~m}$ thick Prairie Evaporite located $500 \mathrm{~m}$ above the reservoir. 3D seismic images and well-logs have been used to investigate the suitability of the storage complex prior to injection (Rostron et al., 2014; White et al., 2016). This analysis has shown that the reservoir is continuous with highly-permeable storage intervals, and that the sealing formations are continuous and contain no significant faults in the storage area. Additional site characterization has also shown that the groundwater and soil gas horizons near the surface do not appear to be hydraulically connected to the injection intervals. 


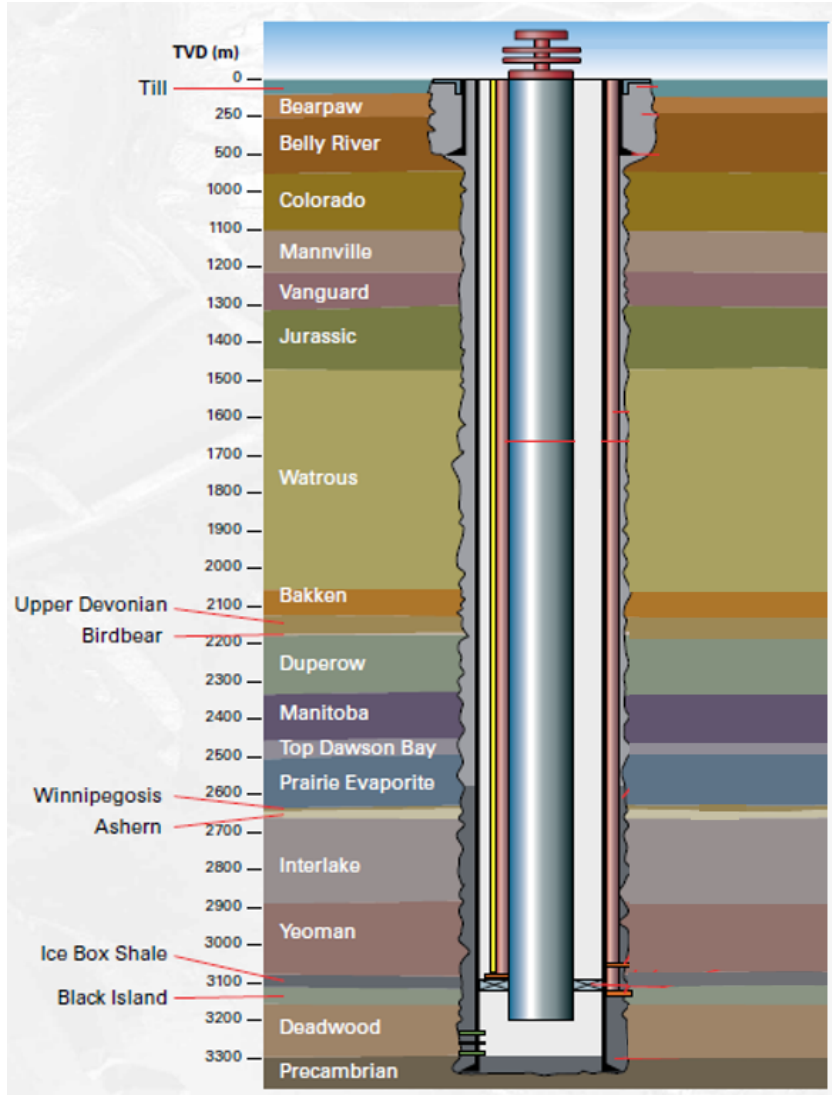

Figure 3-1: Stratigraphic column of the Williston basin at the Aquistore injection well (credit:

Schlumberger Carbon Services).

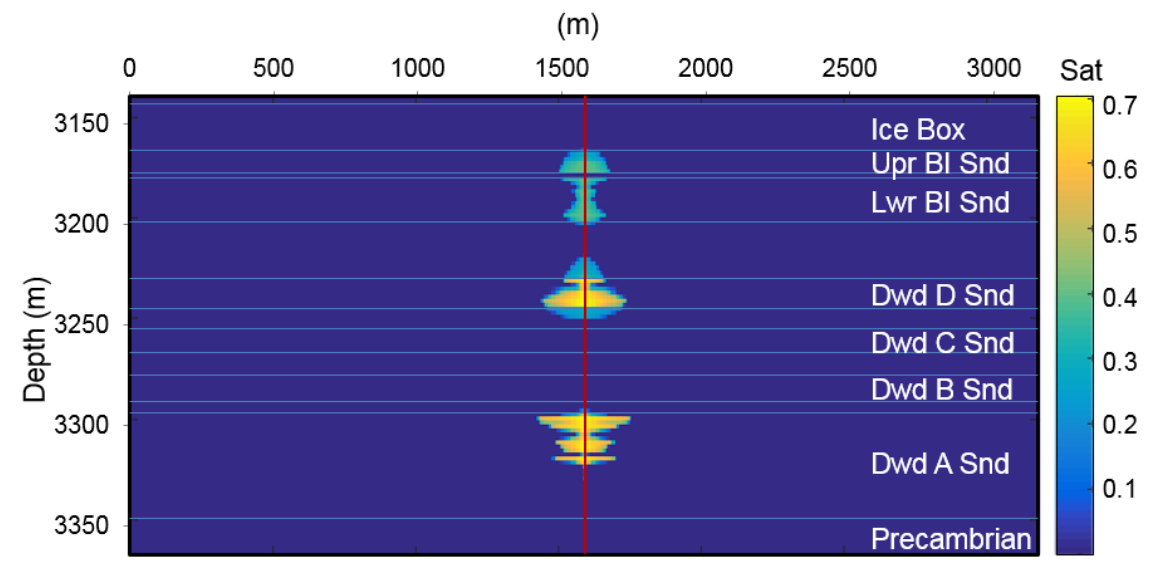

Figure 3-2: Result of a fluid flow simulation in the Aquistore reservoir showing $\mathrm{CO}_{2}$ saturations. $\mathrm{CO}_{2}$ distribution is stratified into sandstone units (Upper and Lower Black Island, Deadwood A, B, C, and D) separated by thin shale units. 


\subsection{Weyburn-Midale}

Also located in the Williston Basin, the Weyburn-Midale $\mathrm{CO}_{2}$ Monitoring and Storage Project is of particular relevance to the research undertaken here. Aquistore (the second flagship project of the Petroleum Technology Research Centre) was planned and operated, in large part, with the experience gained directly from Weyburn-Midale.

Cenovus has been injecting $\mathrm{CO}_{2}$ at Weyburn since 2000, while Apache Canada has been extensively injecting at Midale since 2005 (it should be noted that Shell had piloted $\mathrm{CO}_{2}$ injection at Midale as far back as the 1980's). Combined, they had attained an injection rate of $\sim 3 \mathrm{Mt} /$ year in 2012 (Verdon, 2012). The $\mathrm{CO}_{2}$ is injected along with water at a depth of $\sim 1500 \mathrm{~m}$ into the two reservoirs, and the project marks the first time that an anthropogenic source of $\mathrm{CO}_{2}$ has been used for enhanced oil recovery. The first phase of the Weyburn-Midale project was completed in 2004, and sought to verify that secure and economical containment of $\mathrm{CO}_{2}$ in the Weyburn reservoir was possible. The goal of the second phase, completed in 2011, was to expand upon the first and to establish a framework for future $\mathrm{CO}_{2}$ geological storage projects.

One focus of the Weyburn-Midale project was the development of monitoring methods to effectively track $\mathrm{CO}_{2}$ migration in the subsurface. This ability was clearly demonstrated via the use of 3D time-lapse seismics by White (2009). The study found that the areal extent of time-lapse anomalies in the injection intervals were directly related to the cumulative amounts of $\mathrm{CO}_{2}$ injected. Control horizons above the reservoir were also monitored, and exhibited low background noise and good repeatability, thus contributing to the robustness of the delineation of the plume-based anomalies. Certain findings from the surface seismic monitoring at Weyburn guided efforts for VSP 
monitoring at Aquistore. For example, though P-wave time-lapse images were shown to be effective at mapping locations where $\mathrm{CO}_{2}$ is present, they were relatively insensitive to increases at higher saturations. Therefore volume estimates from seismic data could only be made in association with accurate reservoir flow simulations. Consequently, the work presented in this thesis employs reservoir simulations to make such interpretations (Chapters 5 and 7), and to establish the feasibility of $\mathrm{CO}_{2}$ detection.

\section{3. $\mathrm{CO}_{2}$ injection}

During injection, the pressure around the well increases and allows the $\mathrm{CO}_{2}$ to displace the in-situ brine in the reservoir. This pressure increase is controlled primarily by the injection rate, and the permeability and thickness of the injection formation. $\mathrm{CO}_{2}$ enters the saline formation in a supercritical state $\left(>31.10^{\circ} \mathrm{C}\right.$ and $\left.>7.39 \mathrm{MPa}\right)$, and has a much higher mobility than the mutually immiscible in-situ brine (Celia et al., 2005). This leads to only a partial displacement of the brine, with resulting $\mathrm{CO}_{2}$ saturations in the range of 30-60\% near the injection well (IPCC, 2005). Typically, the volumetric rate of brine displacement is much lower than the $\mathrm{CO}_{2}$ injection rate due to compressibility effects and small amounts of leakage into the sealing shale units (Birkholzer et al., 2009).

After injection, the $\mathrm{CO}_{2}$ continues to migrate through the formation and slowly dissolves $\left(10^{1}-10^{2}\right.$ years $)$ into the brine. Once dissolved, the $\mathrm{CO}_{2}$ migrates along with the regional groundwater flow. For deep basins, flow is usually on the order of mm-cm/year, which is much slower than mechanisms that would drive the movement of separate-phase $\mathrm{CO}_{2}$ (Bachu et al., 1994). 


\subsection{Vertical Seismic Profiling}

A VSP is a seismic measurement procedure that includes one or more surface-located shots recorded by sensors deployed at various depths in the subsurface (Figure 3-3). The plane of the shot grid is thus oriented $90^{\circ}$ from the receiver array (Hardage, 2000).

Exceptions include cases of deviated well paths or dipping surface topography.

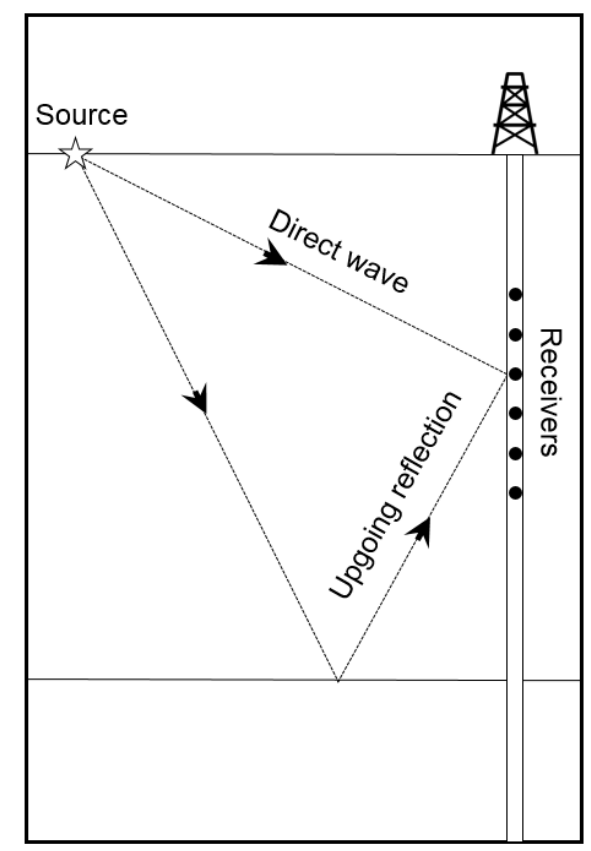

Figure 3-3: Source-receiver geometry of a vertical seismic profile

Receivers located at depth are capable of recording both the downgoing and reflected upgoing wavefields. Generally, this allows for several advantages over surface seismic methods: depth registration of primary reflections, identification of intrabed multiple reflections, measurement of vertical P- and S-wave velocities, characterization of wave mode conversions, and deconvolution of the reflected wavefield from the downgoing wavefield. VSPs also provide better resolution than surface surveys as a result of the paths traveled by the waves. In surface seismic surveys, the waves must travel twice 
through highly weathered layers near the surface, thus greatly attenuating high frequencies. Waves in VSP surveys only travel through these layers once.

Until the early 1980 's, the only downhole recording tools available were single geophone assemblies that required repositioning between each of many shots to obtain a full vertical profile (Hardage, 2000). Presently, conventional geophone deployments rarely exceed 100 levels in a single tool, and 25 in a slim borehole, and each level must independently deploy a coupling mechanism. These limitations may be mitigated with the use of the optical fiber-based DAS system in place of conventional geophones.

\subsection{Distributed Acoustic Sensing}

The practicality of fiber optic cables in seismic surveying (particularly in geophysical surveillance and in-well monitoring) has greatly improved in recent years when the need for discrete point sensors was removed by using the fiber itself as the sensor. This allows the fiber to be employed as a continuous array of sensors, and is the principle behind DAS. Unlike the discrete sensor systems, DAS does not require manufactured sensors, nor does it require multiple fibers or optical multiplexing (Miller et al., 2012). Nearly any optical cable can be used as a distributed array acoustic sensor, and can remain installed in a downhole environment for years (Mestayer et al., 2011). The DAS technology offers a reduced cost alternative to the traditional geophone methods, as each repeat DAS survey will require only new seismic sources and equipment, whereas temporary deployment of geophones downhole via wireline can be very costly. Methodology for installing optical cables down boreholes has already been well established for distributed temperature sensing (DTS). DTS requires multi-mode fiber whereas DAS can use either multi-mode or single mode fiber. Multiple fibers may 
be easily deployed in a single capillary tube, thus it is convenient to use DAS and DTS in parallel (Daley et al., 2013; Mestayer et al., 2011).

DAS systems operate by pulsing light down an optical fiber and detecting the resulting backscattered signal. Variations in signal can be used to characterize the localized strain imparted on the fiber by a seismic wavefield. This requires that the fiber is coupled in some way, via friction or pressure, with the surrounding wavefield to generate longitudinal strain on the fiber. The Silixa iDAS system uses a single pulse to measure the change in optical path length between two sections of the fibre, providing an estimate of the strain rate. The distance that separates the two sections is chosen by the operator, and is termed "gauge-length". A different method is employed in the Optasense $\mathrm{ODH}$ system, which requires two pulses for a single measurement. Here the gauge-length is necessarily the distance between two successive pulses (also chosen by the operator), and the phase of the interference signal can be converted to the optical path length change in the fiber (Hornman, 2016). This type of system provides an estimate of the fibre strain.

Regardless of the type of system employed, DAS is effectively sensitive to strain primarily along the longitudinal axis of the fibre. Though advances in improving the directionality are underway (Hornman, 2016), the technology in its present state can only yield data comparable to that of vertical-component geophones. Prior to the Aquistore project, several field tests of DAS have been performed, including comparisons with conventional geophones.

Shell first tested DAS as a proof of concept for seismic monitoring in September 2009 in a tight (low porosity silt/sand) gas reservoir. The study demonstrated the 
capability of the technology to record down-hole seismic data from active sources (vibroseis and dynamite). In 2011, Mestayer and co-workers, in collaboration with Shell, conducted a study to evaluate the viability of VSP (in particular of DAS data) as a measurement monitoring tool for the Quest Carbon Capturing and Sequestration Project. The project intended to capture 1.2 million tones of $\mathrm{CO}_{2}$ annually from the Scotford Upgrader in Alberta, Canada and transport it via a pipeline to underground storage. The study evaluated several different monitoring methods, including zero-offset VSP to monitor $\mathrm{CO}_{2}$ containment, walk-away VSP to track the injected $\mathrm{CO}_{2}$ plume, and tube wave monitoring of casing integrity. The study showed that in the zero-offset tests, reflections were interpretable down to $2 \mathrm{~km}$, and that time-lapse 3D VSP of shallow (down to $2 \mathrm{~km}$ ) target geometries is feasible. The noise present in the DAS data was believed to be inherent to the instrument, as it was roughly constant with depth, and the signal fell below the noise floor at depths greater than $4 \mathrm{~km}$. The noise floor was significantly higher than in the traditional geophone data. Mestayer and co-workers (2011) also suggested that the savings in time and effort could be used towards high fold shot stacking for improved results. However, it was found that the walk-away VSP data was nearly equivalent to the traditional geophone data with regards to signal to noise ratio (SNR) and resolution. The study concluded that DAS is not only suitable for reservoir monitoring applications, but that there are situations where it is the only viable alternative for down-hole geophysical surveillance, such as in slim hole wells, in holes that cannot be entered without removing completion equipment, or where long geophone arrays cannot be easily deployed (long horizontal wells) (Mestayer et al., 2011). Cox and coworkers (2012) later constructed time-lapse images of the subsurface at Quest from the 
walk-away VSP's, and achieved excellent repeatability $(\mathrm{nRMS}=0.15)$ at depths up to $2200 \mathrm{~m}$. The study used a repeat survey that was acquired three days after the baseline survey, reflecting ideal conditions for repeatability.

In a 2013 study, Daley and co-workers conducted field tests at three locations to examine the applications of the Silixa iDAS system in borehole and surface measurements. In these early tests, iDAS was a prototype DAS system, and each test was part of a $\mathrm{CO}_{2}$ storage monitoring pilot project.

The first test was at an oilfield in Citronelle, Alabama operated by Denbury Resources. The sequestration project was operated by Southeast Carbon Sequestration Partnership. A walk-away VSP was conducted at this site in 2012, where it was found that P-wave energy was easily detectable at a depth of $2.1 \mathrm{~km}$ (Daley et al., 2013). A subsequent study undertook a comparison of fibre optic and geophone seismic data from a VSP acquisition. The study saw a clear improvement in SNR from the previous acquisition, and was attributed to improved recording procedures, DAS acquisition hardware, and processing. Though the SNR in the DAS data was $18-24 \mathrm{~dB}$ below that of equivalent geophone data, the study concluded that the extra source effort required to improve the DAS SNR is well compensated by the finer spatial sampling and lower deployment cost (Daley, 2016).

The second site used in the iDAS tests was located near Warrnambool, Victoria, Australia, as part of the Otway sequestration pilot operated by the CO2CRC research organization. The optical fiber was deployed downhole in a manner similar to that at Citronelle, but a higher energy weight drop source was employed. A fiber was also 
deployed along the surface with the receiver line orthogonal to the source line.

Downgoing and upgoing P-wave energy could be clearly identified in the VSP data, however, the SNR was consistently inferior to that of the traditional geophones at all depths. Signal amplitudes were found to significantly decay at offsets greater than $400 \mathrm{~m}$, and strong ground roll was observed. A subsequent study (Correa et al., 2017) presented preliminary monitoring results after 5,10 , and $15 \mathrm{kt}$ of $\mathrm{CO}_{2} / \mathrm{CH}_{4}$ was injected into a $\sim 1500 \mathrm{~m}$ deep reservoir. The study found that main reflectors could be identified, but noise was still strong, and that there was a considerable azimuth dependence on DAS sensitivity.

The third site was located at a pilot storage site at Ketzin, Germany operated by the German Research Centre for Geosciences. The optical fiber was deployed down-hole behind casing and a zero-offset VSP survey was conducted. The downgoing P-wave was clearly visible, along with many reflections, and was consistent with what the traditional geophone VSP recorded. Clear artifacts were present in the DAS data due to the additional casing surrounding the fiber. However, it is believed that this casing greatly improved the data, as a loss of energy was observed in areas without casing (Daley et al., 2013).

\subsection{Time-lapse seismic}

In an isotropic medium, P-waves are affected, in part, by the fluid compressibility of a porous rock-fluid system. By making time-lapse measurements using P-waves passing through a producing/injecting reservoir, the effects of fluid flow may be monitored on the assumption that the geology is static. This is possible because a change in the compressibility of pore fluid content contributes to a change in the compressibility 
of the rock-fluid system. The greater the change in compressibility between the pore fluids, the greater the time-lapse signal. Additionally, rock matrices with a higher compressibility yield stronger time-lapse signals under fluid replacement than do stiffer matrices, generally speaking (Lumley, 2009).

The term "4D seismic" refers to a time-lapse seismic study where each survey is 3D. Seismic difference volumes can be constructed by subtracting one seismic (or attributes) volume from another produced from a later survey. This requires that both volumes are gridded identically, and that there is adequate repeatability between the two (Huang, 1998). Poor repeatability reduces confidence in the physical meaning of the time-lapse signal, as it becomes difficult to discern noise and processing artifacts from the effects of fluid replacement. Ideally, the signature of time-invariant geology in the 3D seismic data cancels out completely when subtracting one volume from another, leaving only the effects of the fluid flow. 4D seismic data provides the opportunity to image regions outside of the range of well-logging methods, and is thus useful for monitoring fluid migration over large volumes in a reservoir. Lumley $(2001,2009)$ outlines best practices of time-lapse seismic reservoir monitoring, including the use of a normalised root-mean-square (nRMS) to quantify repeatability (Kragh and Christie, 2002).

Modeling seismic data from fluid flow simulations may be used to reinforce interpretations from the time-lapse seismic data. By demonstrating that a signal in a seismic difference volume can be attributed to fluid in a realistic production/injection scenario, fluid-flow simulations build confidence that the identified seismic anomalies are not processing or acquisition artifacts. Constructing realistic injection/production scenarios can be accomplished more accurately after fluid flow has commenced. By 
matching production parameters (injection rate, pressure, temperature, etc.) in the model to those observed in the field, a "history-matched" simulation can be produced. Huang et al. (1998) used seismic data to iteratively refine history-matched reservoir simulations, thus allowing more accurate estimations of saturation distributions over time. Log-based data and a baseline seismic survey were used to develop an initial reservoir model, which was run forward to the time of the monitor survey. The output from the flow simulation was converted to an acoustic model using Gassmann's equations (Gassmann, 1951), and a simple convolutional approach was used for seismic modeling. Differences between the synthetic and field data were minimized using an optimization algorithm.

\subsection{Aquistore seismic monitoring background}

Prior to the baseline surface and VSP surveys at Aquistore, Nader et al. (2012) investigated the effectiveness of seismic methods for monitoring migrating $\mathrm{CO}_{2}$ for the Estevan site. The study used fluid replacement modeling as a means to simulate the migration of $\mathrm{CO}_{2}$ from the injection interval into the overlying saline aquifers to investigate the seismic response to $\mathrm{CO}_{2}$ above the injection interval. Fluid replacement modeling simulates the effects on acoustic properties under a change in pore fluid composition, i.e. brine to $\mathrm{CO}_{2}$. In each of the aquifers, a sharp decrease in acoustic impedance was observed until a $\mathrm{CO}_{2}$ concentration of $10-20 \%$ was reached, then it decreased less rapidly and roughly linearly until a concentration of $70 \%$. This behavior could be attributed primarily to the change in seismic velocity, while the effect of the density change was lesser. The impedance contrasts were more pronounced in the shallower, less saline aquifers, Belly River (668 $\mathrm{m}$ to top) and Mannville (1169 $\mathrm{m}$ to top), 
and less pronounced in the deeper, more saline aquifers, Alida-Tilston (1925 $\mathrm{m}$ to top) and Rupert (2872 $\mathrm{m}$ to top).

The seismic monitoring program at Aquistore includes the use of a permanent sparse areal array of buried geophones. Two pre-injection surveys were undertaken in March 2012 and May 2013 with the objective of characterizing repeatability and overall performance of the 3D array. Both surveys were completed prior to any 3D VSPs at the site. Results from these surveys provided several insights that informed the planning of subsequent monitor surveys. It was demonstrated that buried geophones yielded a 6-7 dB higher SNR than surface deployed geophones, and that dynamite sources yielded a $20 \mathrm{~dB}$ SNR increase over vibroseis (White et al., 2015). Using a standard processing sequence, the reservoir could be imaged, and a global nRMS of 0.07 was achieved after migration (Roach et al., 2015). Data from the first monitor survey (February 2016) were processed in a "4D-friendly simultaneous" workflow (Lumley et al., 2003) with the baseline data, with the objective of investigating injection-related time-lapse anomalies. A high nRMS zone (0.11-0.25) was identified in the reservoir, with amplitudes 2-3 times stronger than the background time-lapse noise (0.05-0.10). Roach and co-workers (2017) attribute the anomaly to an $18 \mathrm{kt}$ plume of $\mathrm{CO}_{2}$ in the upper Deadwood, and concluded that the remaining $18 \mathrm{kt}$ in the reservoir was still below detection threshold.

\subsection{Research objectives}

The research presented in this thesis is organised into three independent studies (corresponding to Chapters 5, 6, and 7 of this integrated thesis), with each marking a contribution to the objective of employing DAS for 3D VSPs to conduct monitoring of the storage site and $\mathrm{CO}_{2}$ plume. The objectives of each study are outlined here: 
1. Establish feasibility of DAS to monitor injected $\mathrm{CO}_{2}$ : The first study in this thesis aims to demonstrate the ability of the DAS system to detect the $\mathrm{CO}_{2}$-based anomalies in the reservoir. This is accomplished by modeling the plume throughout the injection timeline, and comparing its seismic response to an estimate of the time-lapse noise. This study was completed prior to injection, thus the injection parameters in the flow simulations are estimated.

2. Time-lapse 3D VSP imaging of the reservoir: Before time-lapse monitoring may commence, 3D images of the reservoir zone must be obtained. The second study develops a 4D VSP processing flow for the baseline and first monitor data that maximizes repeatability. Time-lapse difference volumes are then computed and interpreted with the goal of detecting a plume-based anomaly in the reservoir.

3. Characterization of the $\mathrm{CO}_{2}$ plume via field and simulation data: The third study employs the results of history-matched fluid flow simulations to forward-model seismic responses. These simulations improve on those used in the first study as they incorporate field measured injection parameters. The plume is characterized by comparing the results of the field surveys with the similarly processed synthetic data. This work also attempts to infer how well the $\mathrm{CO}_{2}$ distribution may be imaged, and what limitations are introduced by imaging conditions.

\subsection{Originality of research}

The first objective of this thesis requires undertaking a time-lapse analysis of DAS data reflecting poorer repeatability conditions than those in Cox et al., (2012). Additionally, the nRMS repeatability images are presented alongside modeled plume responses, thereby contributing a robust demonstration that $\mathrm{CO}_{2}$ can be detected in 
circumstances that better reflect the limitations of long-term time-lapse field studies. The completion of the second objective of this thesis marks the first time that DAS data is used to construct 3D images of actual $\mathrm{CO}_{2}$ in the subsurface. This is an important step in realizing the potential of DAS in CCS applications, as all similar previous studies have been proofs of concept using synthetic data (e.g. Bacci et al., 2017). The third objective builds on the results of the second. By refining the interpretations of the field data with comparisons to modeled data, meaningful characterizations can be made about the size and location of the plume, thus establishing the ability of DAS VSP's to monitor the migration of injected $\mathrm{CO}_{2}$.

When considering the large scale of the surveys and the substantial number of sensors, these datasets are of a size incomparable to any similar previous projects. While a dataset of this size introduces challenges in processing, it also provide exceptional spatial sampling and depth coverage. Challenges involved with attaining adequate repeatability and resolution in the seismic images at this depth require novel techniques in terms of 4D VSP processing and imaging. The objectives outlined above seek to demonstrate that DAS has matured to the extent where these challenges may be overcome, and can be used to monitor $\mathrm{CO}_{2}$ in a deep geological reservoir.

\section{Methods}

\subsection{Overview}

The research presented in this thesis combines VSP processing and imaging techniques with finite-difference (FD) seismic forward modeling to perform 4D 
monitoring of the injected $\mathrm{CO}_{2}$ plume and interpret its size and location throughout the early stages of injection. As the body of this thesis is comprised of three separate manuscripts accepted or submitted in peer-reviewed journals, chapters 5, 6, and 7 contain complete descriptions of the methodologies employed in each study. This chapter aims to present the overarching methodological approach that links each study into a cohesive entity with common objectives.

\subsection{VSP data processing}

The studies presented in chapters 5, 6, and 7 each employ VSP processing sequences designed with the goals of maximizing data repeatability and preparing the data for seismic imaging. In each study, the time-lapse data are processed in "parallel" (Lumley, 2003) in the sense that the multiple vintages are processed asynchronously using effectively identical processing flows. In general, each processing flow aimed to reduce the raw data to deconvolved, upgoing wavefields with a high SNR; the implementations, however, varied from study to study.

The study presented in chapter 5 was undertaken before the acquisition of a monitor survey, and thus there was no knowledge of the specific limitations introduced by using non-identical time-lapse datasets when devising the processing sequence. Consequently, the synthetic time-lapse data in chapter 5 were processed with a comparatively simple sequence. In certain ways, the results of this processing approach produced the best-case scenario for expected time-lapse differences. For example, the sequence did not limit the bandwidth (and hence vertical resolution) to the extent that was required for the field data; nor did the sequence remove near-offset shot gathers as was necessitated by the monitor survey geometry. The processing approach employed on 4D 
field data in chapter 6 built upon the sequence employed in chapter 5, and sought to supress several sources of non-repeatable noise that were absent in the synthetic data. Such sources included variable amplitudes, shifted shot-locations, variable frequency contents and source wavelets. A quality control procedure was introduced in this study to ensure that each operation served to either improve or maintain the level of repeatability achieved by the previous operation. This procedure combined visual inspection with the use of the nRMS repeatability metric.

The processing sequence from chapter 6 was replicated for the synthetic data in chapter 7 to better reproduce processing artifacts and limitations in the seismic images reflecting different injection scenarios, as compared to what was possible in the chapter 5 feasibility study. It is intended that this sequence will be portable to future field and synthetic acquisitions with minimal modifications required for evolving survey geometries and varying file formats.

\subsection{VSP imaging}

Accurate characterization of $\mathrm{CO}_{2}$ plumes in each study required the construction of high-resolution seismic images (see Figure 4-1 for a generalized workflow). VSP imaging techniques adhere to many of the same underlying principles as traditional surface-based methods, but VSP data require special implementations to accommodate its particular acquisition geometry. Such implementations have been understood since the 1980's (Wyatt and Wyatt, 1981; Dillon and Thomson, 1984; Miller et al., 1987), but many commonly available commercial seismic processing suites still do not include VSP imaging capabilities. Consequently, it was of interest to this project to independently develop VSP imaging software. 


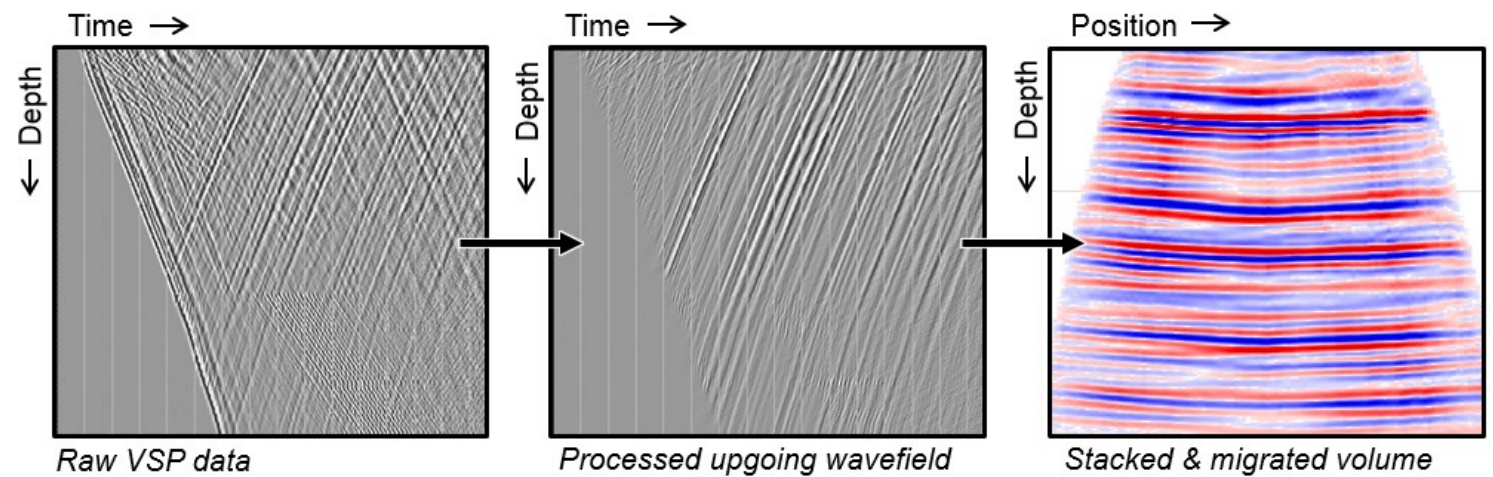

Figure 4-1: Generalized VSP imaging workflow. Raw VSP data (left) are first processed to extract the denoised, deconvolved upgoing wavefield (center). Reflections in the processed data are placed at their subsurface locations via a CDP transform or migration (right).

For the feasibility study presented in chapter 5, synthetic VSP data was generated for two orthogonal shot lines passing through the injection well. Imaging algorithms in 2D were developed to construct seismic images corresponding to each shot line. The first algorithm was a common depth-point (CDP) transform based on the formulations of Dillon and Thomson (1984). This algorithm is a geometric transformation that involves stretching trace data over a reflection curve that is determined via raytracing. The CDP transform maps only specular energy and is equivalent to migration with a $0^{\circ}$ aperture. As such, this algorithm was able to construct images of the reservoir and accurately position reflectors, but was unable to image locations that did not contain a specular reflection. With the absence of a zero-offset shot, the zone beneath the observation and injection wells could not be imaged with the CDP transform. This issue necessitated the development of the 2D Kirchhoff migration algorithm described in section 5.3.2. All migration methods involve the back propagation of the seismic wavefield (as opposed to a simple ray approximation) from the point of measurement to the reflector. Kirchhoff 
migration uses the integral form (Kirchhoff equation) of the wave equation to represent the wavefield at any given point. The implementation of this method requires stacking the weighted trace data along curves defined by the travel-time of energy scattered by image points in the subsurface. In this way, diffractions are collapsed to their respective sources (Yilmaz, 1987). This treatment of diffracted energy allows imaging of locations not illuminated by specular reflections, in this case, for example, locations beneath the observation and injection wells. Kirchhoff migration was chosen for the simplicity of its implementation and relatively low computational costs. More sophisticated methods (e.g. reverse time migration) are better able to image complex structure, but are computationally intensive, and likely not required for the flat stratigraphy at the Aquistore site.

The 2D Kirchhoff migration software used in the feasibility study in chapter 5 was written in $\mathrm{C}$, and input/output was controlled with a MATLAB script. The study demonstrated that Kirchhoff migration was an appropriate technique for accurately imaging the $\mathrm{CO}_{2}$ plume. A commercial 3D Kirchhoff migration algorithm (VSProwess) was used for the 3D imaging presented in chapters 6 and 7.

\subsection{Fluid-replacement modeling}

The research presented in chapters 5 and 7 aims to model the seismic responses from several injections scenarios provided by the Energy and Environmental Research Center (EERC). This requires an elastic model of the subsurface that incorporates the effects of $\mathrm{CO}_{2}$ replacing brine in the pore-space of the reservoir rocks. This is accomplished via a process termed fluid-replacement modeling (or fluid-substitution modeling). This section provides a concise overview of the method employed in both 
studies, as formulated in Gassmann (1951) and outlined in Smith et al. (2003). A description of the parameters and assumptions used in the fluid-replacement modeling procedures undertaken in this research is presented in sections 5.4.3 and 7.3.2.

P- and S-wave velocities depend on bulk modulus $K$, shear modulus $\mu$, and density $\rho$ as follows:

$$
\begin{aligned}
& v_{p}=\sqrt{\frac{K+\frac{4}{3} \mu}{\rho}} \\
& v_{S}=\sqrt{\frac{\mu}{\rho}}
\end{aligned}
$$

The shear modulus of a rock remains unchanged throughout fluid-substitution, but bulk modulus and density do not. The Gassmann equation is used to compute the new bulk modulus $K_{\text {sat }}$ by relating it to porosity $\varphi$, bulk modulus of the porous rock frame $K^{*}$, bulk modulus of the mineral matrix $K_{o}$, and bulk modulus of the pore fluids $K_{f l}$ :

$$
K_{s a t}=K^{*}+\frac{\left(1-\frac{K^{*}}{K_{o}}\right)^{2}}{\frac{\varphi}{K_{f l}}+\frac{(1-\varphi)}{K_{o}}-\frac{K^{*}}{K_{o}^{2}}}
$$

Calculating $\mathrm{K}_{\text {sat }}$ first requires the computation of the bulk modulus of the porous rock frame (unsaturated rock). This is done by rearranging equation 2 to solve for $K^{*}$ using the bulk modulus of the in-situ fluids for $K_{f l}$. For this calculation, the in-situ $K_{\text {sat }}$ may be calculated using log-based velocity and density values:

$$
K_{s a t}=\rho\left(v_{p}^{2}-\frac{4}{3} v_{s}^{2}\right)
$$


$K_{o}$ may be calculated similarly using lab-measured mineral grain velocities and densities in place of the in situ measured values. After $K^{*}$ is calculated, the only remaining parameter required to compute the new $K_{\text {sat }}$ is the bulk modulus of the new fluid mixture. For a simple two-fluid system, this can be determined as follows:

$$
K_{f l}=\left[\frac{S_{1}}{K_{1}}+\frac{\left(1-S_{1}\right)}{K_{2}}\right]^{-1}
$$

Where $K_{l}$ and $K_{2}$ are the bulk moduli of the two fluids, and $S_{l}$ is the saturation of the first fluid (the saturation of the second fluid $\mathrm{S}_{2}$ is related to $\mathrm{S}_{1}$ by $S_{2}=1-S_{1}$ ). Similarly, the density of the two-fluid system can be determined as:

$$
\rho_{f l}=S_{1} \rho_{1}+\left(1-S_{1}\right) \rho_{2}
$$

Where $\rho_{1}$ and $\rho_{2}$ are the densities of the two fluids. Finally, the density of the new saturated rock can be calculated as well:

$$
\rho=\varphi \rho_{f l}+(1-\varphi) \rho_{g}
$$

Where $\rho_{g}$ is the lab-measured mineral grain density. These new bulk moduli and densities may now be used to compute the new seismic velocities for the elastic model using equations $1 \mathrm{a}$ and $1 \mathrm{~b}$.

\subsection{Seismic modeling with finite differences}

Analytical solutions to the wave equation do not exist for most survey and subsurface geometries, and therefore must be estimated by numerical methods. Due to recent advances in supercomputing, elastic 3D finite difference modeling has become a viable method to generate synthetic seismic data. While an in-depth description of the 
finite-difference method employed in this research is beyond the scope of this thesis, it follows the formulations overviewed by Bohlen (2002).

In the feasibility study presented in chapter 5 , two orthogonal lines of shots were simulated to determine the seismic response of the $\mathrm{CO}_{2}$ for multiple injection scenarios. At this stage of the research, the remainder of the shot grid was not simulated due to the fact that many injection scenarios were investigated for several $\mathrm{CO}_{2}$ volumes, and each required considerable runtime to forward model. After injection had commenced in 2015, the history-matched flow simulations were used to construct the elastic models described in chapter 7, and each of the 50 shots analyzed in the chapter 6 study were incorporated in the seismic simulations. As this model more closely replicated field shot geometry and injected $\mathrm{CO}_{2}$ volumes, greater effort was put forth to model the receiver array. This required reducing the grid spacing along the depth axis from $5 \mathrm{~m}$ to $2 \mathrm{~m}$ in order to produce a trace for each DAS channel without interpolating. This regridding resulted in the runtime for each shot increasing from 1 hour to 4 hours. Specific parameters and considerations relevant to the seismic modeling performed in this research can be found in sections 5.4.3 and 7.3.2. 


\section{Feasibility of Time-lapse VSP Monitoring at the Aquistore $\mathrm{CO}_{2}$ Storage Site Using a Distributed Acoustic \\ Sensing System}

\subsection{Abstract}

The Aquistore carbon storage project, located near Estevan, Saskatchewan, Canada, aims to employ 3D time-lapse seismic techniques to monitor injected $\mathrm{CO}_{2}$ at depths of 3100 - $3350 \mathrm{~m}$. During early stages of the injection schedule, vertical seismic profiling (VSP) will primarily be utilized, given its inherent advantages in imaging close to the borehole. Distributed acoustic sensing (DAS) possesses the capability of providing a cost-efficient, high-resolution alternative to traditional geophone methods in VSP. In this study, an evaluation is made of baseline DAS and traditional geophone VSP data from an observation well located $150 \mathrm{~m}$ away from the injection well. Comparative images are analyzed for quantities of injected $\mathrm{CO}_{2}$, ranging from $27 \mathrm{kt}$ to $330 \mathrm{kt}$ to determine the visibility of the $\mathrm{CO}_{2}$ plume over time. The study demonstrated that DAS VSP is a feasible technique for reservoir monitoring at the Aquistore site. The $\mathrm{CO}_{2}$ plume should be visible near the borehole after 90 days $\left(27 \mathrm{kt}\right.$ of $\left.\mathrm{CO}_{2}\right)$ of injection, with increasing clarity over a three-year duration.

\subsection{Introduction}

Aquistore is a geological carbon storage project, where $\mathrm{CO}_{2}$ is captured from a nearby coal-fired power plant (SaskPower's Boundary Dam Power Station), transported via pipeline to the Aquistore site, and injected for long-term storage into a deep ( 3200 
m) sandstone formation in the Williston Basin (see Figure 5-1 for location). Monitoring of the $\mathrm{CO}_{2}$ plume is necessary to ensure its containment within the reservoir. Both surface seismic (e.g., Arts et al., 2004; Urosevic et al., 2010; White, 2013; Ivandic et al., 2015) and vertical seismic profiles (VSP; e.g., Ahmadi and Morozov, 2011; Coueslan et al., 2013) are commonly employed for monitoring in carbon storage projects. VSP monitoring is often preferred during the initial stages of injection due to the capability of VSP to image near the borehole with higher frequency content and greater signal to noise ratio (SNR) when compared to an equivalent surface seismic survey (O'Brien et al., 2004). Other advantages of VSP include the ability to accurately tie reflections to geological horizons intersected by the wellbore, reliably verify velocity models with a zero-offset shot, characterize downgoing energy, and reduce ghosting effects (Hardage, 2000; Kuzmiski et al., 2009). At Aquistore, VSP is currently being utilized for time-lapse monitoring during the early stages of injection.

The past fifteen years has seen the emergence of optical fiber-based seismic sensors employed in seismic surveying (Bostick, 2011), particularly in geophysical surveillance and in-well monitoring. The Aquistore project has utilized distributed acoustic sensing (DAS) for VSP employing a fiber optic cable that is permanently deployed on the outside of the casing of a deep observation well.

This study aims to investigate the feasibility of using a DAS system for time-lapse VSP monitoring of the Aquistore $\mathrm{CO}_{2}$ storage. First, we compare DAS VSP results against data recorded simultaneously with a conventional wireline geophone system. The real DAS data are then used to estimate the degree of data repeatability that can be achieved with this system. In the second part of the study, synthetic time-lapse VSP data 
are calculated and processed for $\mathrm{CO}_{2}$ fluid flow simulations that have been done for $\mathrm{CO}_{2}$ injection quantities ranging from $27 \mathrm{kt}$ to $330 \mathrm{kt}$. The resultant synthetic time-lapse VSP images are compared against the data repeatability from the real data to assess the expected visibility of the $\mathrm{CO}_{2}$ plume over time and to evaluate the feasibility of long-term monitoring of the $\mathrm{CO}_{2}$ plume at the Aquistore site.

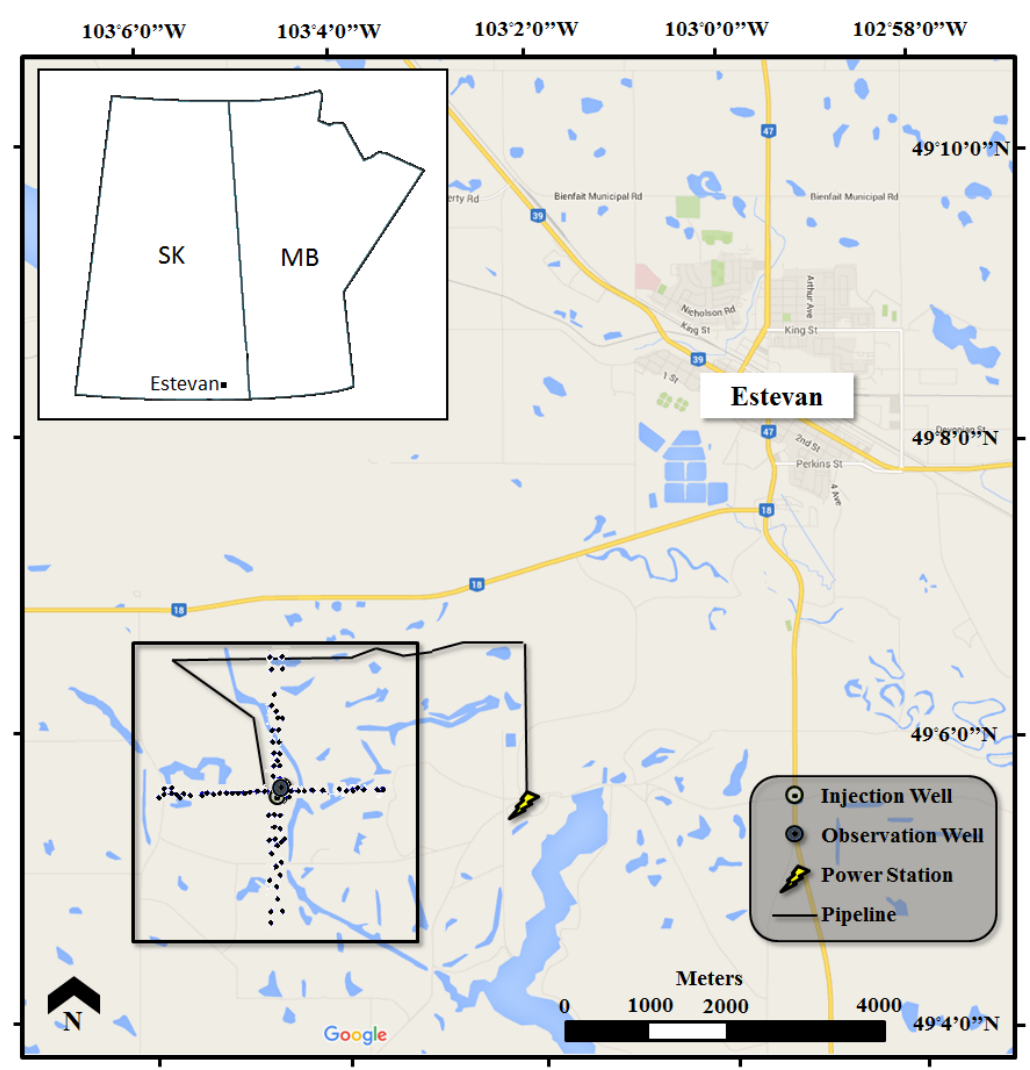

Figure 5-1: Location map of the Aquistore $\mathrm{CO}_{2}$ storage site (boxed area). The east-west and north-south lines selected for image comparisons are indicated as black dots.

\subsubsection{Geological setting}

The Williston Basin is ideal for $\mathrm{CO}_{2}$ storage and has already been employed for such in previous projects. The basin contains an abundance of depleted petroleum reservoirs which have successfully hosted injected $\mathrm{CO}_{2}$ at various depths in the Weyburn- 
Midale (Saskatchewan, Canada) and Northwest McGregor (North Dakota, United States) projects. Coal seams in the basin have also been shown to retain injected $\mathrm{CO}_{2}$ in the Lignite Field Validation Test in Burke County (North Dakota, United States).

At the Aquistore site, the basin is approximately $3300 \mathrm{~m}$ thick and is made up of Paleozoic carbonates and evaporites, and Mesozoic sandstones and shales. The Winnipeg and Deadwood formations reside at the bottom of the stratigraphic column sitting unconformably on the Precambrian basement. Brine-filled porous sandstones of the Winnipeg and Deadwood formations constitute the target reservoir for $\mathrm{CO}_{2}$ injection and storage. Together, these formations are approximately $150 \mathrm{~m}$ thick and are capped by the Icebox shale, which as the uppermost unit of the Winnipeg formation forms the principal local sealing unit. The Prairie Evaporite in this region is $\sim 150 \mathrm{~m}$ thick and occurs at a depth of $\sim 2500 \mathrm{~m}$. It forms the primary regional seal for deep $\mathrm{CO}_{2}$ storage. Regionally, the target reservoir formations are characterized by high permeabilities of up to $1000 \mathrm{mD}$ and moderate porosities of $\sim 11-17 \%$ (Whittaker and Worth, 2011), whereas the Aquistore site is characterized by somewhat lower porosities and permeabilities (see Table 5-1). 
Table 5-1: Petrophysical properties of reservoir units at Aquistore based on injection well log measurements made in 2013 (EERC, 2014).

\begin{tabular}{ccccccc}
\hline Name & $\begin{array}{c}\text { Top } \\
\text { depth } \\
(\mathbf{m})\end{array}$ & $\begin{array}{c}\text { Median } \\
\text { Vp }(\mathbf{m} / \mathbf{s})\end{array}$ & $\begin{array}{c}\text { Median } \\
\text { Vs }(\mathbf{m} / \mathbf{s})\end{array}$ & $\begin{array}{c}\text { Median } \\
\text { density } \\
\left(\mathbf{k g} / \mathbf{m}^{3}\right)\end{array}$ & $\begin{array}{c}\text { Median } \\
\text { Effective } \\
\text { Porosity } \\
\text { (fractional) }\end{array}$ & $\begin{array}{c}\text { Median } \\
\text { Permeability } \\
\text { (mD) }\end{array}$ \\
\hline \hline $\begin{array}{c}\text { Upper Black Island } \\
\text { (sandstone) }\end{array}$ & 3155 & 4933 & 3090 & 2574 & 0.04 & 1.7 \\
\hline $\begin{array}{c}\text { Lower Black Island } \\
\text { (sandstone) }\end{array}$ & 3170 & 4868 & 2992 & 2542 & 0.049 & 2.5 \\
\hline $\begin{array}{c}\text { Deadwood D } \\
\text { (sandstone) }\end{array}$ & 3231 & 4868 & 2989 & 2488 & 0.073 & 23.8 \\
\hline $\begin{array}{c}\text { Deadwood C } \\
\text { (sandstone) }\end{array}$ & 3257 & 4639 & 2774 & 2580 & 0.047 & 10.2 \\
\hline $\begin{array}{l}\text { Deadwood B } \\
\text { (sandstone) }\end{array}$ & 3281 & 5258 & 2891 & 2746 & 0.033 \\
\hline $\begin{array}{l}\text { Deadwood A } \\
\text { (sandstone) }\end{array}$ & 3297 & 4897 & 3043 & 2540 & 0.05 & 6.0 \\
\hline
\end{tabular}

\subsubsection{Distributed Acoustic Sensing (DAS)}

DAS technology eliminates the need for discrete point sensors by using the fiber itself as the sensor (Mestayer et al., 2011; Miller et al., 2012). This allows the fiber to be employed as a continuous array of receivers. DAS does not require manufactured sensors as is the case in discrete sensor systems, and only a single fiber is needed (Bostick, 2000; Hornby and Burch, 2008).

In DAS, incoming seismic waves impart localized strain on the fiber, altering the backscatter of light pulses within. This requires that the fiber is coupled via friction or pressure with the surrounding medium through which the seismic wave is propagating. The amplitude and timing of the detected variation in optical backscatter is used to 
compute estimates of localized strain rates in the fiber, and can thus be utilized as a distributed acoustic array capable of recording thousands of channels (Shatalin et al., 1998; Mestayer et al., 2011; Miller et al., 2012). Strain rate measurements are taken by interrogating a length of the fiber (a predetermined 'gauge length') centered on a given sensor location. Sensor spacing and gauge length need not be equal, and a measurement from a given sensor corresponds to an average across the gauge length. Nearly any optical cable may be used for DAS and can remain installed down-hole for years (Mestayer et al., 2011). With permanently installed fibers, DAS offers a reduced cost alternative to traditional geophone methods, as each subsequent survey does not require any further downhole deployment, whereas repeated temporary deployment of geophones via wireline can be very costly. Also, the depth extent of the monitoring interval for DAS is generally greater than what is practical for borehole geophone wirelines. This is especially advantageous for geological storage monitoring (as compared to monitoring oil reservoirs) where a larger depth extent is required to track possible $\mathrm{CO}_{2}$ migration into overlying units. Repeatability is also improved with the fiber permanently deployed, as wireline geophones are subject to coupling consistency issues; i.e., the repeatability of the signal depends on how well the clamping of the geophone tool can be reproduced. Also, there is some risk of tool loss and/or wellbore compromise each time the wireline is deployed. Currently, DAS systems are limited to high sensitivity only along the longitudinal axis (Hornman, 2016). Thus a vertically oriented fiber essentially measures only vertical components of the wavefields. 


\subsection{Methods}

\subsubsection{Data acquisition}

The observation well at the Aquistore site, drilled and completed during the fall of 2012, has been used to acquire VSP data. Stainless steel tubing with a diameter of 6.35 $\mathrm{mm}(0.25$ inch $)$ containing a bundle of optical fibers ( 2 multimode and 2 single mode $)$ was strapped to the casing and cemented in place during well completion. At the end of deployment, $2766 \mathrm{~m}$ of the total $3400 \mathrm{~m}$ of fiber installed was found to be operational. The optical fibers in the well provide continuous temperature monitoring (multimode fibers) and DAS recording capability.

The success of an initial test of DAS recording at the Aquistore site in May, 2013 (Daley et al., 2014) led to the execution of an expanded pre-injection baseline 3D VSP in November 2013. This survey was recorded with both a downhole geophone array and with DAS systems connected to single-mode and multimode fibers in the observation well, allowing direct comparisons to be made between the 3 VSP data sets. The singlemode fiber DAS data are used for the comparisons with geophone data in this study. Subsequent monitoring surveys are planned with the schedule dependent on the $\mathrm{CO}_{2}$ injection schedule.

A total of 670 source locations were occupied for the 3D VSP survey. A semiregular 3000 x $3000 \mathrm{~m}$ grid was used with nominal in-line and inter-line spacings of $\sim 72$ $\mathrm{m}$ and $\sim 144 \mathrm{~m}$, respectively. The original shot grid was designed based on 924 shot points, a minimum of 30-fold coverage at the reservoir depth for a 60 -level geophone array deployed from $1650 \mathrm{~m}$ to $2650 \mathrm{~m}$ depth, and assuming bin dimensions of $36 \mathrm{x} 36 \mathrm{~m}$ 
for processing. The regular grid was modified to accommodate inaccessible sites at the surface. Also, the total number of shots was reduced (to reduce costs) by eliminating shots and increasing the shot spacing at the larger offsets from the observation well. Sources consisted of single $1 \mathrm{~kg}$ dynamite charges located at a depth of $15 \mathrm{~m}$.

The wireline geophone array consisted of 57 three-component (analogue electromechanical sensors) geophones installed with a vertical spacing of $15 \mathrm{~m}$, centered at a depth of $1900 \mathrm{~m}$. The geophones measure particle velocity, and were installed in a Sercel Maxiwave tool, which was clamped in the observation well. The geophones have an output of $0.402 \mathrm{~V} / \mathrm{cm} / \mathrm{s}$ at $0.47 \%$ damping, and a resonant frequency of $15 \mathrm{~Hz}$. The DAS data were acquired with a Silixa iDAS system using a $10 \mathrm{~m}$ gauge length, and preprocessing of the raw optical data to produce seismic data was done by Silixa LTD. The raw optical data which are a measure of strain rate were processed to emulate a seismic sensor spacing of $2 \mathrm{~m}$ resulting in 1383 traces per shot over the 2766 depth interval (Harris et al., 2015). This response can be converted to particle velocity by first integrating the traces in the time domain to convert strain rate to strain. This step is termed rebalancing since it effectively boosts the lower frequencies. Scaling this strain value by the propagation speed along the fiber, or apparent velocity, converts the response to particle velocity, as explained in Daley et al. (2015).

\subsubsection{Data processing}

Each shot gather was processed using similar processing flows for both traditional geophones and DAS data. In this way controlled comparisons could be made throughout the analysis. Generally, the processing flow consists of the following: spherical divergence correction, spiking deconvolution, notch and low-pass filtering, and $f-k$ 
filtering to remove the downgoing wavefield. A 2D Kirchhoff migration program employing the formulation of Schneider (1978) and Dillon (1988) was written to create 2D images of the subsurface at specified azimuths. A 1D isotropic velocity model was utilized in this process, and was based on sonic log measurements from the observation well, and first arrivals in a zero-offset shot. Travel time computations were performed with a 3D eikonal solver (Hole and Zelt, 1995). Multiple gathers were then stacked to build coherent images using spatial bins of dimensions $10 \times 10 \mathrm{~m}$ in the horizontal and vertical dimensions, respectively. A dip-limited migration aperture was maintained at $6^{\circ}$ due to the flatness of the stratigraphy. Prior to migrating the DAS data the response was converted to particle velocity so that the final image was geophone equivalent. 


\subsection{Seismic results}

\subsubsection{Traditional geophones versus DAS}

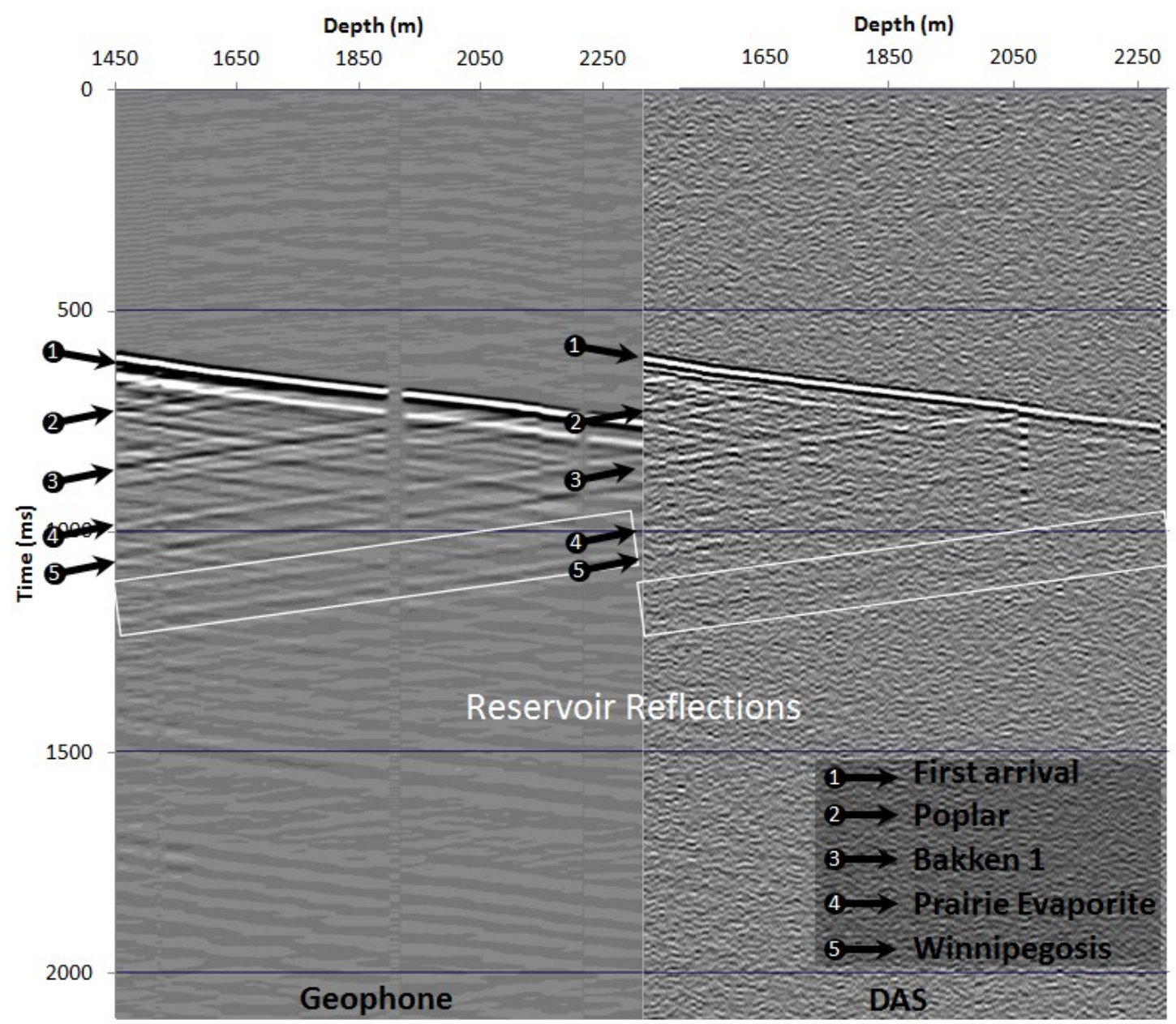

Figure 5-2: Comparison of raw vertical-component geophone data (left) and single-mode DAS data (right) for a single shot gather in the depth range of $1455-2295 \mathrm{~m}$, where the receiver lines overlap. Of the 57 geophones, 3 did not record and yielded dead traces, resulting in 54 geophone traces and 963 DAS traces over this interval. The corresponding shot point is 46083740 , which is located at a distance of $470 \mathrm{~m}$ from the observation well. 


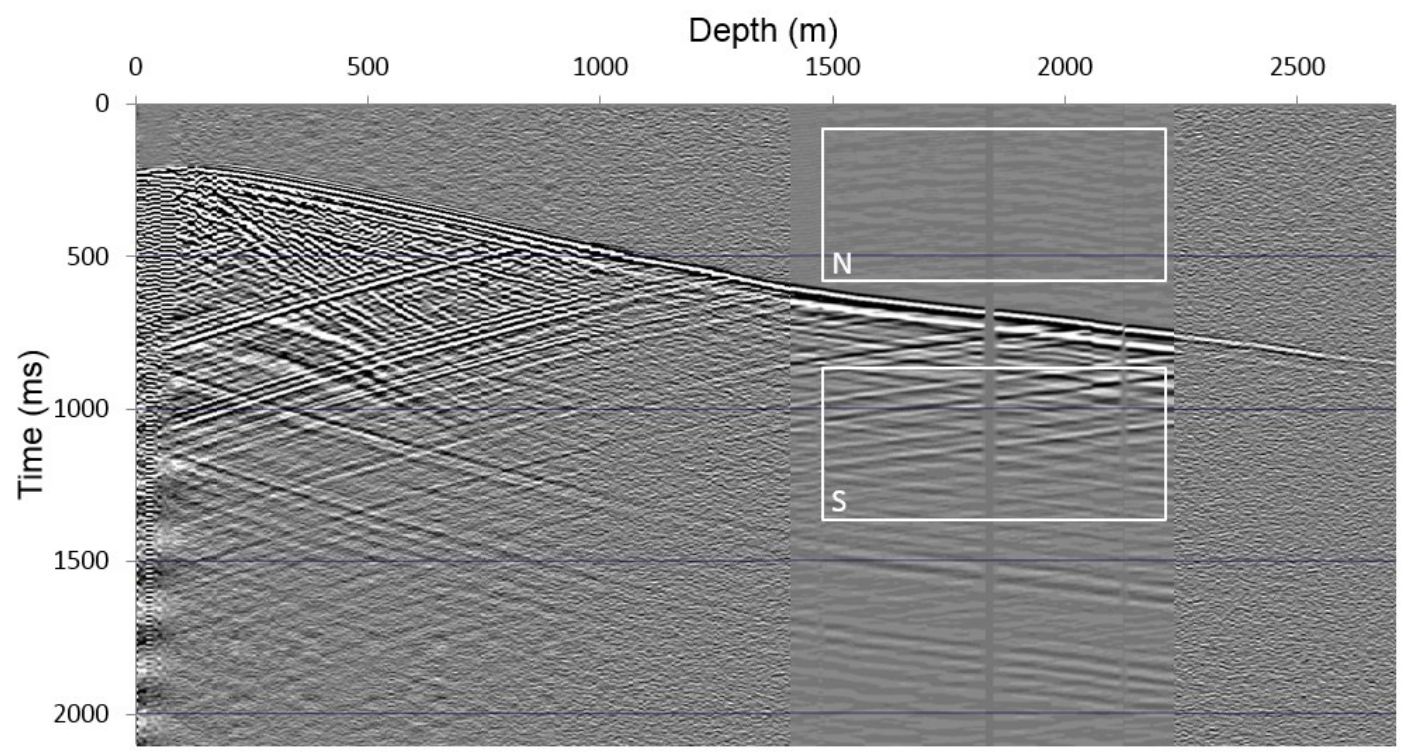

Figure 5-3: Comparison of raw DAS seismic data and vertical-component geophone data for a single shot gather. The corresponding shot point is 46083740 which is located at a distance of $470 \mathrm{~m}$ from the observation well. The geophone traces replace the DAS data in the depth range of $1455-2295 \mathrm{~m}$. There are 963 single-mode DAS traces spaced at $2 \mathrm{~m}$ intervals and 57 vertical-component geophone traces spaced at $15 \mathrm{~m}$ intervals in the plot. Of the 57 geophones, 3 did not record and yielded dead traces. $S$ and $N$ rectangles show the signal and noise windows, respectively, used for Figure 5-4 (figure modified from Harris, 2015).

Raw wireline geophone data and raw DAS data are compared in Figure 5-2 and Figure 5-3. A direct comparison over the same depth interval (Figure 5-2) highlights the direct arrival and various reflections. The reservoir reflections, found within the white box, are faintly above the noise in the DAS section. In Figure 5-3, the reflections in the DAS data generally decay in amplitude as depth increases, and have visibly lower signal than the geophones at equivalent depth. One of the factors contributing to this pertains to the directivity of the DAS system. The fibers are primarily sensitive to longitudinal strain (vertical in this case), and deeper sensors record P-waves approaching at larger angles to 
the vertical than do their shallower counterparts (Parker et al., 2014). Also visible in Figure 5-3 is the abrupt decrease in signal strength at $\sim 1000 \mathrm{~m}$, which is the result of a known degradation of cement quality resulting in poorer coupling. There is also an impedance contrast between zones of different cement quality that results in guided waves and further signal loss

The frequency responses of geophones and DAS are shown in Figure 5-4. The geophone signal-to-noise-ratio (SNR) is up to $45 \mathrm{~dB}$ whereas a maximum SNR of $15 \mathrm{~dB}$ is observed for the DAS data. Also, the bandwidth where SNR $>1$ is larger for the geophone data $(\sim 5-150 \mathrm{~Hz})$ as compared to the DAS data $(\sim 0-80 \mathrm{~Hz})$. In the case of the DAS data, the higher spatial sampling (relative to that of the geophones) can be utilized to improve SNR by multi-trace data summation methods (i.e., "stacking"), but at the expense of lowering spatial sampling. The wider bandwidth of the geophone data implies that higher resolution should be achievable with this method. 

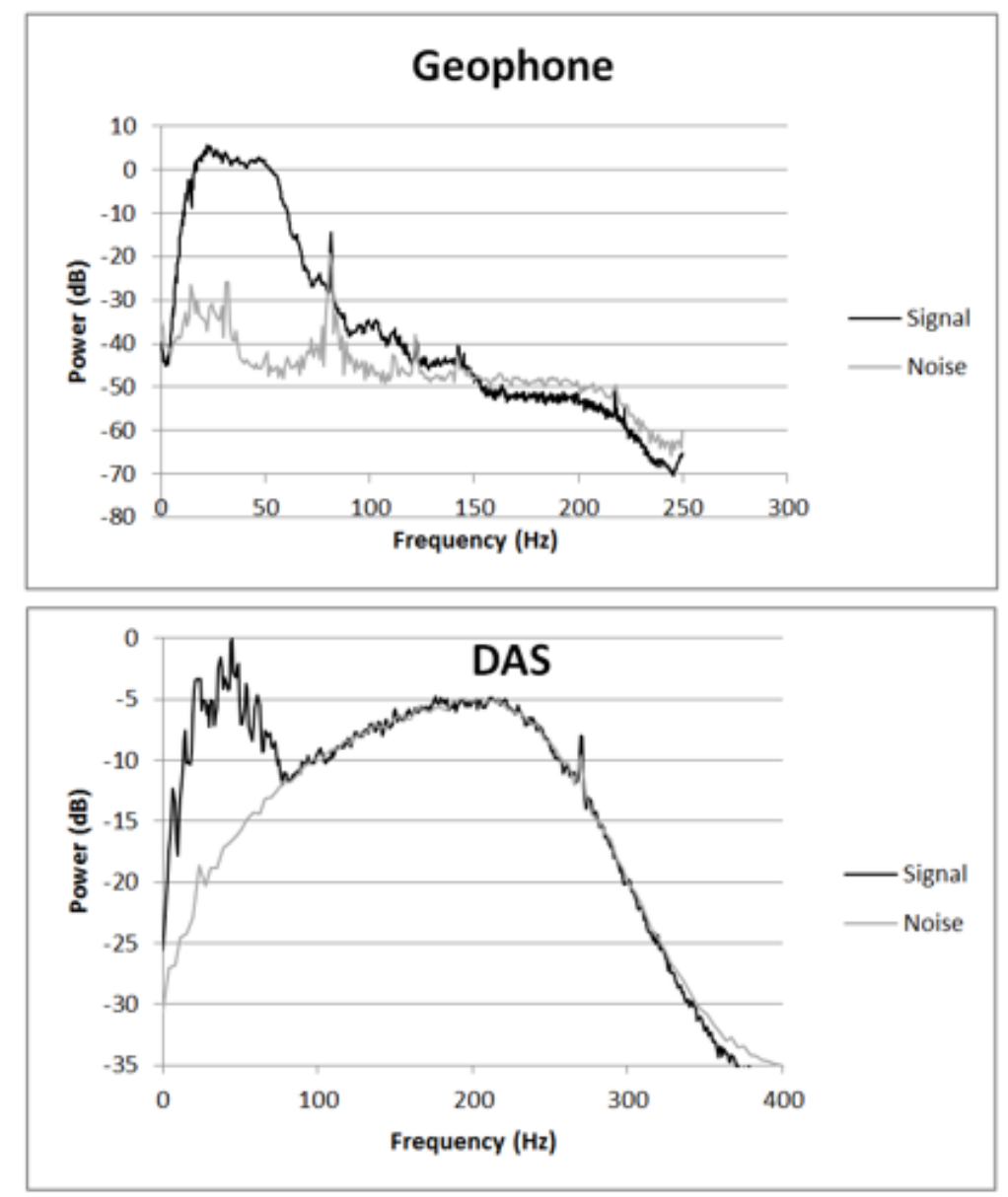

Figure 5-4: Signal and noise power spectra for geophone data (top) and DAS (bottom). As shown in Figure 5-3, the data windows for the noise calculations were chosen to exclude downgoing primary energy.

Figure 5-5 shows a comparison of VSP images for a 2D east-west section produced from the single-mode DAS data and the vertical-component geophone data. These VSP images are superposed on a comparable section modified from the 3D seismic volume of Roach et al. (2014) which was computed using data from a surface-based seismic survey. The DAS and geophone images were computed using the migration program previously described. Upgoing wavefields from 8 shot gathers located west of the observation well were used. The reflections in the VSP images are well aligned with those of the surface- 
based image, with maximum deviations of $40 \mathrm{~m}$ in the deeper reflections, an acceptable difference since the 3D seismic volume was converted to depth using an independent velocity model. Though geological boundaries are not as well defined in these VSP images compared to the surface-based seismic image, clarity is expected to improve with more sophisticated processing and the inclusion of more shots in the stacking process during 3D imaging.

Two characteristics of the DAS versus geophone images in Figure 5-5 are noteworthy. The DAS image provides more extensive spatial imaging above the reservoir as well as a smaller "blind spot" at depths exceeding the recording depth. These effects are the result of the larger depth interval over which the DAS records the seismic wavefield as compared to the geophone array. Comparable depth coverage could be achieved by the deployment of geophones over an equivalent depth interval but is somewhat impractical. 


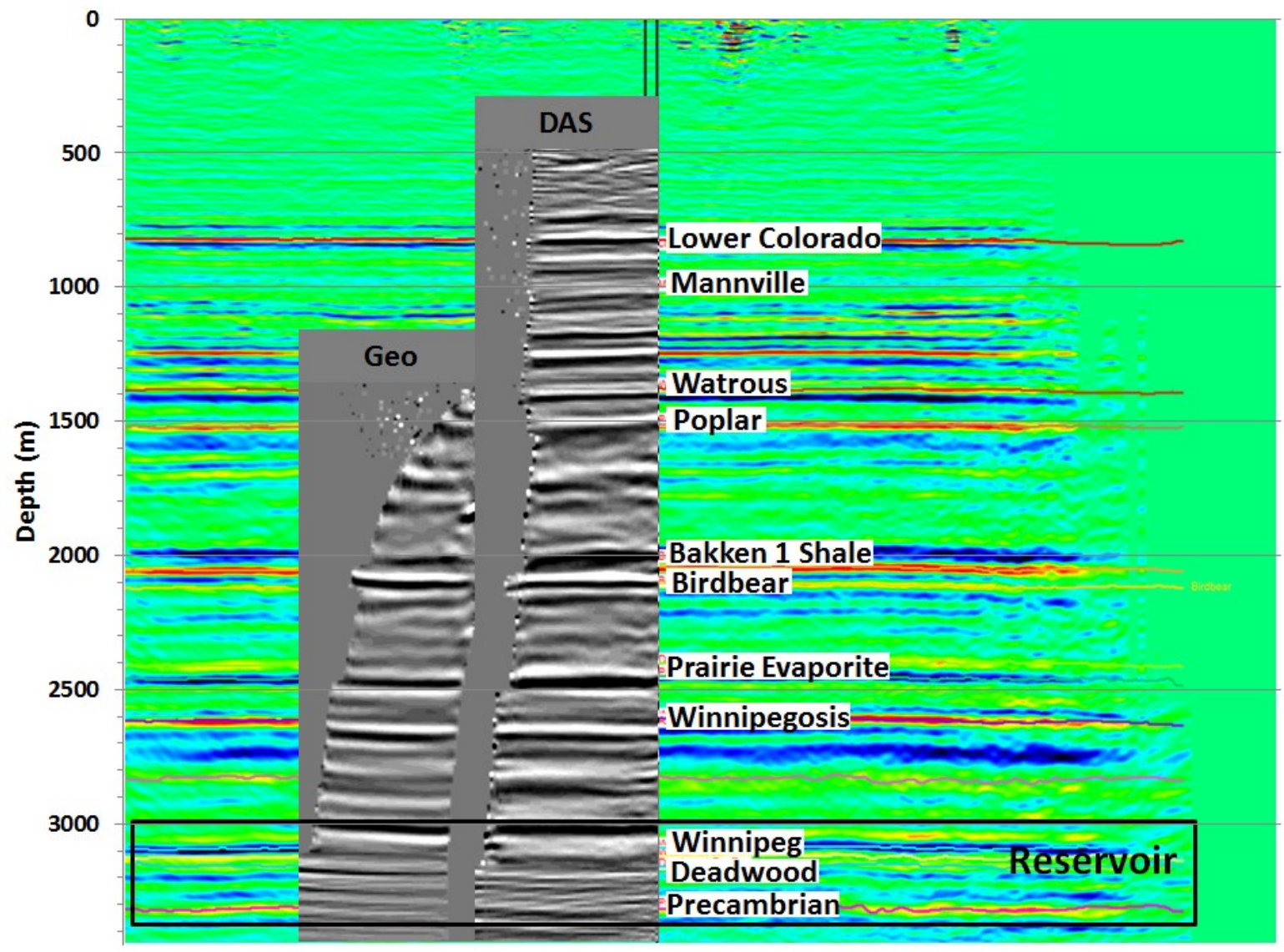

Figure 5-5: 8-fold migrated images of the single-mode DAS and vertical-component geophone VSP data (grey) with migrated section of surface data (green/blue/red) along the east-west line shown in Figure 5-1. Locations of various geological formations are labelled (modified from Harris et al., 2015). Geophone image shows same area as the DAS image, but has been shifted west for comparison purposes. Time-variant spectral whitening was applied to the surface section after time-migration (White et al., 2016), but not to the VSP. This results in the surface data appearing to have a higher dominant frequency, particularly in the deeper reflections. 


\subsubsection{Seismic repeatability}

Time-lapse monitoring is fundamentally based on the repeatability of the imaging process from survey to survey. The non-repeatability of the data (in acquisition and processing) provides a lower limit on the injection-induced geological changes that can be detected. Estimates of data repeatability are most easily obtained by comparison of repeat surveys (e.g., White et al. 2015). In the absence of equivalent data from repeat VSP surveys, in this study we utilize two shots from the November 2013 pre-injection baseline survey that are located within $10 \mathrm{~m}$ of each other and $\sim 1000 \mathrm{~m}$ from the observation well where the wavefield was recorded. The premise for this comparison is that shot points that are located very close to each other relative to the shot-receiver offset should yield very similar although not identical data. The data from the two shots underwent a trace-by-trace cross-equalization process before a direct comparison was made. This included the application of time and phase shifts, frequency spectrum shaping filters, and amplitude scaling factors derived from RMS measurements. Each process utilized reference windows taken well above the reservoir zone. Data differences are measured using a normalized RMS (or nRMS) amplitude difference defined as:

$$
n R M S=\frac{2 \times R M S_{2-1}}{R M S_{1}+R M S_{2}}
$$

This quantity ranges between 0 and 2, corresponding to identical and reversed polarity data, respectively (Kragh and Christie, 2002). A map of nRMS values was computed using a $50 \mathrm{~m}$ sliding window and was considered to represent a lower bound on the repeatability of the DAS surveys. 
Figure 5-6 shows the two migrated shot gathers used for this comparison as well as their amplitude differences before and after cross-equalization. The cross-equalization was successful in aligning reflections and reconciling amplitudes throughout the illuminated area, albeit most drastically above the reservoir.

The nRMS of the amplitude difference calculated for these data is shown in Figure 5-7A. Most of the nRMS values are below 0.5 , although significantly higher values are seen near the edges of the illuminated area and at $2550 \mathrm{~m}$. The edges are expected to exhibit higher nRMS values as these locations correspond to very low amplitudes as visible in Figure 5-6. The RMS of the differences here becomes exaggerated when normalized by these amplitudes. A histogram of the nRMS values is shown in Figure 5-7B, where the mean nRMS is 0.52 , and the mode is 0.29 . The mean has been skewed by the high nRMS values near the edges and thus the mode may be taken as a more representative reservoir value. There does not appear to be any significant change in repeatability with respect to depth until below the reservoir. 

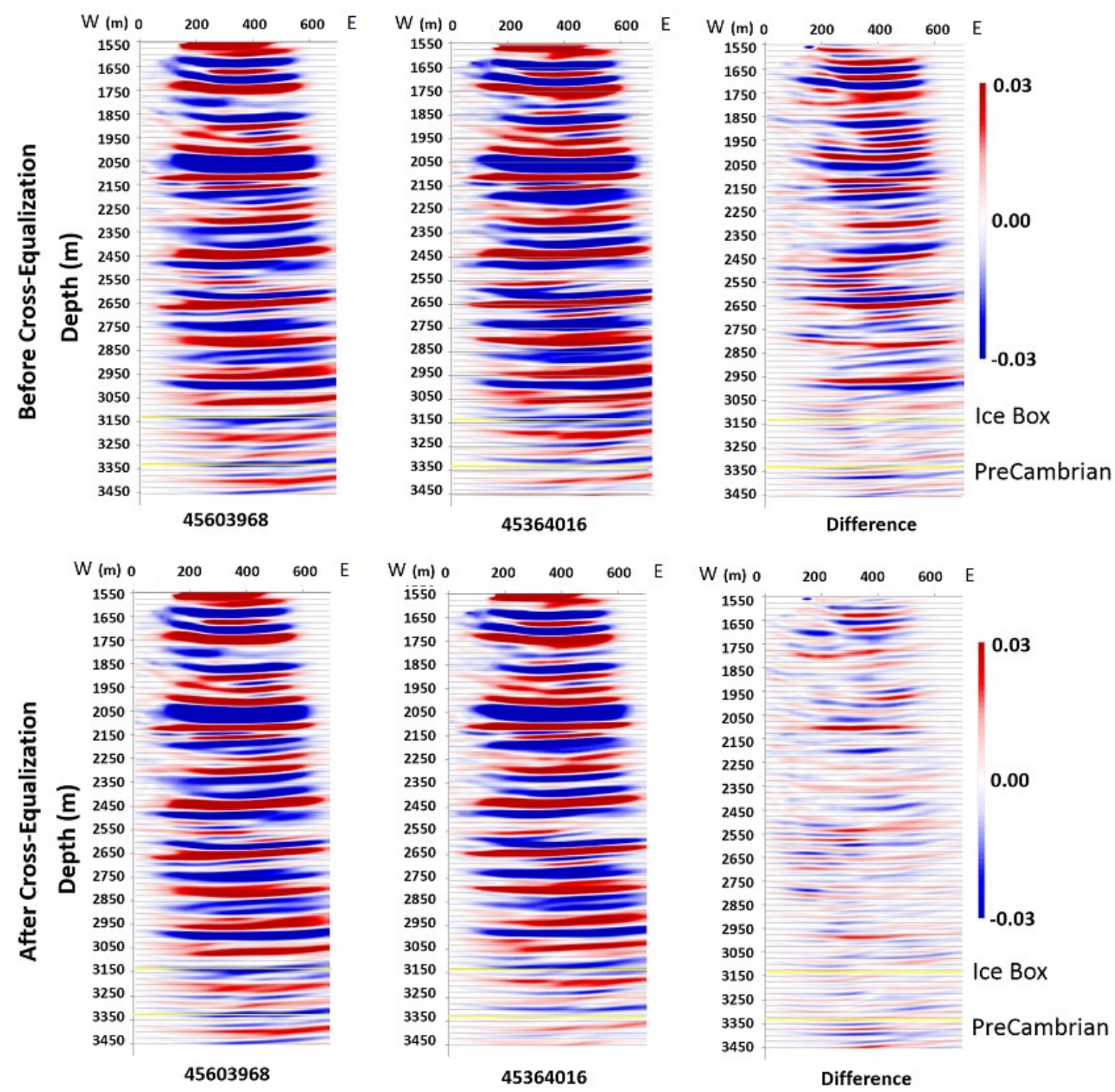

Figure 5-6: 1000 m offset shots from the November 2013 pre-injection baseline survey used for repeatability analysis. Shot 45364016 (middle) underwent trace-by-trace crossequalization to align horizons and match amplitudes to shot 45603968 (left). Resulting amplitude difference images are shown on the right. Observation well is located at $0 \mathrm{~m}$. 


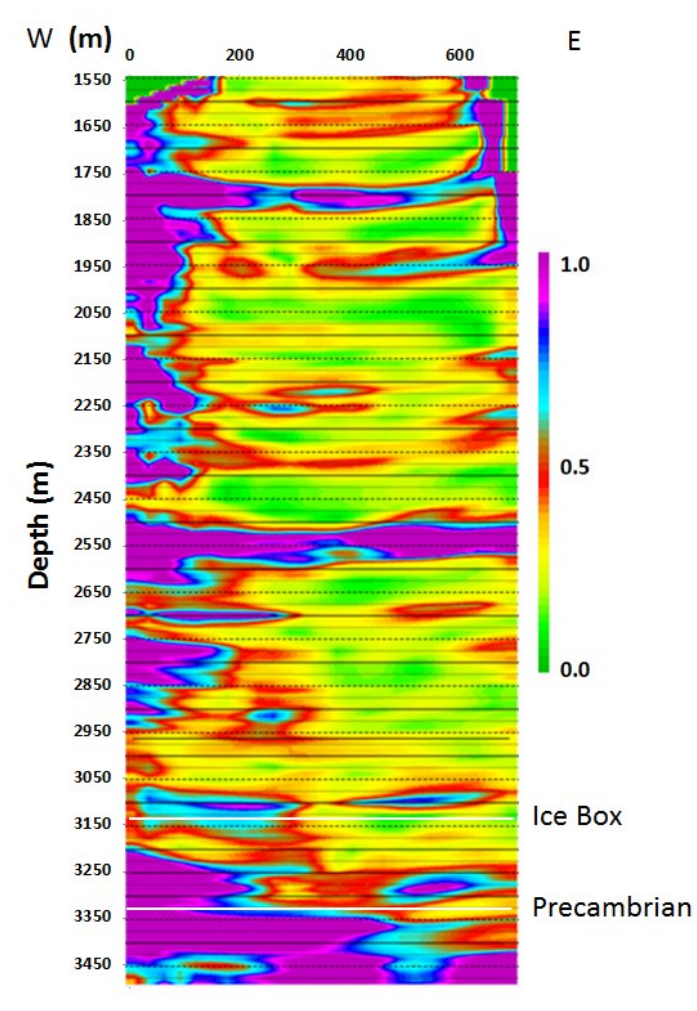

A

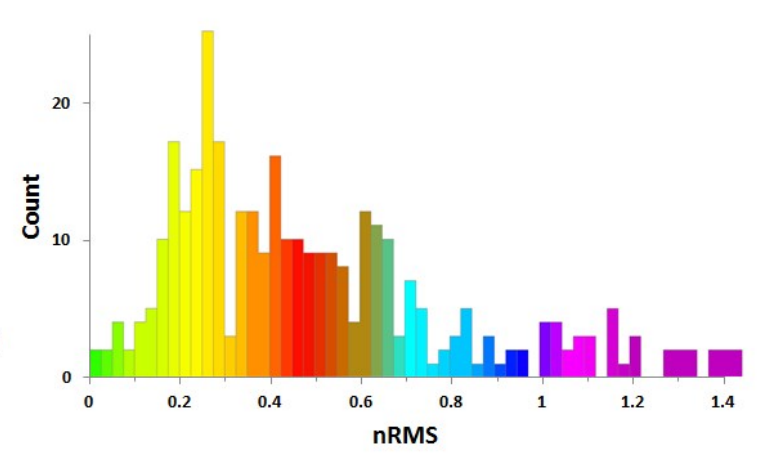

B

Figure 5-7: A) $50 \mathrm{~m}$ window nRMS map of amplitude differences between shots 45364016 and 45603968 of the November 2013 pre-injection baseline survey. Observation well is located at $0 \mathrm{~m}$. B) Histogram of nRMS values shown in A.

\subsubsection{Seismic modeling}

Seismic modeling has been conducted to assess how well the $\mathrm{CO}_{2}$ plume may be imaged at various stages of the injection process. Time-lapse VSP differences were calculated for a range of cumulative $\mathrm{CO}_{2}$ quantities and were compared against the levels of expected repeatability from the field data to assess the likely effectiveness of timelapse VSP monitoring. 
The seismic model used was based on the results of flow simulations through a petrophysical model constructed by the Energy \& Environmental Research Center (EERC). The petrophysical model constructed for the site is a stratigraphic representation (7.6 x $7.6 \mathrm{~m}$ cells with varying depth dimensions) spanning $34 \mathrm{~km}^{2}$ and was built using well log information and 3D surface seismic data. Layers within the reservoir zone comprised 2D stochastic realizations of porosity and permeability (Peck et al. 2014).

Flow simulations performed by the EERC (EERC, 2014) used the Computer Modelling Group (CMG) fluid flow simulation software to determine pressure and fluid saturation distributions within the reservoir. The injection scenario considered is for 3 years of injection at 301t/day as it best corresponds to the proposed injection schedule. Flow simulation results for injection periods of 90 days, 1 year, 2 years, and 3 years are evaluated, corresponding to cumulative injection volumes of $27 \mathrm{kt}, 110 \mathrm{kt}, 220 \mathrm{kt}$ and $330 \mathrm{kt}$ of $\mathrm{CO}_{2}$, respectively. Figure 5-8 shows the $\mathrm{CO}_{2}$ saturation modeled over the 3 years of injection. The injected $\mathrm{CO}_{2}$ is concentrated in three depth intervals $(\mathrm{A}, \mathrm{B}, \mathrm{C}$ in Figure 5-8) within the reservoir ranging in thickness from 20 to $60 \mathrm{~m}$. In each interval, the width of the $\mathrm{CO}_{2}$ plume and the $\mathrm{CO}_{2}$ saturation increase with total quantity of $\mathrm{CO}_{2}$ injected. The uppermost plume (interval A) is relatively narrow (240-560 m) and has generally low-to-moderate $\mathrm{CO}_{2}$ saturations. The $\mathrm{CO}_{2}$ plumes in intervals $\mathrm{B}$ and $\mathrm{C}$ are much wider (680-1680 m) and have moderate-to-high $\mathrm{CO}_{2}$ saturations. The lowermost interval (C) accepts the most $\mathrm{CO}_{2}$.

The flow simulation results were then used to assemble the seismic models in GOCAD software, which involved assigning P and S-wave velocities and bulk densities. The 3D model had dimensions of 3500x3500x3700 m resulting in 326 million $25 \mathrm{~m}^{3}$ 
voxels. The baseline seismic model (i.e., corresponding to the initial reservoir state) was constructed utilizing $\mathrm{P} / \mathrm{S}$-wave velocities and densities from sonic and density log measurements above the reservoir. The sonic log was known to be unreliable from 0-975 $\mathrm{m}$, and therefore P-wave velocities were computed from VSP first arrivals, and S-wave velocities were calculated assuming a $\mathrm{Vs}: \mathrm{Vp}$ ratio of $1: 1.73$ in this range. In the reservoir, velocity and density values were computed from porosity via a recursion relation. Seismic models were constructed for each of the injection periods listed above. The changes in seismic properties due to variable $\mathrm{CO}_{2}$ saturation in the reservoir were determined using the Gassmann model for fluid substitution (Gassmann, 1951; Smith et al., 2003). The properties of impermeable reservoir shale layers were left unchanged in all scenarios.

A 3D finite-difference seismic modeling algorithm, SOFI3D (Bohlen, 2002), was used to generate synthetic data corresponding to the same source-receiver geometry as in the pre-injection baseline VSP survey. The input parameters are listed in Table 5-2. Shot gathers were calculated for each point source along north-south and east-west lines, as recorded by 554 downhole sensors. These sensors were spaced $5 \mathrm{~m}$ from each other and spanned the same depth range as the DAS fibers. The computation time for each shot gather was $\sim 1$ hour running on a CRAY cluster utilizing 490 CPUs. 


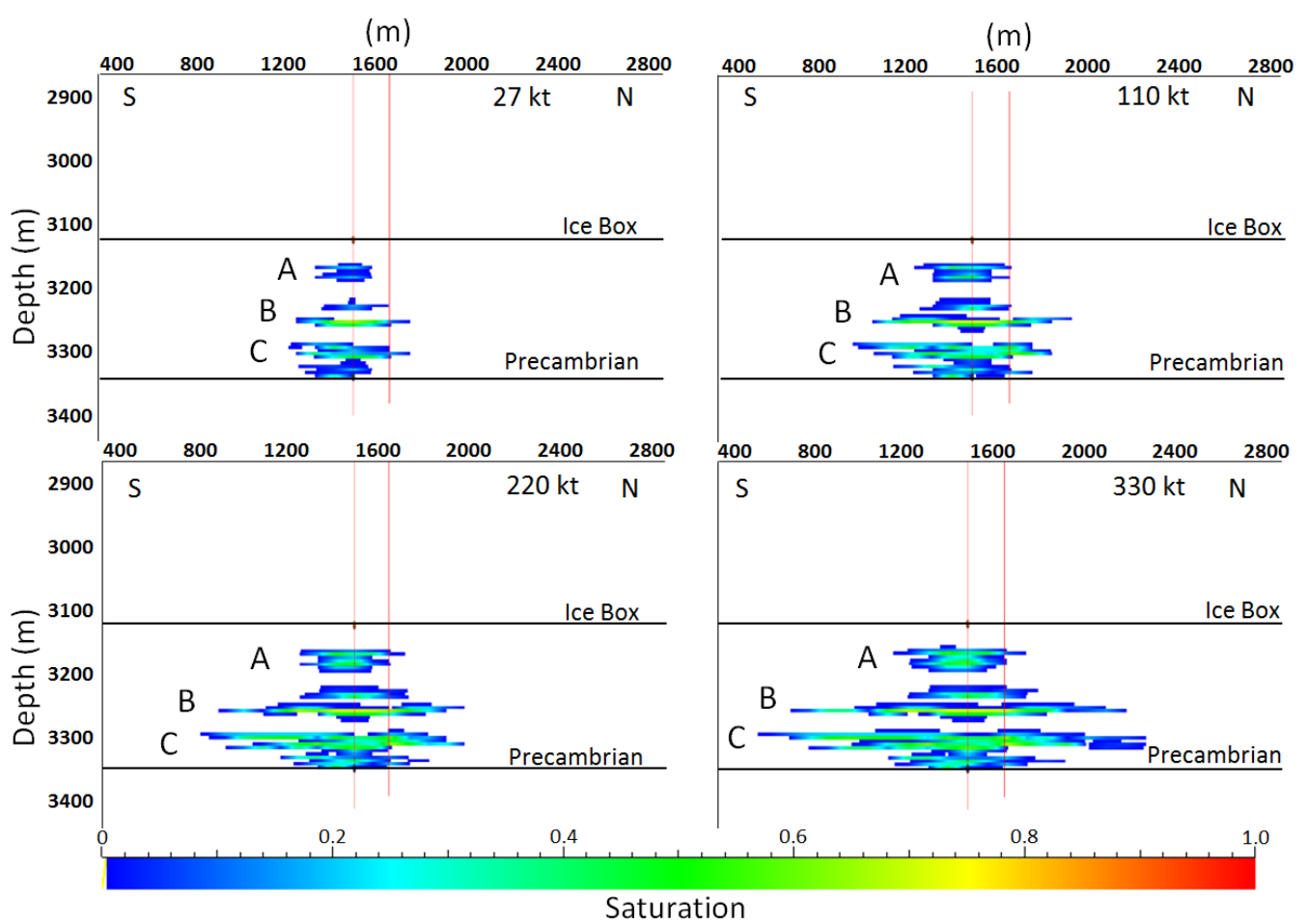

Figure 5-8: Modeled $\mathrm{CO}_{2}$ saturation (fractional) in reservoir after 90 days, 1 year, 2 years, and 3 years of $\mathrm{CO}_{2}$ injection at a rate of $301 \mathrm{t} /$ day (from top-left to bottom-right). Injection well on the left, observation well on the right. 
Table 5-2: Finite Difference Model Parameters (Modified from Melanson, 2015)

\begin{tabular}{|c|c|}
\hline Software & SOFI3D \\
\hline Finite-difference Coefficients & $\begin{array}{l}\text { Holberg, } 8^{\text {th }} \text { order spatial operator, } 2^{\text {nd }} \text { order } \\
\text { temporal operator }\end{array}$ \\
\hline Minimum Velocity & $\mathrm{P}: 2450 \mathrm{~m} / \mathrm{s}, \mathrm{S}: 1416 \mathrm{~m} / \mathrm{s}$ \\
\hline $\begin{array}{l}\text { Dimensions in Voxels (East, North, } \\
\text { Depth) }\end{array}$ & $700,700,740$ \\
\hline Voxel Resolution (East, North, Depth) & $5 \mathrm{~m}, 5 \mathrm{~m}, 5 \mathrm{~m}$ \\
\hline Number of Receivers & 554 \\
\hline Recorded Particle Motions & Particle Velocity $(\mathrm{x}, \mathrm{y}, \mathrm{z})$ \\
\hline Wavefield Propagation Time & $3 \mathrm{~s}$ \\
\hline Timesteps, Sample Period & $10000,0.0003 \mathrm{~s}$ \\
\hline Source Signal & $\begin{array}{l}\text { Ricker wavelet, point source, } 38 \mathrm{~Hz} \text { center } \\
\text { frequency }\end{array}$ \\
\hline Absorbing Boundary & $\begin{array}{l}\text { Perfectly-matched layers, } 30 \text { voxels, } 8 \% \text { decay per } \\
\text { voxel }\end{array}$ \\
\hline Outputs & VSP Shot-gathers, Logs \\
\hline
\end{tabular}



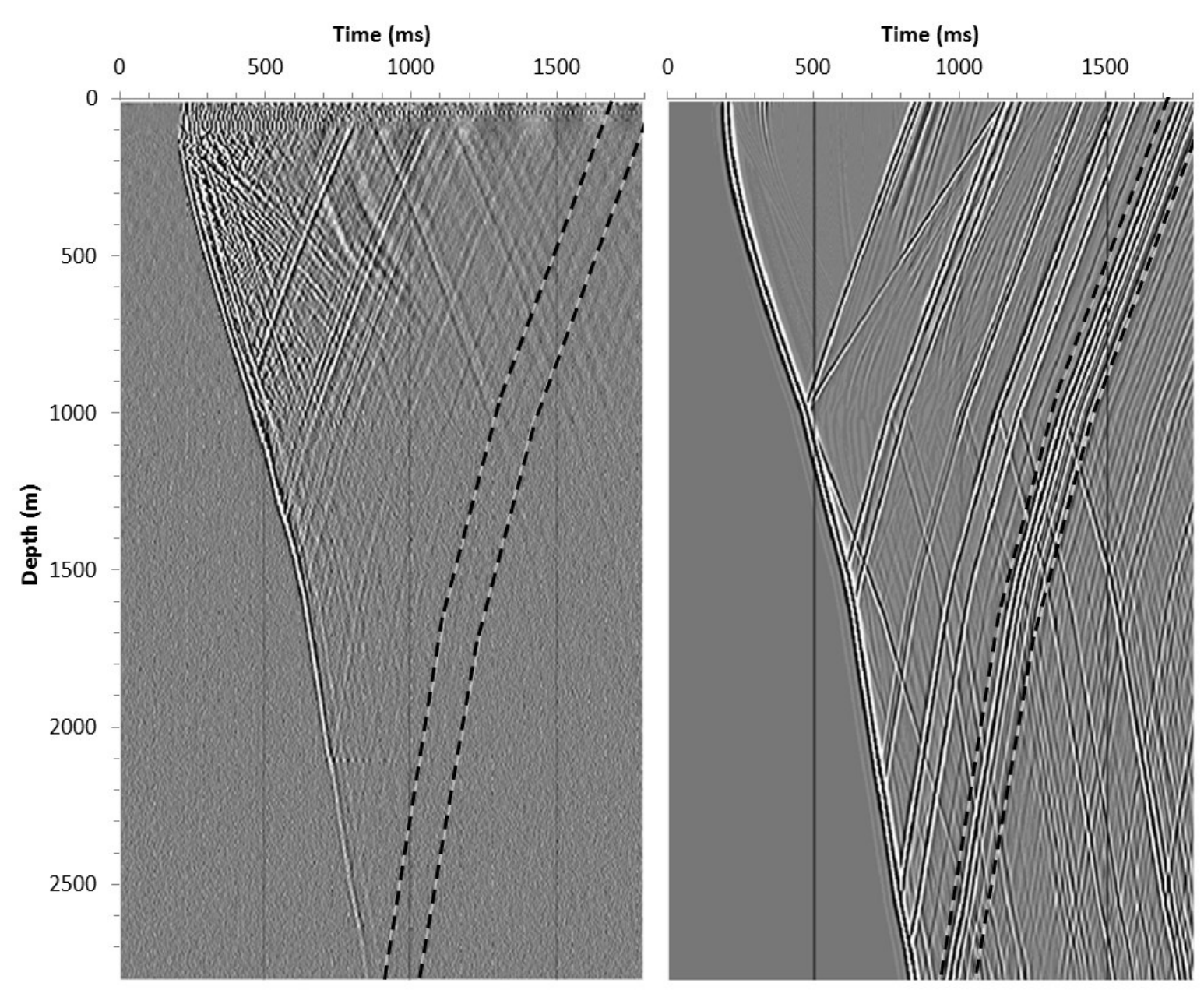

Figure 5-9: DAS $400 \mathrm{~m}$ offset shot gather recorded in the field (left) versus equivalent synthetic shot gather (right). Probable reservoir reflections bound by dotted lines.

The raw synthetic shot gathers (see Figure 5-9 for an example) from these simulations were $f$ - $k$ filtered to remove downgoing energy and S-waves, followed by 2D Kirchhoff depth migration and stacking. Multiples appear below the reservoir reflections (Figure 5-9 (right)), as there is no primary reflectivity within the basement in the models.

A north-south shot line (Figure 5-1) traversing the injection well was selected for comparisons between the different synthetic time-lapse VSP images. The baseline image was subtracted from each of the post-injection images to delineate the signature of the 
$\mathrm{CO}_{2}$ plume over time. Then nRMS maps were computed in the reservoir, using a $10 \mathrm{~m}$ sliding window, to be taken as a measure of the RMS contrast between the baseline and post-injection reservoir.

Figure 5-10A shows the migrated baseline image obtained from 15 synthetic shots spaced at $\sim 144 \mathrm{~m}$ along the north-south line. The reservoir is imaged out to a radius of $\sim 900 \mathrm{~m}$ from the borehole, and down to a depth of $3450 \mathrm{~m}$ (well into the Precambrian basement). The seismic image is calibrated against a well-based synthetic seismogram (black trace in Figure 5-10) to ensure accurate depth registration of the image. At the reservoir level, prominent reflections from the top (Icebox shale at $\sim 3120 \mathrm{~m}$, negative peak) and bottom (Precambrian at $\sim 3350 \mathrm{~m}$, positive peak) of the reservoir correlate well. However, note that the reflection from the bottom of the reservoir apparently extends beneath the Precambrian interface (to $\sim 3400 \mathrm{~m}$ ). This is due to the sidelobes associated with the band-limited seismic wavelet: i.e., it is a result of limited vertical resolution. Furthermore, there is also apparent reflectivity observed at greater depth in the seismic image even though the seismic model contains no changes in impedance below the Precambrian boundary $(3350 \mathrm{~m})$. These are the result of intrabed multiples and potentially incomplete cancellation of converted phases (e.g., P-to-S conversions) during data processing. The image is poorest near the well at depths below the bottom of the recording array where a 'blind spot' occurs which is inherent to the VSP imaging geometry for offset source locations (e.g., Dillon and Thomson, 1984). This effect can be countered by widening the migration aperture, however it would be at the expense of SNR when applied to field data (Yilmaz, 1987). 

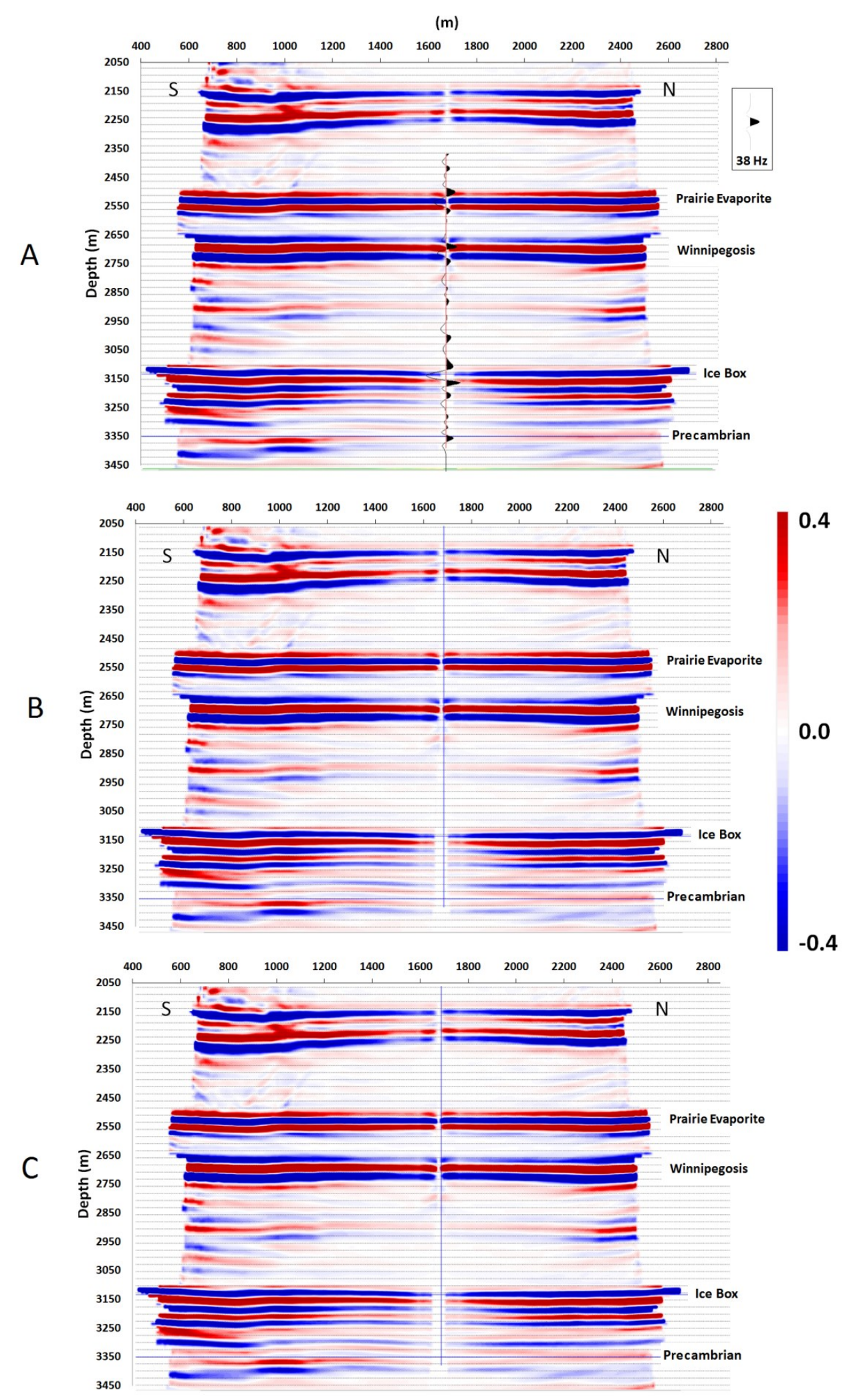

Figure 5-10: (caption next page) 
Figure 5-10: Migrated synthetic images, showing seismic amplitude (scaled particle velocity), of the north-south line shown in Figure 5-1 with reservoir boundaries. A) Baseline image. The observation well is marked by a vertical line overlain with a single seismic trace computed by convolving the sonic log from the observation well with a $38 \mathrm{~Hz}$ Ricker wavelet. B), C) Post-injection images after 2 and 3 years, respectively.

Figure 5-10 $(\mathrm{B}, \mathrm{C})$ compares the baseline VSP image at the reservoir level with comparable images for the cases of 2 and 3 years of $\mathrm{CO}_{2}$ injection. Visual inspection of these images shows that, even in the presence of large quantities of $\mathrm{CO}_{2}$, changes are very subtle. To accentuate the dissimilarities, the corresponding time-lapse difference images for each of the injection times are shown in Figure 5-11. As expected, there are no amplitude differences above the reservoir $(<3120 \mathrm{~m})$ and most of the amplitude residual is confined to the reservoir zone (3120-3350 m). Marked amplitude differences in the depth interval immediately below the Precambrian interface (3350-3400 m) are artifacts associated with the sidelobes of the Precambrian reflection as explained above. Smaller amplitude differences at greater depth are most likely due to changes in the intra-reservoir multiples (travel times and amplitudes) that appear within the basement due to their longer travel times and the incomplete removal of converted-wave energy during migration and stacking. Throughout the injection period, the amplitude residuals tend to increase most prominently in the bottom and below the reservoir and, after 3 years, the plume reaches the lateral extent of the illuminated zone.

Figure 5-12 shows the nRMS maps of the amplitude residuals. After $27 \mathrm{kt}$ of $\mathrm{CO}_{2}$ injection, nRMS values in the reservoir near the borehole exceed 1.0. Then RMS values steadily increase until $330 \mathrm{kt}$ of $\mathrm{CO}_{2}$ injection where $\mathrm{nRMS}>1.0$ as far as $700 \mathrm{~m}$ south of 
the borehole. The largest nRMS anomalies in Figure 5-12 occur below the zone $(>3400$ m) where we expect to see the effects of $\mathrm{CO}_{2}$ presence in the reservoir (see label D). As can be seen on plots of RMS amplitude differences (Figure 5-13), this zone corresponds to an interval of very small amplitude differences. These small amplitude differences are accentuated in the plot of nRMS amplitude differences as they are normalized by correspondingly small amplitude reflections (Figure 5-10) in this zone. This demonstrates the importance of assessing nRMS values in combination with RMS values. 


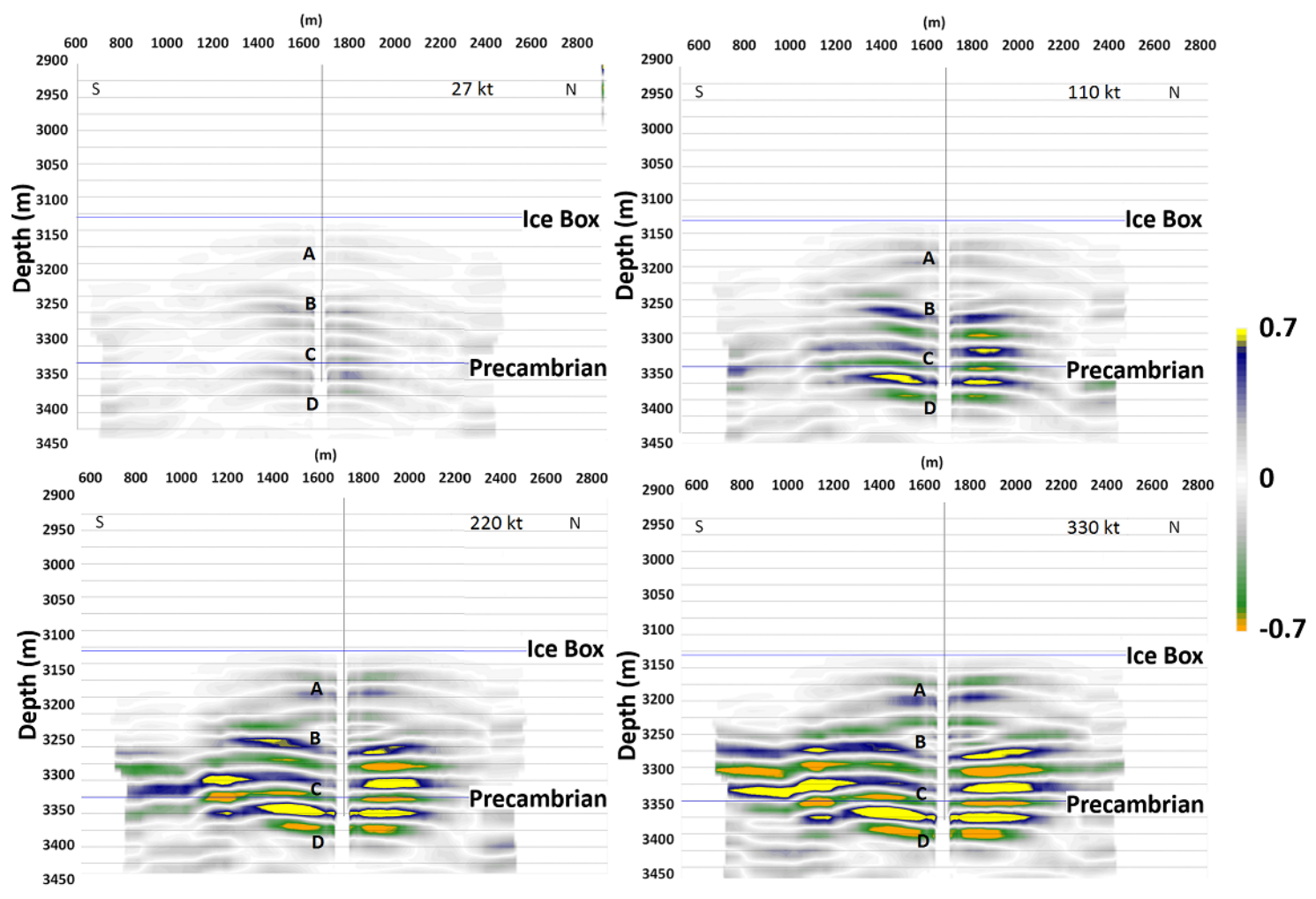

Figure 5-11: Migrated synthetic time-lapse difference images after 90 days, 1 year, 2 years, and 3 years of $\mathrm{CO}_{2}$ injection at a rate of $301 \mathrm{t} /$ day (from top-left to bottom-right). Amplitude differences are scaled by a factor of $\sim 6$ relative to Figure $5-10$. Stratification of the plume is indicated by four labels, A-D (Compare with Figure 5-8). 
(m)

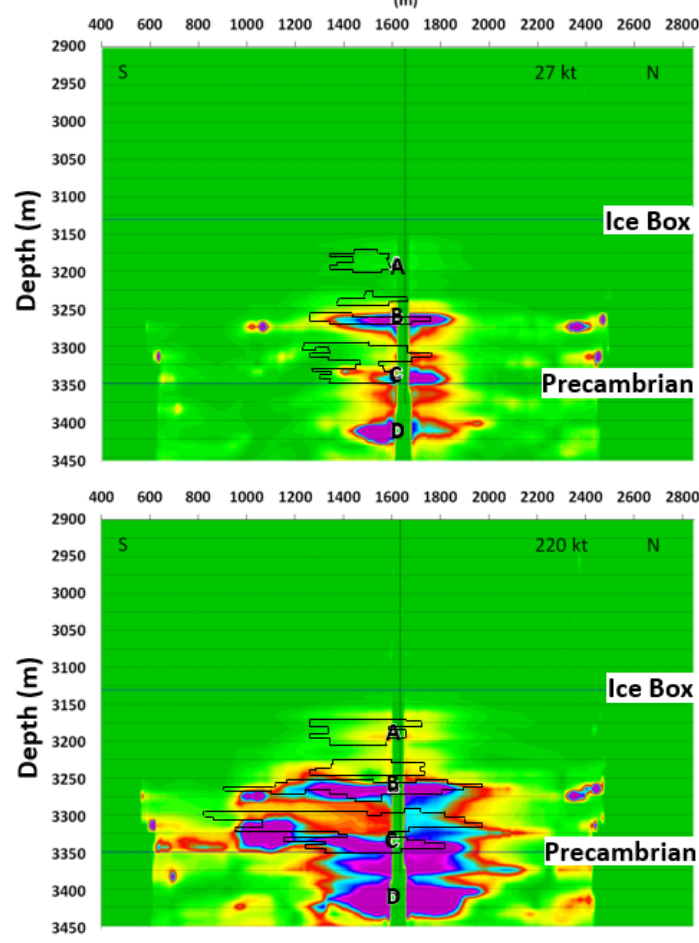

(m)

$\begin{array}{lllllllllllll}400 & 600 & 800 & 1000 & 1200 & 1400 & 1600 & 1800 & 2000 & 2200 & 2400 & 2600 & 2800\end{array}$

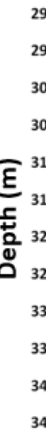

2900
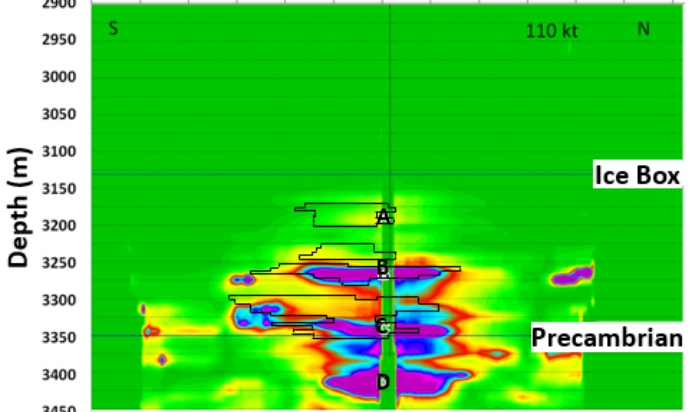

3450

$\begin{array}{lllllllllllll}400 & 600 & 800 & 1000 & 1200 & 1400 & 1600 & 1800 & 2000 & 2200 & 2400 & 2600 & 2800\end{array}$

1.0

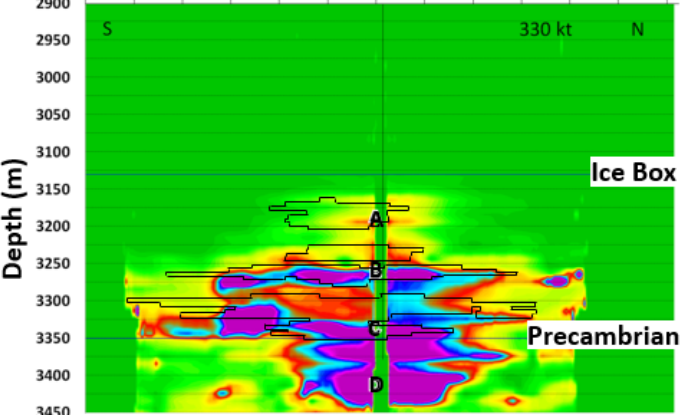

Figure 5-12: $10 \mathrm{~m}$ window nRMS maps of the amplitude residuals of the time-lapse

difference images shown in Figure 5-11. Plume locations from Figure 5-8 are shown as black outlines. 

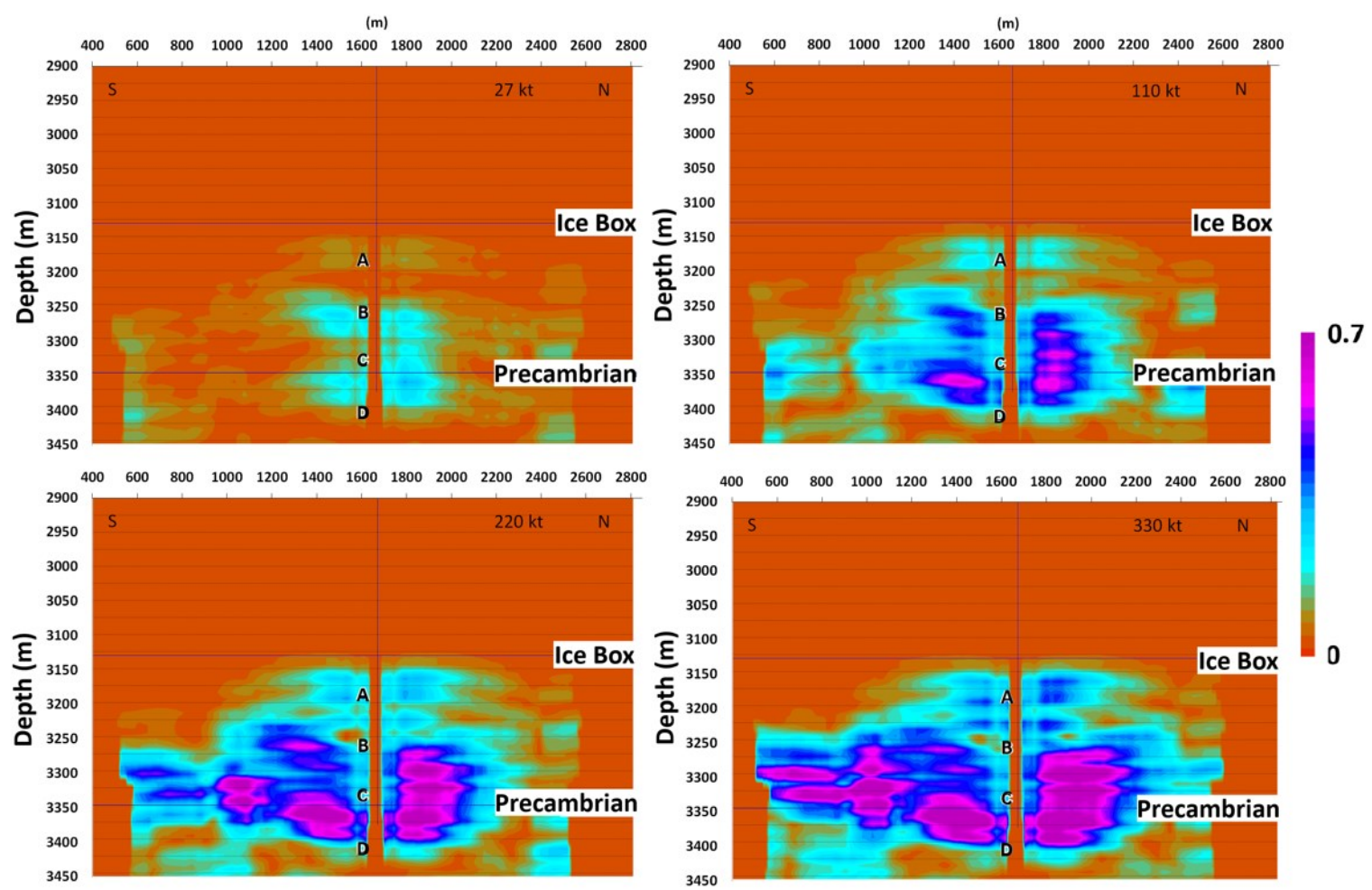

Figure 5-13: $10 \mathrm{~m}$ window RMS maps of the amplitude residuals of the time-lapse difference images shown in Figure 5-11. Note that amplitudes are scaled by a factor of $\sim 6$ relative to

Figure 5-10.

\subsection{Discussion}

The nRMS maps of amplitude differences (Figure 5-12) can be compared with the modeled $\mathrm{CO}_{2}$ distributions (Figure 5-8). To facilitate the comparison, outlines of the $\mathrm{CO}_{2}$ plumes from Figure 5-8 are superposed in Figure 5-12. The stratification of $\mathrm{CO}_{2}$ within distinct high-permeability zones (depths A, B, C) and the width of these zones are well captured in the seismic images, albeit with poorly defined borders. The uppermost zone A is the least prominent in the seismic images due to its low porosity and resulting relatively lower $\mathrm{CO}_{2}$ saturations. Low associated nRMS values imply that only under excellent repeatability conditions will this zone be imaged. Zones B and C have associated large 
nRMS values indicating that they will be well imaged even with moderate repeatability. The apparent continuation of the zone C nRMS anomaly below the Precambrian surface is primarily due to a limitation in seismic resolution; i.e., the $38 \mathrm{~Hz}$ zero-phase wavelet used in modelling has a dominant wavelength of $\sim 100 \mathrm{~m}$ at the reservoir depth (compare with Figure 5-10A). The significance of Zone D in the nRMS image is discounted based on a comparison with the corresponding RMS image which shows this is a zone characterized by very low amplitudes.

Repeatability estimates from the real field data (Figure 5-7) suggest that injectioninduced nRMS differences will have to exceed $\sim 0.3$ in order to be visible. The time-lapse difference nRMS values (Figure 5-12) in the reservoir associated with the presence of $\mathrm{CO}_{2}$ generally exceed this value for each of the four injection volumes considered. After 90 days $\left(27 \mathrm{kt} \mathrm{CO}_{2}\right)$, zones $\mathrm{B}$ and $\mathrm{C}$ meet this visibility criteria near the borehole, whereas zone A will likely not be visible until 1 to 2 years $\left(110 \mathrm{kt}\right.$ to $\left.220 \mathrm{kt} \mathrm{CO}_{2}\right)$. It appears that saturation values above $\sim 0.3$ are well imaged in Figure 5-11 and Figure 5-12, and consistently meet the visibility criteria.

It should be noted that the repeatability inferred from the RMS values calculated in this study is likely underestimated for several reasons: 1) The comparison presented is for single shot gathers, so random noise likely contributes far more than for a multi-fold (i.e., stacked) image that is the end product of standard data processing; 2) outliers in the difference images can be exaggerated in nRMS images if the amplitudes are regionally low; and 3) the shots were located $1000 \mathrm{~m}$ from the observation well, and thus have lower SNR than closer shots which would be included in producing a multi-fold VSP image. 
Due to the fact that the shots were not collocated, but $\sim 10 \mathrm{~m}$ apart, there is an expected deviation in signal that was not quantified in this study.

The repeatability of dynamite shots in the vicinity of the Aquistore site has been demonstrated in previous studies. White et al. (2015) report a mean nRMS value of 0.55 and standard deviation of 0.17 for raw data from 261 repeat shot gathers that were recorded by a permanent surface array of 630 geophones at the Aquistore site. The baseline and monitor surveys were acquired in March 2012 and May 2013, respectively. Roach et al. (2015) achieved a global nRMS value of 0.07 for migrated stacks of these data. The nearby Weyburn field which employed dynamite sources also maintained good repeatability (nRMS $0.30-0.34$ ) for at least 4 surveys conducted over a period of 7 years (White, 2013). We expect that comparably low nRMS values may be achieved for fully processed, multi-fold time-lapse VSP images for the Aquistore site. If this is the case, then the plume would meet the visibility criteria for its entire volume for any of the cases shown above.

The advantages of traditional $3 \mathrm{C}$ geophones as compared to DAS for VSP recording include a higher frequency response, higher SNR, and omni-directional sensitivity. However, DAS has the advantage of providing an order-of-magnitude increase in the number of sensors and a larger recording aperture which results in imaging over a larger depth interval. The latter is certainly an advantage for $\mathrm{CO}_{2}$ monitoring where surveillance of the layers overlying the reservoir is essential. DAS also has the advantage of requiring less effort in field deployment as compared to wireline geophones particularly in the case where fiber cables were permanently installed during well-completion. In this study, DAS SNR (and hence data repeatability) prove to be 
adequate for the purposes of time-lapse imaging in spite of the lower sensitivity of DAS as compared to traditional geophones. As next generation DAS technology is developed and post-processing methods improve, the sensitivity discrepancy between DAS and traditional geophones will hopefully be reduced.

\subsection{Conclusions and Outlook}

Synthetic time-lapse VSP amplitude differences have been calculated for fluidflow simulations of $\mathrm{CO}_{2}$ injection in a deep reservoir for quantities of $\mathrm{CO}_{2}$ ranging from $27 \mathrm{kt}$ to $330 \mathrm{kt}$. Comparison of the associated nRMS values against DAS data repeatability estimated from actual field data indicates that individual decameter-thick $\mathrm{CO}_{2}$ layers in the reservoir should be visible for total injection quantities of as little as 27 kt of $\mathrm{CO}_{2}$. Imaging of the $\mathrm{CO}_{2}$ layers should be robust for the lower part of the reservoir as this is where the majority of the injected $\mathrm{CO}_{2}$ resides, resulting in strong seismic differences. In contrast, $\mathrm{CO}_{2}$ within the upper reservoir occurs at lower saturations and in

a lower porosity zone with the result that visibility of the plume will likely be confined to a volume near the borehole until later in the injection timeline. Due to the ability of VSP to image near the wellbore with greater frequency range and SNR as compared to surface seismic, VSP will be employed to monitor the $\mathrm{CO}_{2}$ during the early stages of injection, whereas surface surveys will likely be used in later phases to characterize the greater expanse of the $\mathrm{CO}_{2}$ plume. At future stages of processing and imaging, comparisons will continue to be made with the surface seismic results to evaluate the assumption that VSP will provide better plume characterization in the reservoir. The first post-injection VSP survey is tentatively scheduled for March 2016, when the reservoir is expected to contain more than $27 \mathrm{kt}$ (90 days) of $\mathrm{CO}_{2}$. In future seismic analysis, it is clear that measures 
should be taken to better image the $\mathrm{CO}_{2}$ plume. This includes characterizing and removing inter-bed multiples, wavelet-processing to attenuate outer lobes, time-variant time shifts to account for the altered velocity profile introduced by the $\mathrm{CO}_{2}$, and close attention to the removal of undesirable wave-modes. Also, it will be necessary to expand the processing and imaging to $3 \mathrm{D}$.

\subsection{Acknowledgements}

The authors would like to thank the Petroleum Technology Research Centre and and Kyle Worth in particular. for project management, and SaskPower. We would also like to acknowledge J. A. Hole for the use of the 3D eikonal solver. Brian Roberts, Lisa Roach and Michelle Robertson are thanked for their fieldwork at Aquistore. The seismic data were acquired by Silixa, Schlumberger Carbon Services, Tesla Exploration, Geospace Technologies and the Geological Survey of Canada. We would also like to thank Chevron for their contributions. This is publication 20150496 of the Geological Survey of Canada. 


\section{Imaging the Aquistore Reservoir after 36 kilotonnes of $\mathrm{CO}_{2}$ Injection using Distributed Acoustic Sensing}

\subsection{Abstract}

Aquistore is a geological $\mathrm{CO}_{2}$ storage project that is utilizing a deep saline formation as a storage reservoir. From April 2015 to February 2016, approximately 36 ktonnes of $\mathrm{CO}_{2}$ were injected into the reservoir at a depth of $3130-3350 \mathrm{~m}$. This study presents the analysis of distributed acoustic sensing (DAS) 3D VSP data acquired in February 2016, marking the first seismic survey since injection began. The VSP data were processed in parallel with baseline pre-injection data from a November 2013 survey, with the objective of detecting and characterizing the subsurface $\mathrm{CO}_{2}$ plume, and evaluating the repeatability of DAS in a reservoir monitoring project. A single processing sequence was devised that (1) accurately imaged the reservoir for both the baseline and monitor data, and (2) attained adequate repeatability to observe time-lapse differences related to the presence of $\mathrm{CO}_{2}$. Repeatability was somewhat compromised by the less advanced noise cancellation methodology of the DAS system used for the baseline survey. In the final cross-equalized migrated data volumes, normalized root-mean-square (nRMS) difference values of $<0.4$ were attained at the reservoir level indicating good repeatability compared to most surface seismic studies. An injection-related amplitude anomaly with maximum nRMS values of $\sim 0.7$ is apparent in the Deadwood Formation of the reservoir, whereas no significant nRMS anomalies were observed near the injection and monitoring wells in the Black Island or above the reservoir. 


\subsection{Introduction}

Aquistore is a geological $\mathrm{CO}_{2}$ storage project whose study site is located near the town of Estevan in southeastern Saskatchewan, Canada (Figure 6-1). $\mathrm{CO}_{2}$ is supplied from the nearby SaskPower Boundary Dam Power Station where it is captured and transported via pipeline to the Aquistore injection well. The $\mathrm{CO}_{2}$ is injected into a sandstone formation at $\sim 3200 \mathrm{~m}$ depth for the purpose of long-term storage. The Aquistore project is employing 4D vertical seismic profiling (VSP) to characterize the shape, size, and location of the injected $\mathrm{CO}_{2}$ plume over time. A central aspect of the project pertains to the choice of downhole receivers utilized for the 4D VSPs. Optical fiber-based seismic technology has rapidly improved over the past 15-20 years (Bostick, 2011) to the point where sensors now have the sensitivity and resolution required for deep reservoir monitoring (Mateeva et al. 2014). Having obtained encouraging results for comparative studies of VSP data acquired using both downhole geophones and a distributed acoustic sensing (DAS) system connected to an optical cable cemented in an observation well (Harris et al., 2016), it was decided that ongoing 4D VSP monitoring at the Aquistore site would be conducted only using the DAS system. Here, we present the first DAS-based 4D VSP results obtained by processing the first monitor VSP survey (from February 2016) and a pre-injection baseline VSP survey (from November 2013) in parallel. At the time of the first monitor survey 36 ktonnes of $\mathrm{CO}_{2}$ had been injected into the reservoir, an amount which Harris and co-workers (2016) had predicted should be observable in the monitor VSP data. A particular challenge in matching the characteristics of the two data vintages was due to technological improvements to the 
DAS system in the 2 years between surveys. The 4D VSP results will be compared to those of a 4D surface seismic survey also acquired in February 2016 (Roach et al. 2017).

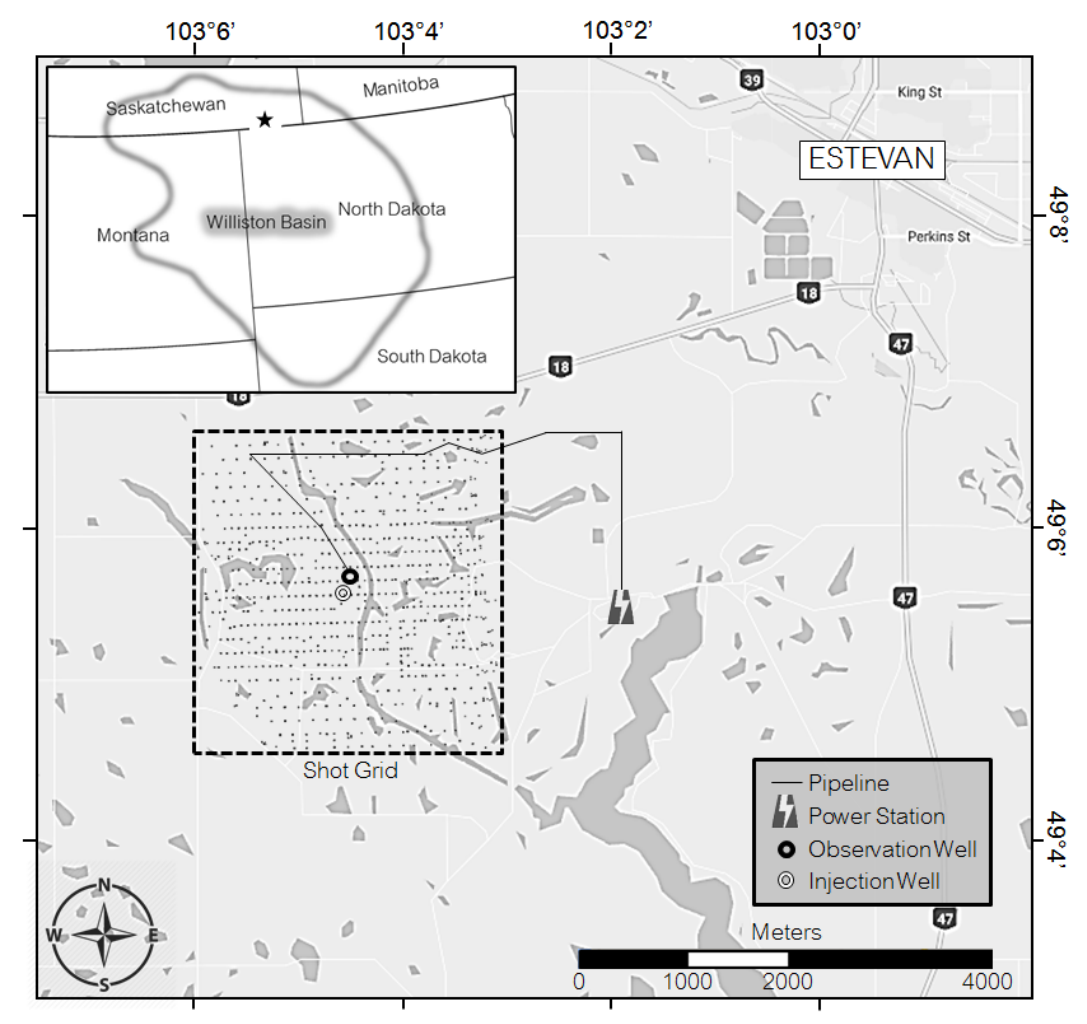

Figure 6-1: Location map of the Aquistore $\mathrm{CO}_{2}$ storage site and survey area (in box delineated by dashed line).

\subsubsection{Background}

Several advantages associated with VSP often make it a preferable method to surface seismic during early stages of $\mathrm{CO}_{2}$ injection. These advantages are primarily a result of shorter reflection travel paths that are thus subjected to less attenuation, potentially resulting in an overall higher signal to noise ratio $(\mathrm{S} / \mathrm{N})$ and broader frequency content (O'Brien et al., 2004). Since the area that can be imaged using VSPs is smaller than what is possible to achieve with surface receiver arrays (Hardage, 2000), surface 
seismic methods become more suitable when the lateral expanse of a $\mathrm{CO}_{2}$ plumes exceeds what is practical for VSP.

Harris and co-workers (2016) established the feasibility of using DAS-based 3D VSP to map the $\mathrm{CO}_{2}$ plume in the Aquistore reservoir for quantities as small as 27 ktonnes. Modeling showed that a comparison with pre-injection results would yield normalized root-mean-square (nRMS) amplitude differences in excess of 1.0 that extend farther than $200 \mathrm{~m}$ from the injection well. An estimate of time-lapse noise was obtained from field recordings by analyzing differences in the VSP data for very closely located shots after processing and imaging. The study showed that even on a single-shot basis, the nRMS noise floor was generally lower than 0.7 at the reservo ir level. For comparison, White et al. (2015) determined an average nRMS of 0.57 for raw surface-based shots recorded by a permanent array of surface geophones, and Roach et al. (2015) attained a global nRMS of 0.07 for stacked and migrated volumes from these same data. A global nRMS value of 0.2 would therefore be expected if a similar improvement was attained with the VSP data. Although DAS is expected to be associated with high repeatability due to the permanent nature of its installation, this has not been previously demonstrated.

Direct comparisons have been made of VSP data recorded simultaneously at the Aquistore site using both downhole geophones and DAS (Harris et al., 2015; Harris et al., 2016; Miller et al., 2016). These comparisons have demonstrated that, although traditional three-component geophones recorded data with a higher $\mathrm{S} / \mathrm{N}$ and a larger bandwidth, the DAS system provided an order of magnitude increase in spatial sampling (i.e., trace spacing) and a far larger recording aperture. An aperture that permits the imaging of both the reservoir and overlying structures obviates the need for repositioning 
the wireline throughout the survey. During the imaging process, it became apparent that the lower S/N of the DAS data was compensated for by the $\sqrt{n}$ (where $n$ is the number of samples) reduction of noise in the resultant stack image afforded by the denser spatial sampling.

\subsubsection{The Aquistore $\mathrm{CO}_{2}$ storage site}

The Aquistore site is located within the Williston Basin (Figure 6-1), which spans southern Saskatchewan, southwestern Manitoba, eastern Montana, most of North Dakota, and northwestern South Dakota. Containing an abundance of coal and salt seams, depleted oil and gas reservoirs, and saline formations, the basin is a suitable candidate for large-scale carbon storage. It is situated in a tectonically stable environment, and underlies a plethora of existing stationary $\mathrm{CO}_{2}$ sources (Wright et al., 2013). The basin has hosted previous $\mathrm{CO}_{2}$ injection projects including Weyburn-Midale (e.g., Duxbury et al., 2012; White, 2013), Northwest McGregor (Sorensen et al., 2011), and the Lignite Field Validation Test site in Burke County (Botnen et al., 2009). After the Alberta Basin, the Williston Basin is the second most significant basin for $\mathrm{CO}_{2}$ storage in Canada.

The Aquistore $\mathrm{CO}_{2}$ storage reservoir comprises the Winnipeg and Deadwood formations that unconformably overlie the Precambrian basement. The reservoir is a 200 m thick sequence of porous, brine-filled sandstones and shales, capped at a depth of $\sim 3130 \mathrm{~m}$ by the $\sim 15 \mathrm{~m}$ thick Icebox shale unit. Evaporites of the Prairie Formation form $\mathrm{a} \sim 150 \mathrm{~m}$ thick secondary seal at a depth of $\sim 2500 \mathrm{~m}$. Generally, the reservoir formations are characterized by high permeabilities of up to $1000 \mathrm{mD}$ and moderate porosities of 11-17\% (Whittaker and Worth, 2011); however these formations exhibit somewhat lower porosity and permeability at the Aquistore location (Figure 6-2). The injection well 
is perforated at 4 depths in the reservoir to allow infiltration into 3 sandstone formations. There is one perforation zone in the Black Island Member (Winnipeg Formation, 3175 m), two in the upper Deadwood Formation (3230 and $3255 \mathrm{~m}$ ), and one in the lower Deadwood Formation (3300 m).

A suite of well-logs from the injection well is shown in Figure 6-2, and have been tied to a corridor stack from a $150 \mathrm{~m}$ offset shot. The gamma-ray curve shows alternating sandstone and shale units throughout the reservoir, suggesting stratification and segregation of the $\mathrm{CO}_{2}$ distribution. Upper units in the reservoir exhibit sharper impedance contrasts than do most of the Deadwood interfaces. Consequently, stronger reflections are produced in the corridor stack for the Black Island. Several closely spaced units in the Deadwood are not well captured in the corridor stack due to the lower impedance contrasts and limited vertical resolution of the survey. It is therefore expected that the ability to localize the plume will be somewhat reduced for these units.

Injection commenced in April 2015, with sustained injection rates of 400-600 t/day since fall of 2015 (Worth et al., 2015). At the time of the first monitor survey in February 2016, approximately 36 ktonnes of $\mathrm{CO}_{2}$ had been injected into the reservoir. 

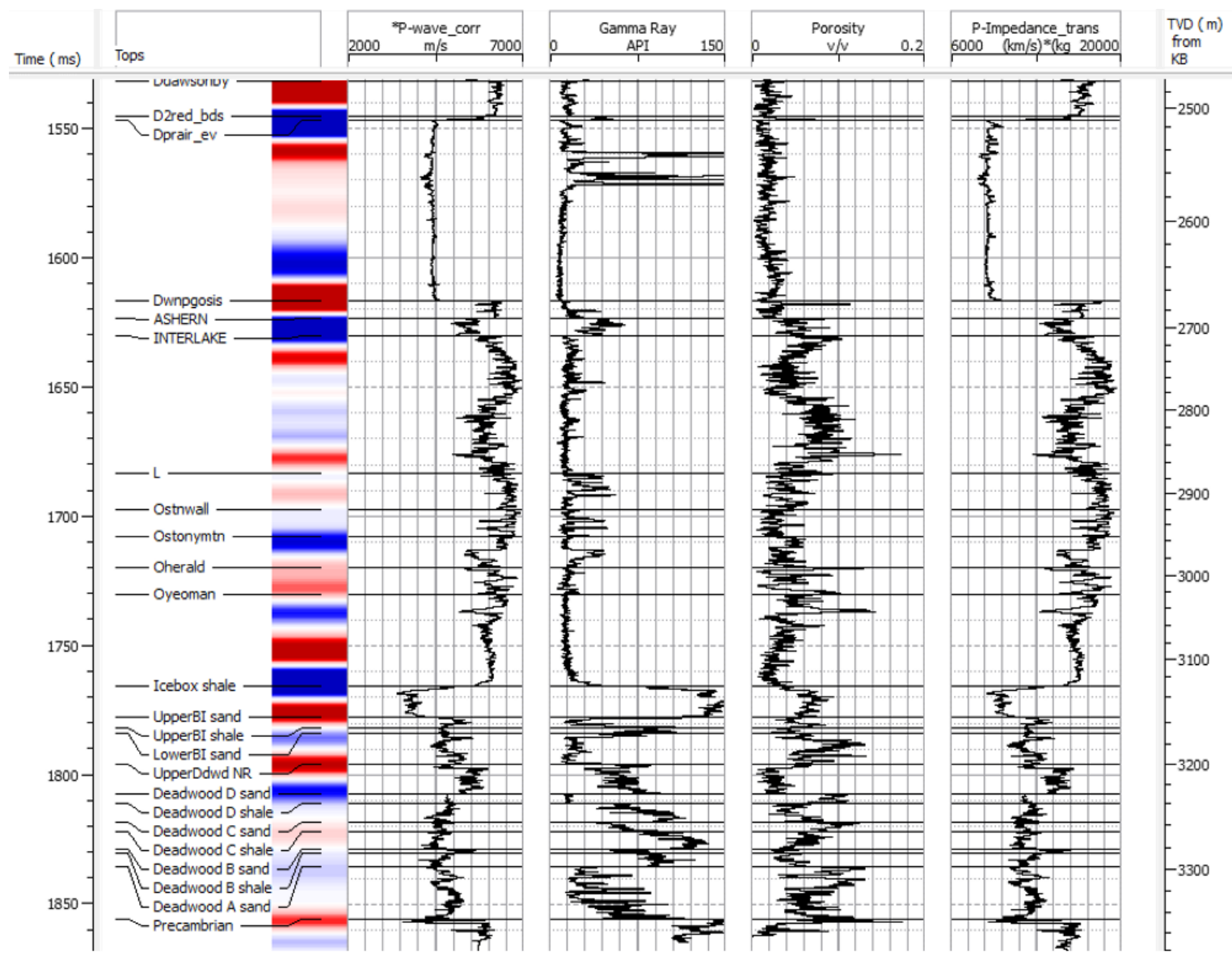

Figure 6-2: Corridor stack of near-offset DAS shot gather (left) and injection well logs. Time is plotted linearly and depths (below kelly bushing) in the logs are stretched to align with reflections.

\subsubsection{Distributed Acoustic Sensing}

DAS is a technology that relates Rayleigh backscattering of light pulses in a fiber optic cable to the localized strain rate applied to the cable via acoustic energy. This allows the fiber itself to be employed as a near-continuous array of sensors (Mestayer et al., 2011; Miller et al., 2012). Nearly any commercially available telecommunications fiber can be utilized for this purpose in combination with a DAS interrogator unit, provided that the fiber is coupled (via friction or pressure) to the surrounding medium. 
Existing downhole fibers used for distributed temperature sensing (DTS) can be used as well. The interrogator unit emits and detects pulses of coherent light that travel the twoway length of the fiber. Localized strain on the fiber induced by a seismic wavefield causes a perturbation in the travel path of the backscattered light, and can be converted to depth on the basis of the time-of-flight of the reflected pulses. Repeated, rapid pulses of light allow the measurement of fiber deformation over time, and can be converted to the induced strain rate (Mestayer et al., 2011; Hornman, 2016). The choice of pulse length determines the "gauge length" of the sensor system. A measurement corresponds to the average optical path perturbation along a gauge length. It is not necessary for the sensor spacing to be equal to the gauge length, i.e., adjacent sensors may overlap in the sense that they interrogate overlapping lengths of fiber (Parker et al., 2014). However, it is necessary for the gauge length to be much smaller than the minimum seismic wavelength. Generally, a longer gauge length is associated with higher $\mathrm{S} / \mathrm{N}$ but at the expense of spatial resolution and distorted high frequencies (Mateeva et al., 2014). In a "dual-pulse" system, two pulses are emitted in succession and the gauge length corresponds to the distance between the pulses. A path length perturbation is obtained by measuring the deviation between the two backscattered pulses (Hornman, 2016).

One of the primary advantages of the DAS system is the ability to achieve very dense spatial sampling as compared to traditional geophone arrays although the DAS signal for each sample point represents a local average over the 'gauge length' in contrast to geophones which provide point measurements (Shatalin et al., 1998; Mestayer et al., 2011; Miller et al., 2012; Daley et al., 2016). Spatial sample intervals of 5 m, 2 m, and 1 $\mathrm{m}$ are common with DAS. DAS only requires (though is not limited to) a single fiber 
(Bostick, 2000; Hornby and Burch, 2008). Cables can be installed in slim holes and can reside permanently downhole, eliminating the need for repeat deployments, thereby reducing survey costs. A permanent deployment contributes to the repeatability of 4D surveying making DAS a suitable choice for reservoir monitoring, especially when considering the full vertical coverage that is afforded by a fiber spanning the entire length of the well casing (Mateeva et al., 2014). Permanent deployment also reduces the risk of tool or wellbore damage as repeat surveys are completely non-intrusive. However, for installations exterior to the casing, there is an inherent risk of compromising well integrity and potentially creating leakage paths in a CCS project.

At present, the sensitivity and S/N of DAS has not yet matched that of traditional electro-mechanical sensors such as geophones. However, as will be made apparent in this study, the performance of DAS over the last two years has improved greatly. Further improvement is possible in the future as more of the fibers' available optical bandwidth will be utilized for interrogation (Mateeva et al., 2014). Currently, DAS is most effectively employed in VSP P-wave analysis. This is a result of a limitation of the fiber itself, as sensitivity is restricted to its longitudinal axis. Efforts to mitigate this limitation are ongoing, and focus on winding the fiber in a way that presents an orthogonal surface to incident wavefields at any angle, i.e., a helix or a twisted sinusoid (Lumens et al., 2013; Mateeva et al., 2014; Freifeld et al., 2016; Ning and Sava, 2016). 


\subsection{Methods}

\subsubsection{Data acquisition}

All VSP data at the Aquistore site have been acquired in an observation well that is located $\sim 150$ m north-northeast of the $\mathrm{CO}_{2}$ injection well. The observation well was drilled and completed in November 2012. Two multimode (for DTS) and two single mode fibers were encased in $6.35 \mathrm{~mm}$ diameter stainless steel tubing clamped to the outside of the well casing and cemented in place during well completion. The fiber cable was damaged during installation resulting in a truncated sensor array functional to a depth of $2766 \mathrm{~m}$ of the total $3400 \mathrm{~m}$.

In May 2013, preliminary DAS recordings were taken in conjunction with a baseline surface seismic survey. Encouraging results prompted a full baseline 3D DAS survey in November 2013. Three VSP data sets were obtained simultaneously during the baseline survey using multimode and single mode DAS, and a traditional geophone wireline. Comparisons between these data can be found in Harris et al. (2016) and Miller et al. (2016).

For both the baseline and monitor surveys, the DAS data were acquired with a Silixa iDAS interrogator unit using a $10 \mathrm{~m}$ gauge length, and $2.036 \mathrm{~m}$ sensor spacing (Table 6-1). Over the $2766 \mathrm{~m}$ depth interval, this yielded 1383 traces per shot. Depths were determined along the fiber by performing a tap test (tapping on the wellhead to calibrate optical depths) prior to the surveys, and were cross-referenced to strong reflections observed in the two vintages of data. Preprocessing of the raw optical data to produce seismic data was performed by Silixa LTD. In both surveys, each $2.036 \mathrm{~m}$ measurement was the average of 8 consecutive $0.25 \mathrm{~m}$ spaced readings. During the 
baseline survey, a simple unweighted average was used for noise cancellation whereas an optimally weighted average was used in the monitor survey resulting in comparably greater reduction of noise (Miller et al., 2016). The native response of the iDAS system is proportional to strain rate, and may be converted to absolute strain rate in a linear fashion (Miller et al., 2016). Strain rate units are expressed as nm/m/s.

Table 6-1: Acquisition parameters for baseline (November 2013) and monitor (February 2016) DAS surveys

\begin{tabular}{ll}
\hline Parameter & Shots \\
\hline \hline Sources & 1 kg dynamite \\
Grid size & 670 shots over $3 \times 3 \mathrm{~km}$ \\
Shot spacing & $72 \times 144 \mathrm{~m}$ (inline x crossline) \\
Shot depth & $15 \mathrm{~m}$ \\
& Sensors \\
Interrogator & iDAS \\
Fiber & Single mode VHS 3000 \\
Array length & $2766 \mathrm{~m}$ \\
Spacing & $2.036 \mathrm{~m}$ \\
Gauge length & $10 \mathrm{~m}$ \\
Sampling frequency & $1000 \mathrm{~Hz}$ \\
Trace length & $4 \mathrm{~s}$ \\
Native response & Strain rate \\
\hline \hline
\end{tabular}

The planned nominal shot grid consisted of 924 shot locations and a 60 -level geophone array deployed in the interval between $1650 \mathrm{~m}$ and $2650 \mathrm{~m}$ depths, attaining a minimum fold of 30 over the targeted area assuming $36 \times 36 \mathrm{~m}$ bin size. In practice, the grid was reduced to 670 shot locations spanning $3 \times 3 \mathrm{~km}$, at inline and crossline spacings of $72 \mathrm{~m}$ and $144 \mathrm{~m}$, respectively (Figure 6-3). Inline spacings were increased to $144 \mathrm{~m}$ for larger offsets. In part, this grid reduction was a cost-saving measure. Furthermore, many of the nominal shot locations were inaccessible due to uneven terrain or water bodies, 
which resulted in either elimination or relocation of these shots. Both surface and VSP surveys employed $1 \mathrm{~kg}$ dynamite charges buried at a depth of $15 \mathrm{~m}$. Shot station locations were resurveyed post-drilling for the monitor survey; they were not resurveyed for the baseline survey.

As shot holes were redrilled for each survey, there was a degree of compromise in the choice of source locations necessitated by infrastructure and environmental conditions (Figure 6-3). In the 27 months between the baseline and monitor surveys, new fences and a $\mathrm{CO}_{2}$ pipeline were installed, which required shifting certain shot locations by up to a maximum of $4.5 \mathrm{~m}$. More significantly, the vicinity near the injection and observation wells became host to work trailers, new infrastructure, and working personnel during the monitor survey. As a result, near-offset shot positions were shifted as far as $200 \mathrm{~m}$, rendering them unusable for $4 \mathrm{D}$ analysis. The shortest offset shot appropriate for $4 \mathrm{D}$ analysis was $150 \mathrm{~m}$ from the observation well. 


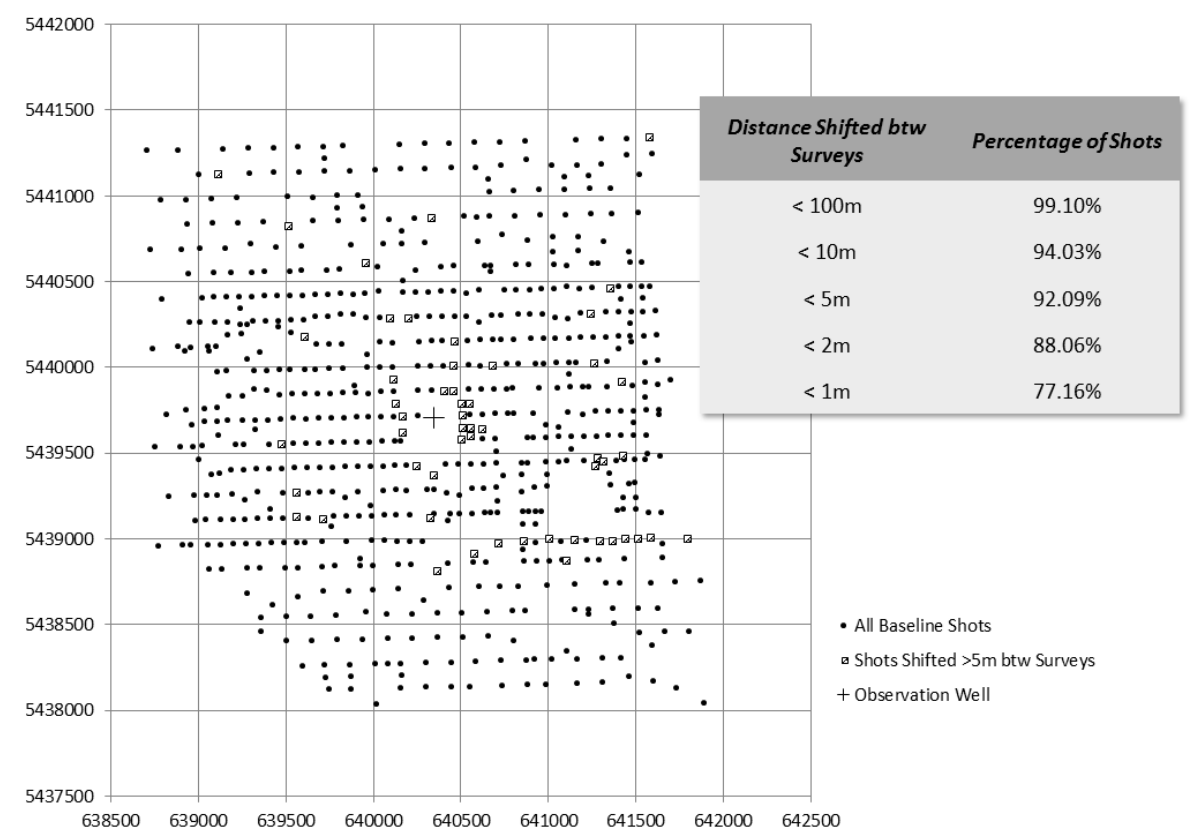

Figure 6-3: VSP baseline shot locations. Squares indicate shot locations that shifted more than $5 \mathrm{~m}$ for the subsequent monitor survey.

\subsubsection{Data processing}

In time-lapse seismic processing, the objective is to maximize the repeatability of data unaffected by $\mathrm{CO}_{2}$ injection. Ideally, differences between subsequent vintages should be dominated by injection- or production-related effects. In reality, difference images are contaminated with non-repeatable noise, and an effective 4D survey must minimize this noise so that injection- or production-related signatures can be observed. In this study, a parallel processing approach (Lumley et al., 2003; Li et al., 2011) is taken, wherein data from separate vintages are processed in identical flows.

A processing flow was devised with the objective of accurately imaging the subsurface while maximizing the overall repeatability between the monitor and baseline images. To assess the degree to which the repeatability criterion is satisfied, the 
normalised root-mean-square (nRMS) of the time-lapse amplitude differences is computed as a metric (Kragh and Christie, 2002; Lumley et al., 2003; Lumley et al., 2008; Li et al., 2011):

$$
n R M S=\frac{2 \times R M S_{M-B}}{R M S_{B}+R M S_{M}}
$$

where subscripts $B$ and $M$ represent baseline and monitor data, respectively. The nRMS has a range of 0 to 2 , with 0 corresponding to perfectly repeatable $4 \mathrm{D}$ data sets, and 2 corresponding to polarity reversed data sets. With modern acquisition and processing techniques, nRMS values for surface seismic data of 0.6 indicate good repeatability and below 0.2 indicate excellent repeatability (Lumley, 2009). Comparable repeatability can generally be expected for VSP data. Frequently, global nRMS values are used as a quality control metric throughout the processing flow, and steps are rejected if they do not decrease this value (e.g., Li et al., 2011). This approach was considered unsuitable in this study for two reasons. Firstly, the $\mathrm{S} / \mathrm{N}$ characteristics between the baseline and monitor vintages were too dissimilar (Figure 6-4). In many of the processing steps, minimizing the nRMS simply amounted to matching the noise floors while amplifying the differences in the signal. Secondly, careful choice of nRMS windows around high $\mathrm{S} / \mathrm{N}$ regions is difficult with prestack VSP data. With surface data, the first problem can be overcome by selecting high $\mathrm{S} / \mathrm{N}$ horizons for nRMS computation. With VSP data, these horizons are difficult to characterize among many shot gathers until the time-domain data are mapped to the depth-domain. Performing this transformation between each processing step is impractical as it requires considerable raytracing and rebinning. Using windows around strong reflections in prestack data was also inappropriate, as the $\mathrm{S} / \mathrm{N}$ was not high enough 
in the baseline data at larger offsets. To evaluate the repeatability in the prestack data throughout the processing sequence, nRMS values were computed by selecting windows that exhibit very high $\mathrm{S} / \mathrm{N}$ around the direct waves in the upper part of the sensor array. This allowed for an evaluation of repeatability on a shot-by-shot basis. Figure 6-5 illustrates two such examples. First break picks were used to define RMS windows around the direct waves to give an estimate of repeatability that is not determined by the change in the noise floor, but by the character of the signal. Reservoir nRMS values were used in the poststack cross-equalization flow as an acceptance criterion, however, on the condition that windows did not overlap with possible plume locations.

To the extent possible, both vintages of data were processed with identical processing sequences and parameters to avoid introducing unrepeatable effects as summarized in Table 6-2. 

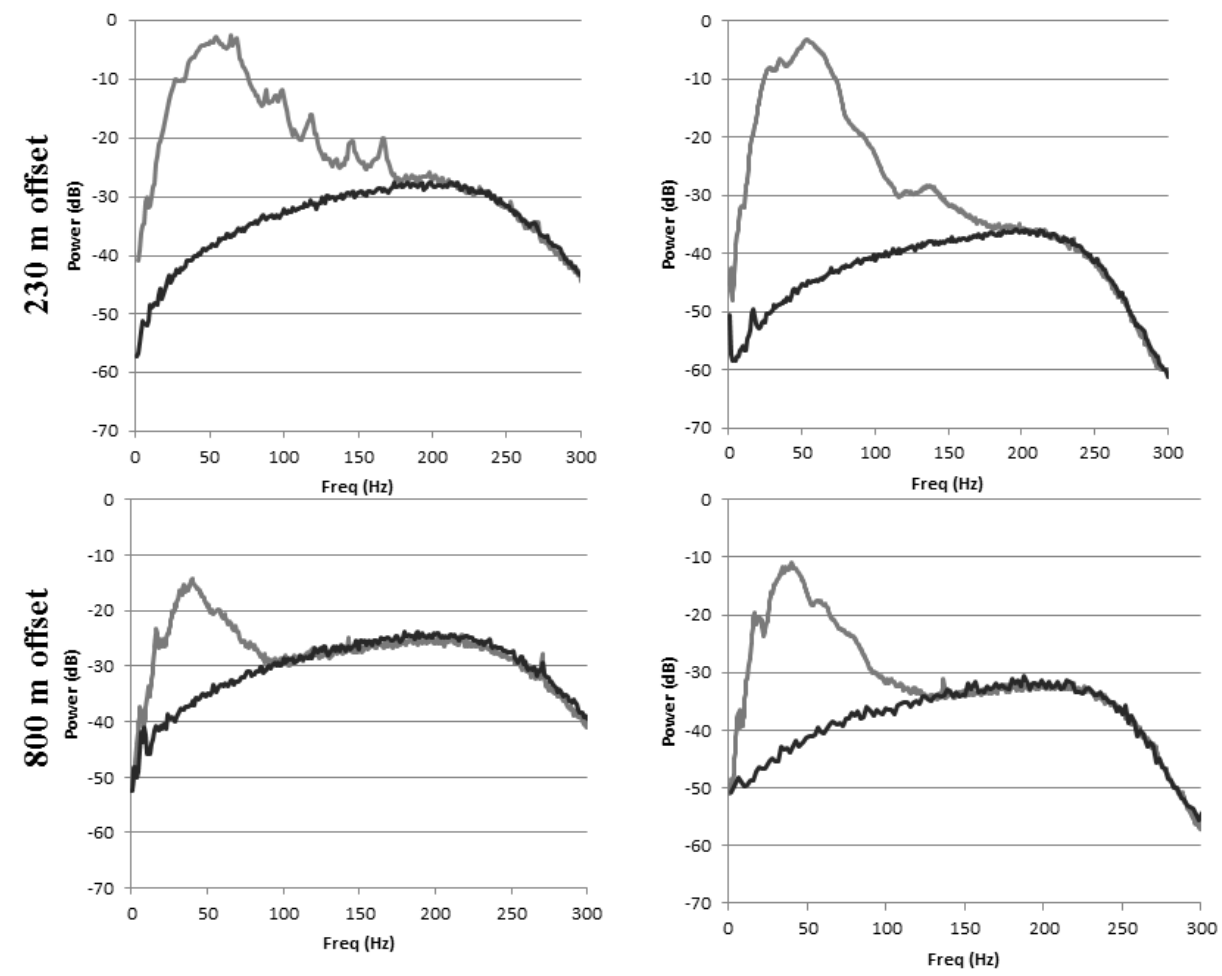

Baseline

Monitor

Figure 6-4: Examples of spectra for two shot gathers from the baseline and monitor surveys.

In the $40-50 \mathrm{~Hz}$ range, the monitor data exhibit $\mathrm{S} / \mathrm{N}$ improvements of up to $10 \mathrm{~dB}$. Windows for signal estimation were taken around upgoing energy in the top $1000 \mathrm{~m}$ of the wireline, and windows for noise estimation preceded the first arrivals for noise estimation. Signal is shown in grey, and noise in black. 

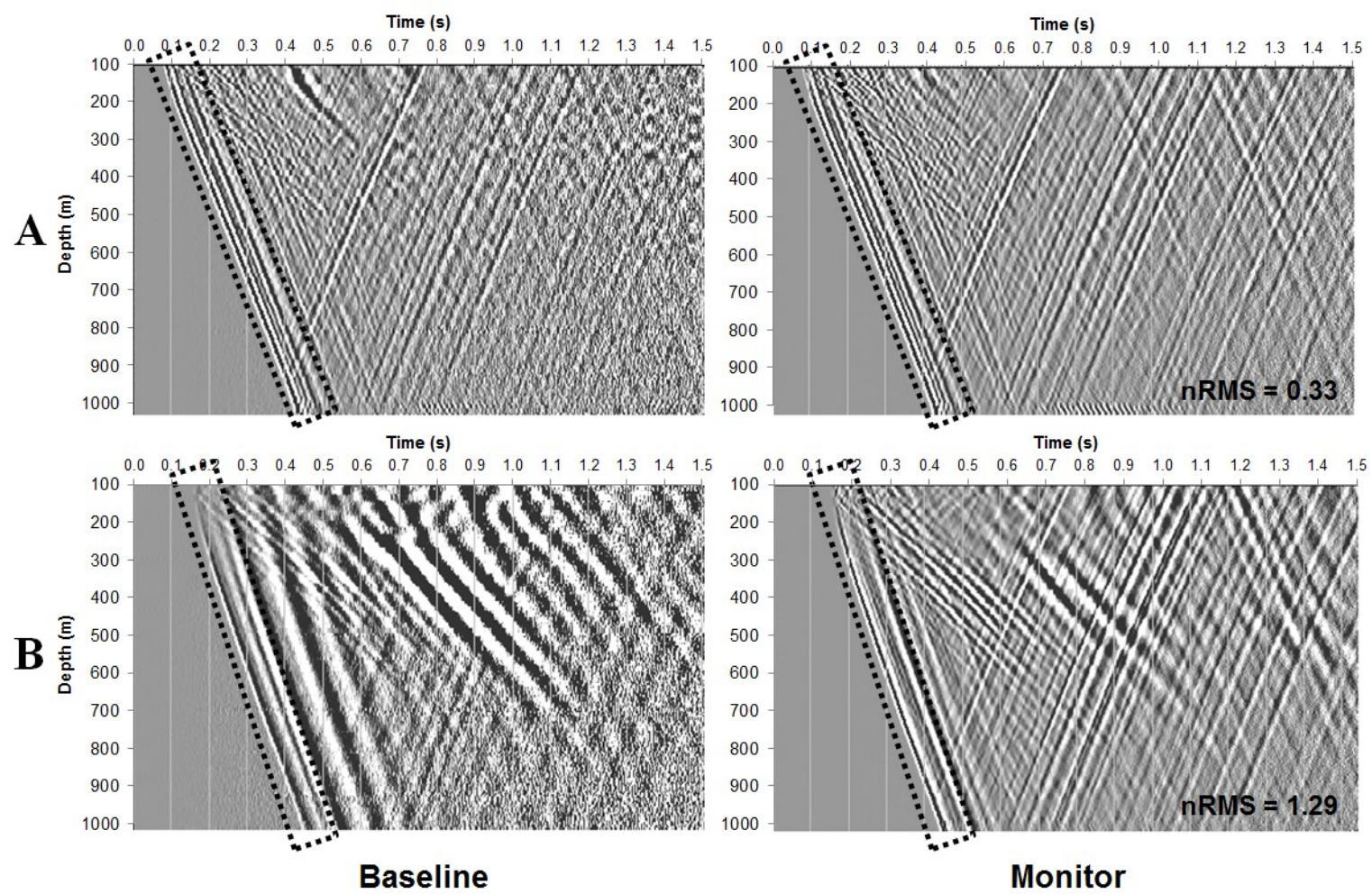

Figure 6-5: Examples of shot gathers from the baseline and monitor surveys exhibiting good (A) and poor (B) repeatability. Values of nRMS were computed by selecting $70 \mathrm{~ms}$ windows around direct waves (box delineated by a dotted lines). Baseline and monitor data are scaled by the same shot-based factor for display. 
Table 6-2: Processing steps and parameters. (*) indicates a process that is data dependent.

\begin{tabular}{|c|c|}
\hline Processing Step & Parameters \\
\hline \multicolumn{2}{|l|}{$\begin{array}{l}\text { Prestack } \\
\text { Trimming and alignment of data sets }\end{array}$} \\
\hline \multicolumn{2}{|l|}{ Polarity reversal of monitor data } \\
\hline $\begin{array}{l}\text { Omission of unrepeatable/shifted shots } \\
\mathrm{t}^{2} \text { amplitude scaling }\end{array}$ & Shifts $>4.5 \mathrm{~m}$ omitted \\
\hline Shot-based amplitude scaling* & Based on RMS of first arrivals (100-1000 m) \\
\hline Median filtering of direct wave* & 51 trace filter \\
\hline \multicolumn{2}{|l|}{$\mathrm{f}-\mathrm{k}$ filtering of converted waves } \\
\hline Shot-based deterministic deconvolution* & $200 \mathrm{~ms}$ filter, $1 \%$ prewhitening, $10-15-80-90 \mathrm{~Hz}$ \\
\hline Zero-phase bandpass filter & 5-10-55-65 Hz Ormsby \\
\hline 3D Kirchhoff depth migration & $\begin{array}{l}12.5 \times 12.5 \times 2 \mathrm{~m} \text { bins, } \pm 15^{\circ} \text { aperture, } \\
\text { identical velocity model for both data sets }\end{array}$ \\
\hline \multicolumn{2}{|l|}{$\begin{array}{l}\text { Poststack } \\
\text { Rho filter and } 50^{\circ} \text { phase shift }\end{array}$} \\
\hline Phase-time matching* & 1500-3100 m design window \\
\hline Shaping filtering* & 1500-3100 m design window \\
\hline Amplitude normalization* & 1500-3100 m design window \\
\hline
\end{tabular}

It was necessary to first trim the monitor data set, as it included a length of fiber along the surface. The channels of the two data sets were depth aligned by ensuring coregistration of the first subsurface traces. The polarity of the monitor data was reversed to match that of the baseline. Any shot that was present in one vintage but not in the other was removed, regardless of the quality of the data therein. Many of the shot locations had been relocated to some extent, particularly for the near-offset shots. The effect of these repositioned shots was investigated to establish a cut-off threshold for position shifts that maintained sufficient repeatability. Figure 6-6 shows data examples for a range of intersurvey shot position shifts. As the overburden is highly variable at the Aquistore site, it was found that there was no well-defined threshold that would guarantee good repeatability. Generally, any shift greater than $4.5 \mathrm{~m}$ was omitted in this study, but many 
of the smaller shifts proved to yield poorly repeatable data as well. This is in part due to a $\sim 3$ m uncertainty of shot coordinates in the baseline survey. Due to this unpredictability, shot gathers were accepted on a shot-by-shot basis rather than including all shots shifted less than $4.5 \mathrm{~m}$.

Another effect that necessitated the omission of data was irreconcilable wavelets between the baseline and monitor surveys (Figure 6-5 (B)). The cause of variations in wavelet shape was likely related to differences in shot coupling. For some shot gathers, this effect could be rectified during deconvolution, but often the baseline shot gathers were too noisy for this procedure to be successful.

Both Figure 6-5 and Figure 6-6 display data that have undergone $t^{2}$ amplitude scaling and shot-based amplitude cross-equalization. No channel-variable scaling was applied in order to best preserve lateral amplitude variations. The most effective technique for cross-equalizing amplitudes between the baseline and monitor data sets was to scale each monitor shot gather by a constant that equates the RMS values in the windows around the direct waves to those of the corresponding baseline gather, i.e., the same windows used for computing nRMS values for repeatability evaluations. The window ends at $1000 \mathrm{~m}$ because including data with low $\mathrm{S} / \mathrm{N}$ below this depth would only serve to match noise floors between surveys (Figure 6-7). The abrupt signal decay at this depth is attributed to reduced coupling due to lower quality of the cement bound. Although the $\mathrm{S} / \mathrm{N}$ is considerably lower $(\sim 3.5)$ below this boundary, reflections from the reservoir are still visible in the shot gathers (as will be shown in Figure 6-14). 

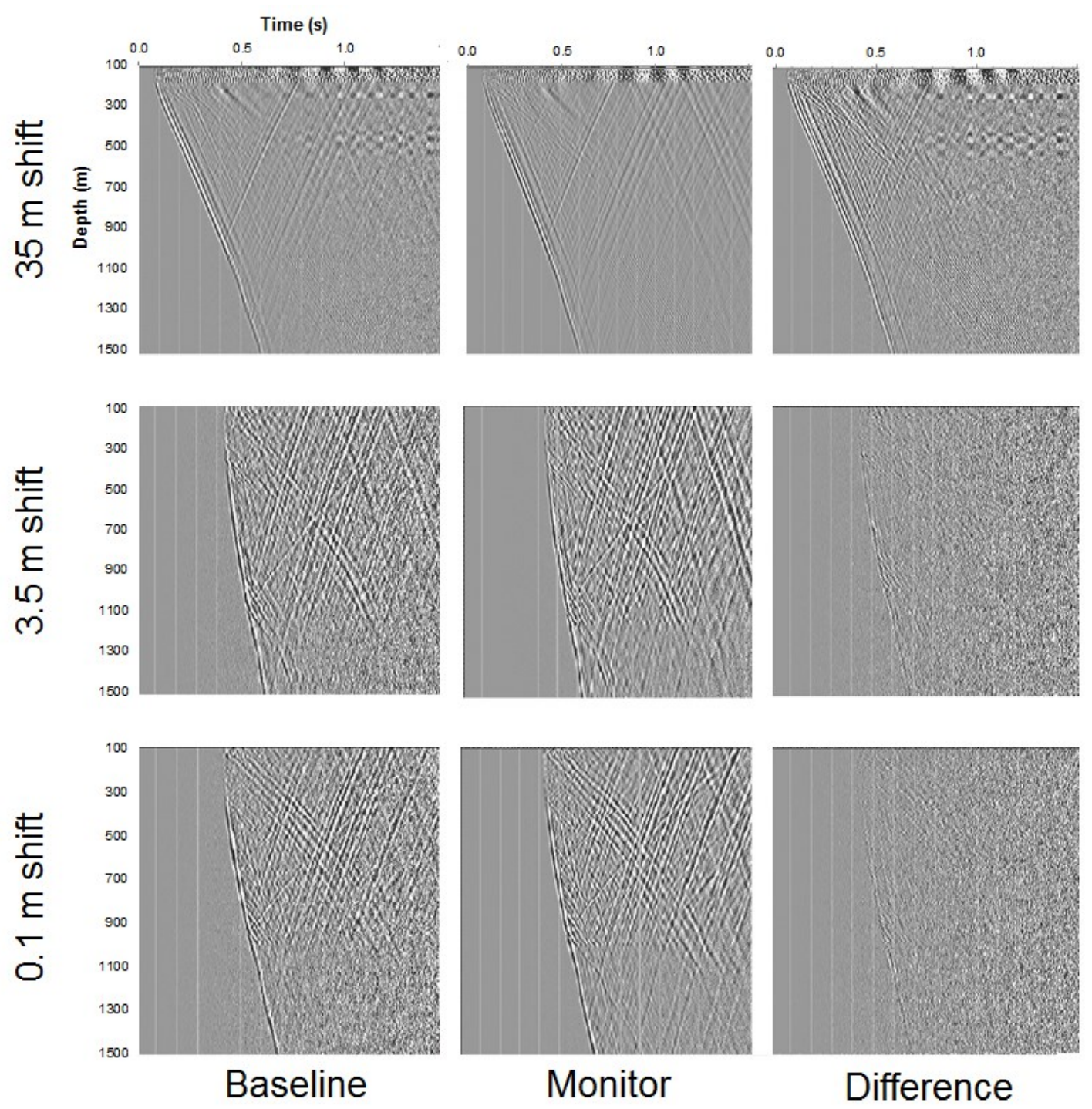

Figure 6-6: Examples of amplitude-equalized shot gathers for shot locations that are different between the baseline and monitor surveys. The $35 \mathrm{~m}$ shifted shot (top) yielded a difference image that is not suitable for further 4D analysis. Generally, shots shifted less than $4.5 \mathrm{~m}$ (middle and bottom) yielded difference images that consisted mainly of noise, and direct Pwaves. The downgoing P-wave energy was filtered out regardless. Baseline, monitor, and difference data are scaled by same shot-based factor for display. 


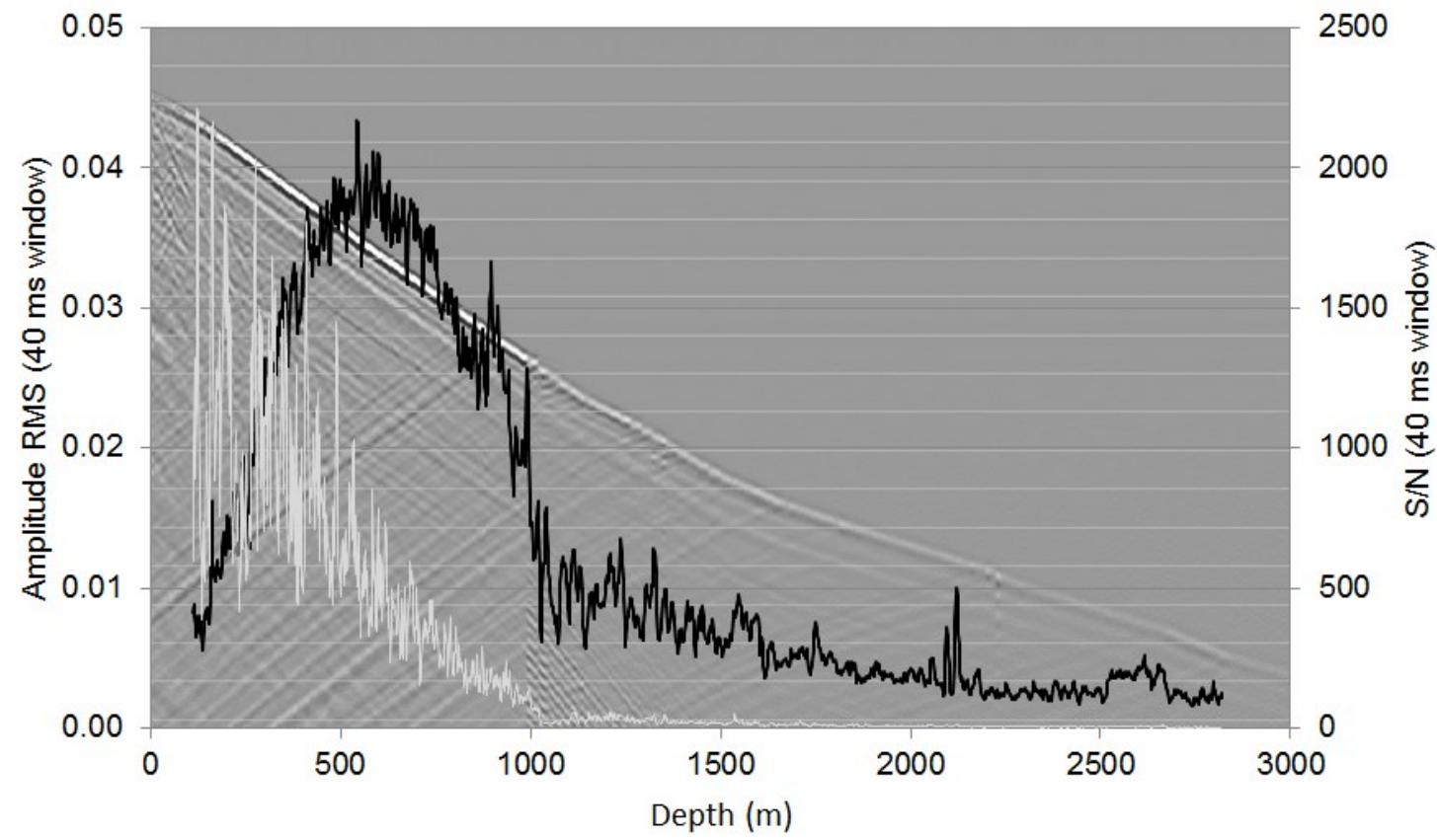

Figure 6-7: Abrupt amplitude and S/N decay of first arrivals at a depth of approximately 1000 $\mathrm{m}$ for a near-offset shot gather. Amplitude RMS values shown in black, and S/N shown in grey. The shot gather shown in the background is from the monitor survey. In the bottom 500 $\mathrm{m}$ of the DAS line, the $\mathrm{S} / \mathrm{N}$ is $\sim 3.5$.

Auto-picking algorithms could reliably pick the first arrivals in the top $1000 \mathrm{~m}$ of the wireline but struggled for the bottom $1800 \mathrm{~m}$ due to the decay in signal strength. This issue was addressed by employing a raytracing algorithm to compute first arrivals for the nearest-offset shot gather, which were used to seed the auto-picking algorithm. Shot gathers were then sorted by offset, and each shot used the first arrivals from the previous shot to seed its auto-picking process.

The direct waves were most effectively removed via a 51 -trace median filter/subtraction. Additional velocity filtering was performed with a series of $f$ - $k$ filters. Filters were not designed separately for each shot gather, but were designed for certain 
ranges of offsets, i.e., shot gathers were distributed into groups according to offset and shared a common $f-k$ filter. Each filter was designed with the objective of removing the converted energy visible in Figure 6-8.

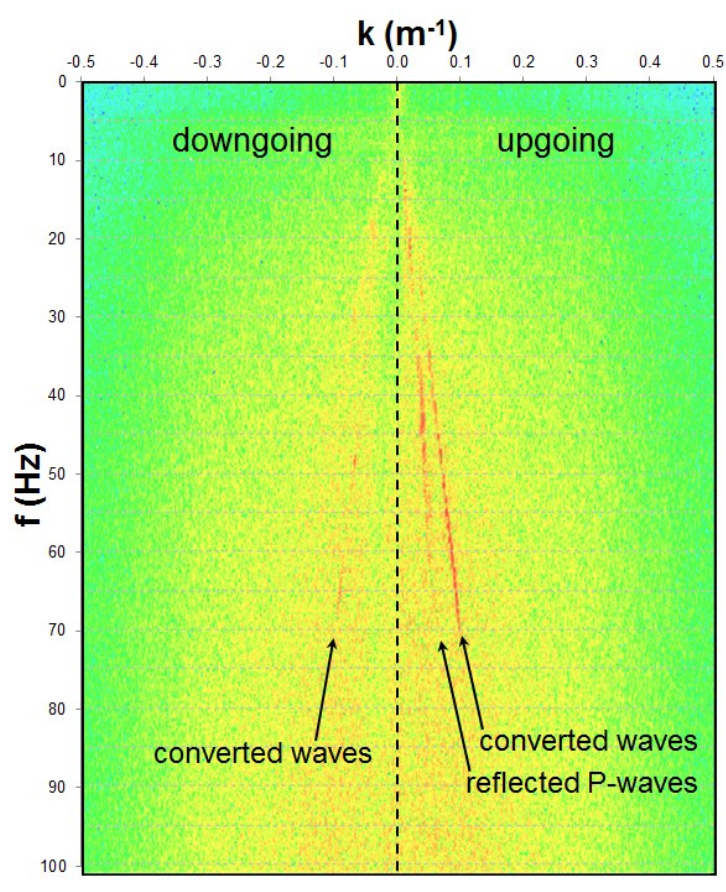

Figure 6-8: f-k spectrum of shot gather 46323812 from the monitor survey. Direct wave has been removed.

The only data-dependent steps in the processing sequence were shot-based amplitude scaling, median filtering, and deconvolution of the upgoing wavefields. One of the advantages associated with VSP is that it allows for the direct measurement of the downgoing wavefield at each sensor. This permits the use of deterministic deconvolution, using operators designed on data with a S/N typically $20-40 \mathrm{~dB}$ higher than the weak upgoing events used for surface-based processing (Hardage, 2000). Due to the relatively low $\mathrm{S} / \mathrm{N}$ deep in the DAS line, shot-based deconvolution operators were designed primarily based on the upper $1000 \mathrm{~m}$ of data. Best results for spiking deconvolution were 
attained with a 200 ms operator length and 1\% whitening applied. A zero-phase 10-1555-65 Hz band-pass filter was applied immediately afterwards. These parameters were optimized for the baseline data set, and applied to both vintages. Less aggressive frequency filtering would have suited the monitor data, but, in the interest of repeatability, filter characteristics were chosen as was appropriate for the noisier baseline data. The possibility of employing deconvolution operators derived from the less noisy downgoing monitor wavefield on the upgoing baseline wavefield was considered. However, it was largely ineffective because the source characteristics were too variable between vintages.

A 17 layer 1-D velocity model with 2-parameter anisotropy (Thomsen parameters $\varepsilon$ and $\delta$ (Thomsen, 1986)) was used to raytrace and migrate the data sets. The model was based on that used in Miller et al. (2016) and modified with velocities from the pre- $\mathrm{CO}_{2}$ injection observation well sonic log (Figure 6-9). The model was constructed by optimizing velocity and anisotropic parameters to match synthetic data to the DAS recordings. The volume was divided into horizontal $25 \times 25 \mathrm{~m}$ bins, and $2 \mathrm{~m}$ depth intervals for raytracing. Only P-wave imaging was performed due to the limited orthogonal sensitivity associated with the DAS system. The migration algorithm did not have an incorporated raypath-dependent scaling computation (Yilmaz, 1987), so shotbased inter-survey amplitude equalization was performed beforehand. The horizontal bin size was further reduced to $12.5 \times 12.5 \mathrm{~m}$ for migration. During raytracing and migration, a subject of concern was whether it was best to use the same raytracing results for both vintages despite the shifted shot locations between surveys, or to use separate raytracing results for each survey geometry. The concern with the latter option was that, if reflection 
points between surveys were only separated by a small distance, this shift could be exaggerated by traces being placed in different bin centers. If this proved to significantly reduce repeatability, then the solution would be to decrease bin size at the expense of computation time. Fortunately, the bin size did not require further infill, and separately raytracing each survey geometry proved effective. The migration used was a Kirchhoff integral-based algorithm (Schneider, 1978; Dillon, 1988). The algorithm did not include a phase and amplitude correction (i.e. a rho-filter), so the output was differentiated with respect to depth, and a $50^{\circ}$ phase rotation was further applied to match the zero-phase surface images.

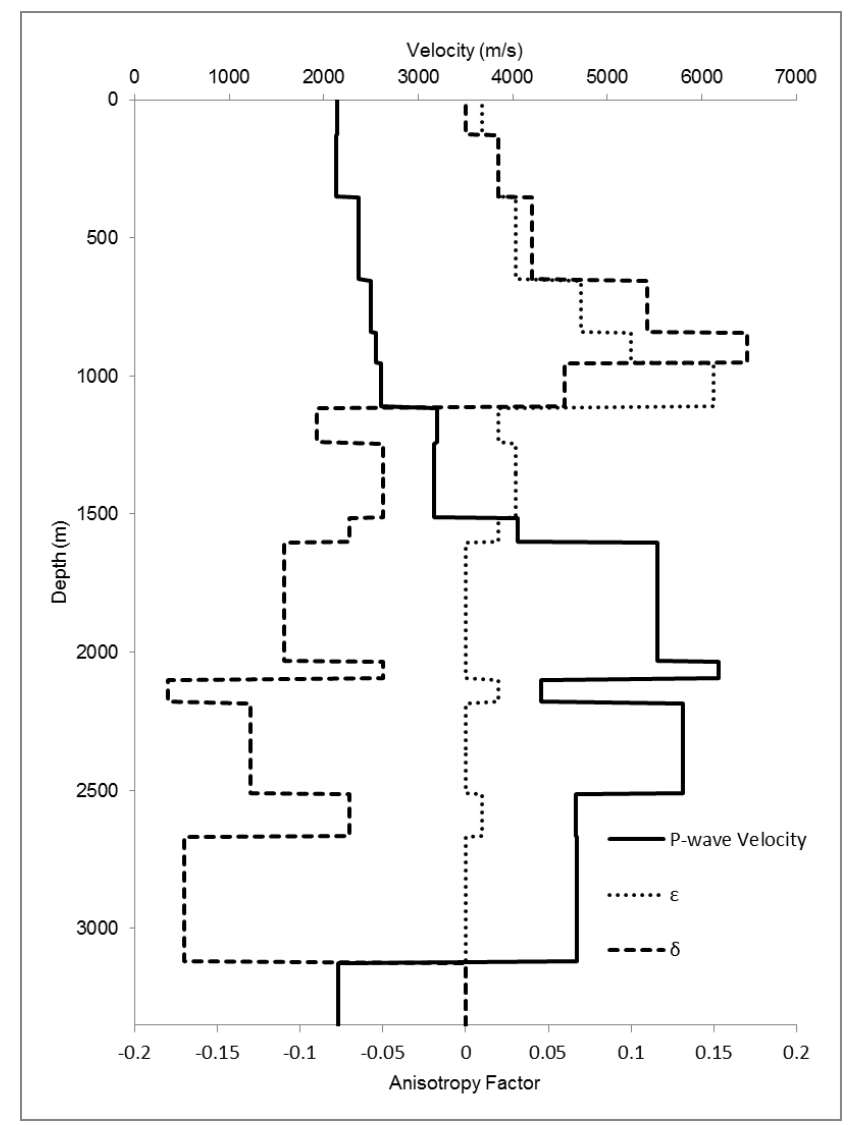

Figure 6-9: Anisotropic P-wave velocity model used for migration. 
One of the consequences of VSP geometry is that, unlike for the case of a surfacebased survey, the zone of specular illumination necessarily contains a blind-spot (Figure 6-10). The limits of this blind-spot in a vertical well are constrained by the nearest-offset shot and the deepest sensor in the array (Hardage, 2000). In our case, the fiber is not functional below $\sim 2800 \mathrm{~m}$ and the nearest-offset shot that is suitable for time-lapse analysis is $\sim 150$ m away from the observation well. The resulting specular blind-spot includes the entire location that the $\mathrm{CO}_{2}$ was expected to occupy at the time of the monitor survey (Harris et al., 2016). In order to image the reservoir in the immediate vicinity of the observation well with sufficient fold, the dip-limited migration aperture was set to $\pm 15^{\circ}$. Figure 6-11 compares the coverage at the reservoir level achieved by narrow aperture $\left( \pm 2.5^{\circ}\right)$ and wide aperture $\left( \pm 15^{\circ}\right)$ depth migration. Note the gap in the narrow aperture coverage below the observation well. Additionally, data from the uppermost $1000 \mathrm{~m}$ was not included in the final processing flow. Though these data had the highest associated $\mathrm{S} / \mathrm{N}$ values and could be used to image the shallow intervals quite well, their inclusion actually degraded the interpreted time-lapse difference close to the wellbore at the reservoir (Figure 6-12). As the plume migrates laterally, these uppermost sensors are expected to provide useful high $\mathrm{S} / \mathrm{N}$ information of the plume boundaries in future surveys. Prior to stacking, the prominent Prairie Evaporite horizon $(\sim 2500 \mathrm{~m})$ was picked and used to compute shot-based stack-power maximization static corrections. Refraction shot statics were also computed from the surface seismic data, but proved to be ineffective when applied to the VSP data. 


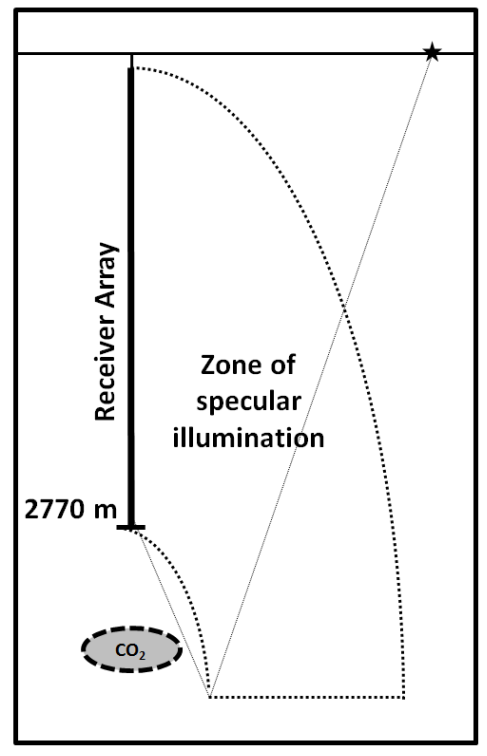

Figure 6-10: The expected position of the $\mathrm{CO}_{2}$ plume in relation to the 4D VSP zone of specular illumination necessitates the use of a wide imaging aperture. Grey line indicates the raypath from the closest shot recorded at the deepest sensor.
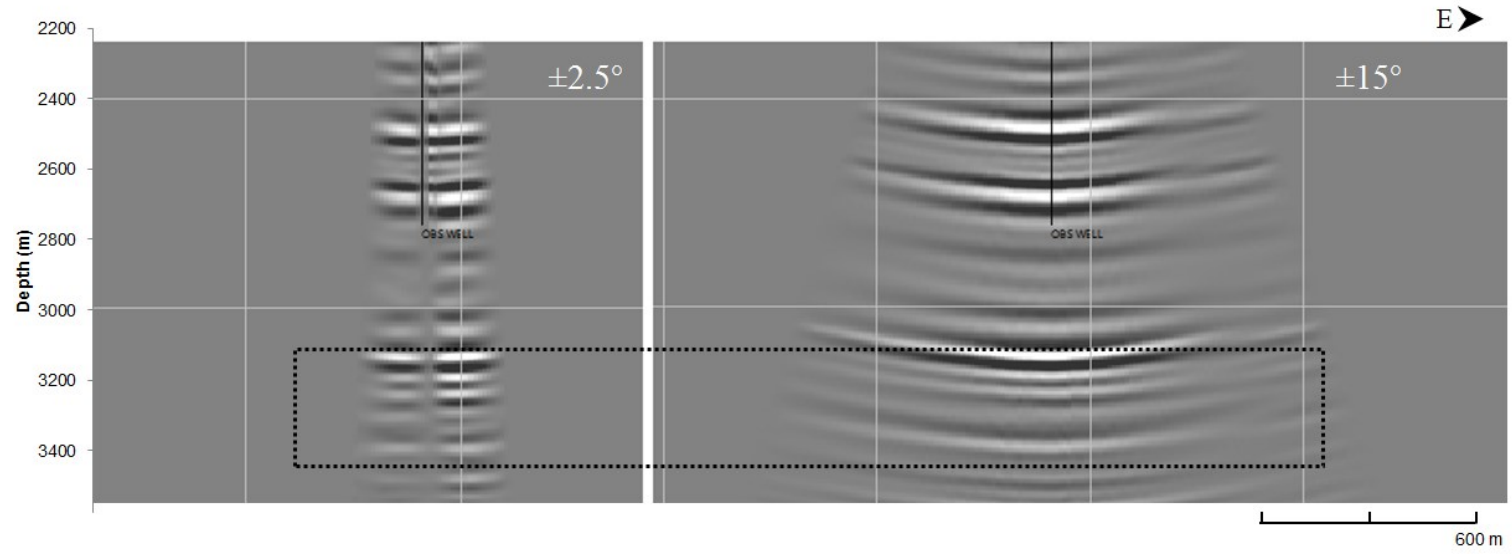

Figure 6-11: Stacks of the 9 monitor shot gathers that are closest to the observation well, depth migrated with a narrow aperture (left) or a wide aperture (right). Reservoir is enclosed by the rectangle delineated by a dashed line. 


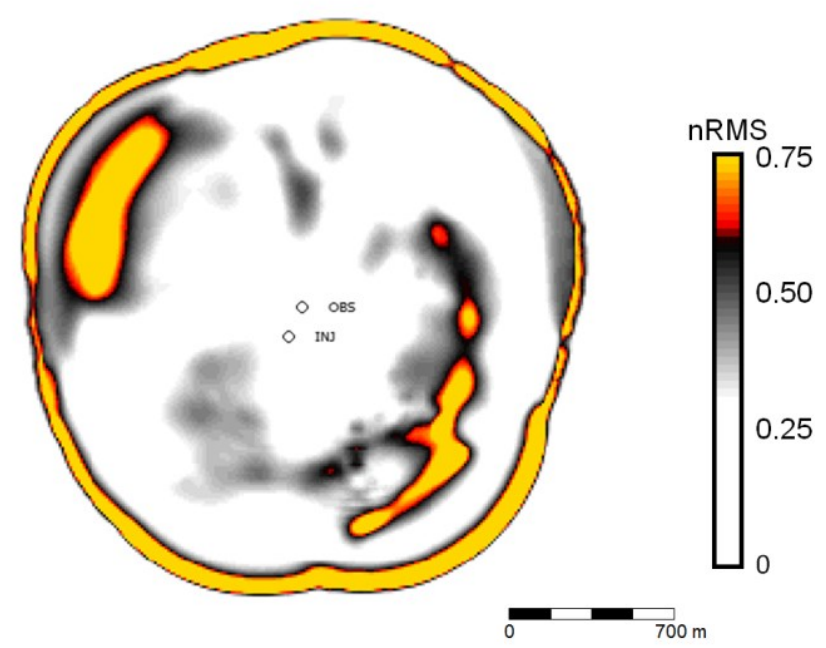

Figure 6-12: Plan view of the nRMS difference image for a horizon within the upper Deadwood interval of the reservoir. The values shown represent the mean nRMS values within a $20 \mathrm{~m}$ thick window centered on the horizon depth ( $\sim 3275 \mathrm{~m})$. Only data from the uppermost $1000 \mathrm{~m}$ of the DAS fiber was used.

Before time-lapse difference images could be constructed from the migrated volumes, they underwent poststack cross-equalization. This included trace-by-trace phase/time alignment, application of wavelet shaping filters, and amplitude equalization. In each case, operators were designed by analyzing a $2500-3100 \mathrm{~m}$ depth window to avoid cross-equalizing the reservoir zone. Phase/time shifts were applied using a minimum correlation threshold of 0.8 , and resulted in average time and phase shifts of $0.2 \mathrm{~ms}$, and $-0.5^{\circ}$, respectively. Cross-correlations of respective traces in both vintages were used to derive wavelet shaping filters. This technique attempts to match both frequency and phase spectra. A minimum correlation threshold of 0.7 was employed when applying these filters. Trace-by-trace RMS factors were computed to equalize monitor amplitudes to the baseline. The average scaling factor between vintages was found to be 1.03. Phase misalignment and wavelet variability were mitigated to an extent 
during prestack deconvolution, and amplitude scaling was addressed previously in the prestack sequence as well. The poststack cross-equalization step served to fine-tune the images and further improve repeatability (Li et al., 2011; Nguyen et al., 2015).

Further shot selection resulted in a total of 50 shot gathers comprising 44700 traces for migration. This subset included only shots located within $1000 \mathrm{~m}$ of the observation well, as predictions indicate the plume would be completely confined within a 500 m radius (Harris et al., 2016). Shot gathers were also omitted if their postprocessing difference gathers still exhibited strong upgoing energy, indicating poor repeatability. Figure 6-13 (left) shows the CDP fold at the top of the reservoir for the 50 shots included in the migration. Gaps in coverage are particularly large north and southeast of the observation well. The rectangular shot grid and spatially dense DAS line resulted in folds of 1-500 near the observation well. The fold map for a $\pm 15^{\circ}$ aperture Kirchhoff migration in Figure 6-13 (right) demonstrates that these coverage gaps can be closed for this 50 -shot subset, with $\sim 60000$ samples in bins between the two wells. 


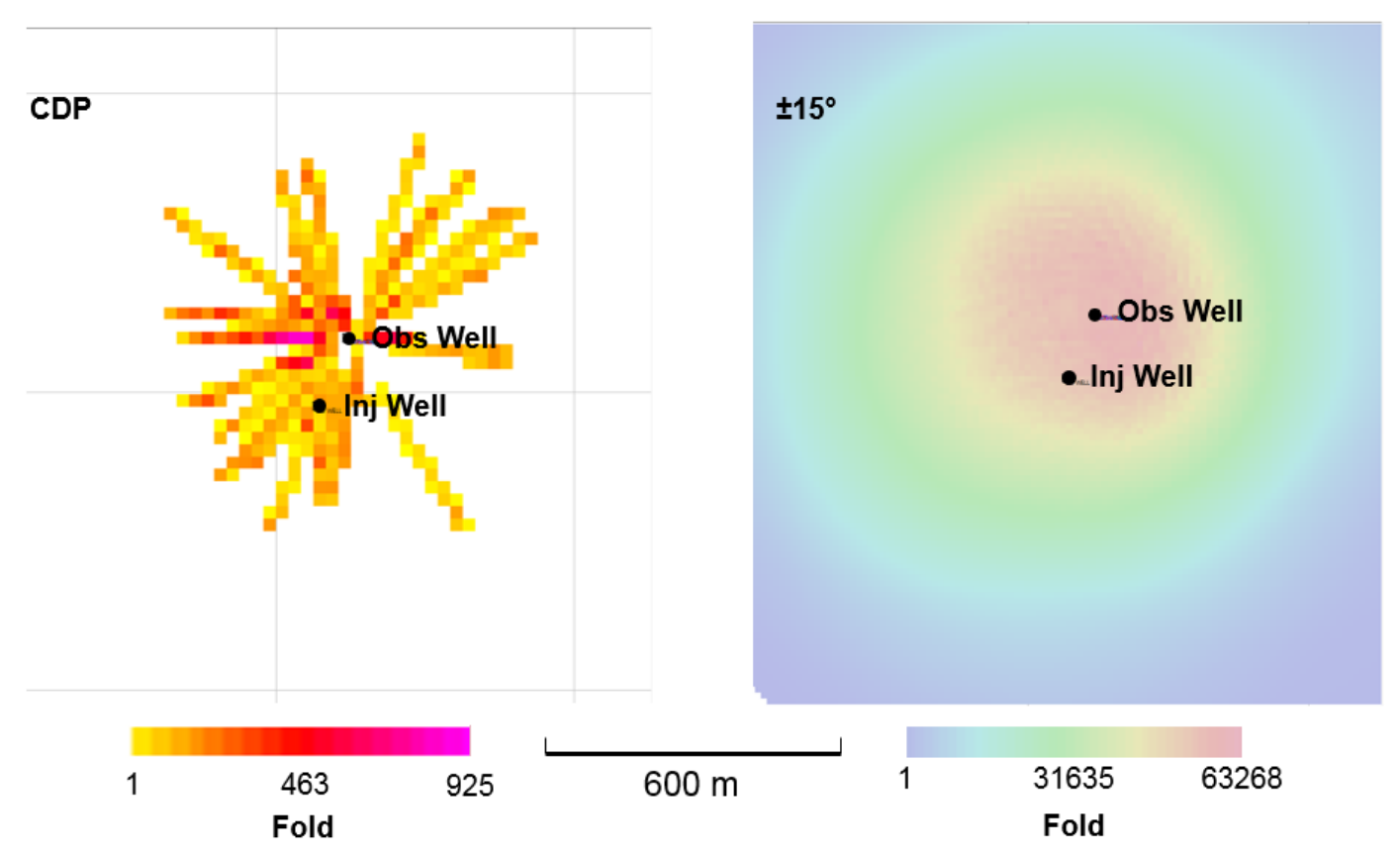

Figure 6-13: Fold map for the 50 shots included in the migration at reservoir top for specular reflections (left) and Kirchhoff migration with $\pm 15^{\circ}$ aperture (right).

\subsection{Results}

Figure 6-14 shows an example of a baseline, monitor, and difference gather in an early stage of the processing flow. At this stage, the difference gather is dominated by variations in the direct $\mathrm{P}$-wave, a converted wave that begins at $1000 \mathrm{~m}$, and additional low-velocity converted waves extending to $\sim 300 \mathrm{~m}$. The noise floor is limited by the low $\mathrm{S} / \mathrm{N}$ of the baseline data. These features are visible in almost all shot gathers. It is believed that the converted energy at $1000 \mathrm{~m}$ is a borehole guided wave (tube wave) induced by the change in cement quality that corresponds with this depth. The black arrows in the monitor gather indicate reservoir reflections that are beneath the noise floor in the baseline gather. Since the $\mathrm{S} / \mathrm{N}$ in the difference gather is limited by that of the baseline, it was not possible to observe the degree to which the reservoir residual was 
being reduced throughout processing. Thus shallower reflections had to be referenced for visual quality control.

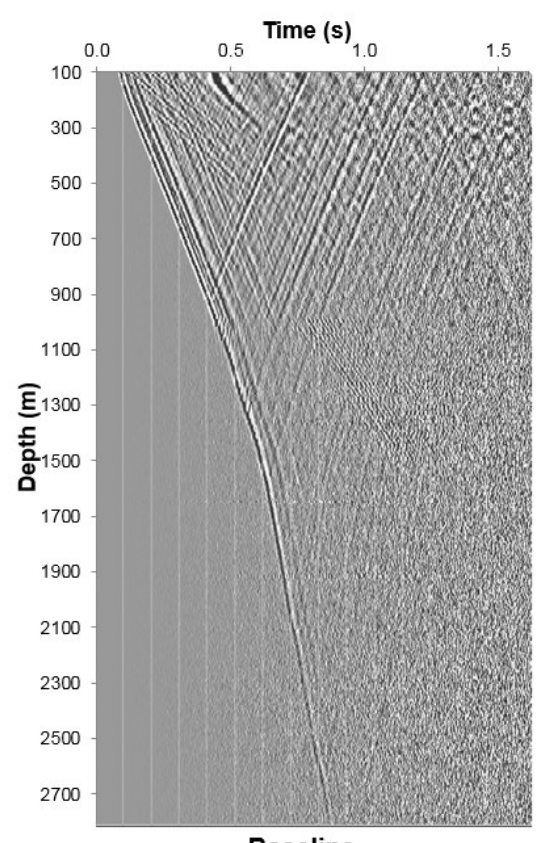

Baseline

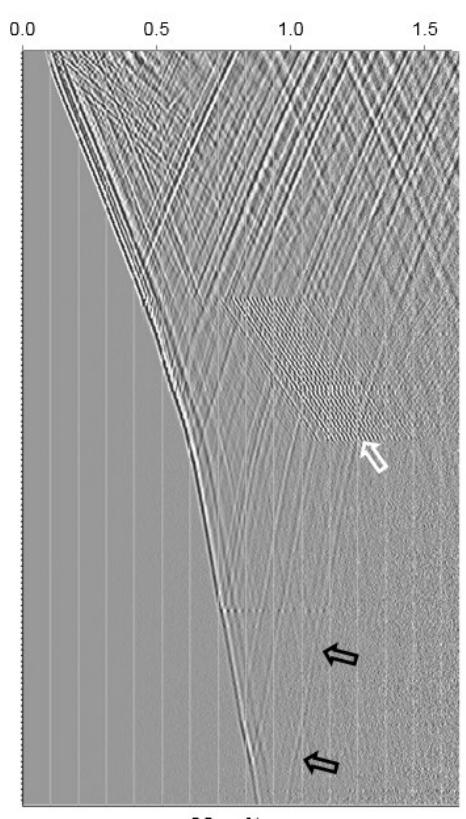

Monitor

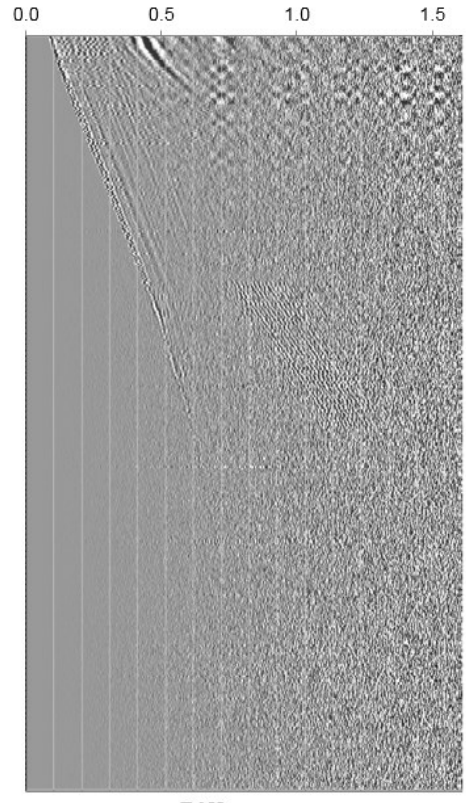

Difference

Figure 6-14: Baseline, monitor, and difference of shot gather 46323812 (150 m offset) after removal of near-surface traces, application of a Butterworth filter (10-80 Hz, $6 \mathrm{~dB}$ roll-off), $\mathrm{t}^{2}$ amplitude scaling, depth alignment, and amplitude cross-equalization. The difference gather is dominated by downgoing energy, tube waves, and random noise. Black arrows in the monitor gather indicate reservoir-based reflections that are not visible in the baseline data, and the white arrow indicates converted energy at $1000 \mathrm{~m}$. Baseline, monitor, and difference data are scaled by the same shot-based factor for display.

Difference gathers at three different stages of the pre-migration processing sequence are presented in Figure 6-15. Gather A shows an effective employment of the 51-trace median filter and subtraction to remove the direct P-wave, and gather B shows the result of a series of $f-k$ filters to remove the $\mathrm{S}$ - and tube wave energy. The upgoing wavefield in both A and B is faintly visible above the noise, which is typical of the shot 
gathers used in the imaging sequence. Gather $\mathrm{C}$ shows the difference between the deconvolved monitor and baseline gathers. In most shot gathers, the application of a deconvolution operator neither significantly improved nor diminished the repeatability of the data. This step was more beneficial to the quality of the migrated images, as it aided in the reconciliation of variable wavelets on a shot-by-shot basis.

Post-migration cross-equalization was applied to further improve repeatability. Histograms and cross-sections of nRMS values of the time-lapse differences (Figure 6-16 and Figure 6-17 respectively) show a small improvement in repeatability, as is evident in the increase of samples in the nRMS range $<0.4$ of the histogram. However, this processing step was unable to improve the repeatability of the poorest locations in the volume, as shown by the incomplete removal of high nRMS values near the eastern- and western-most areas of the cross-sections. The strong peak at a nRMS value of 2 in the post-processing histogram is due to the inability of the shaping filter to match spectra along the edge of the image. 


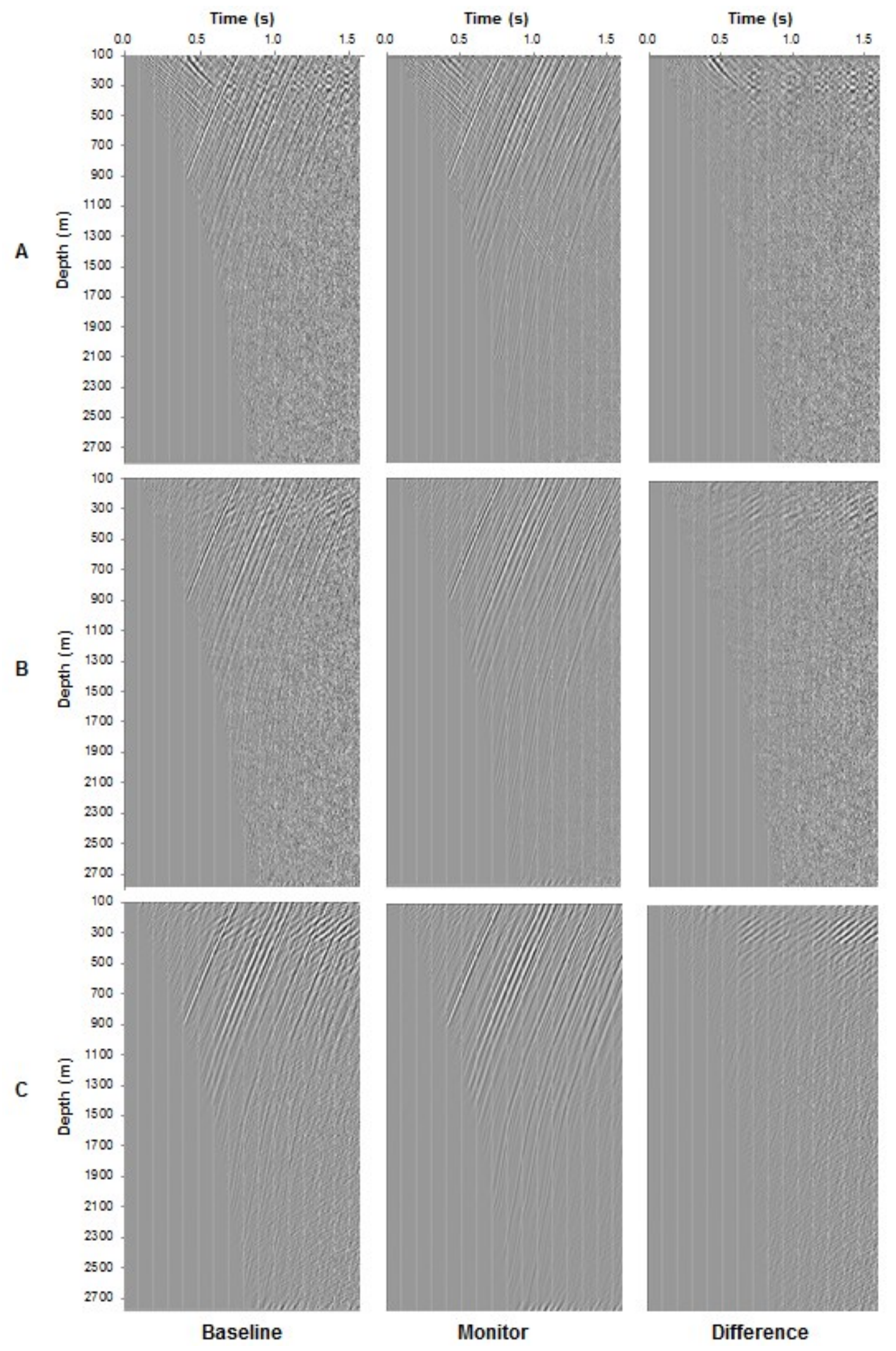

Figure 6-15: (caption next page) 
Figure 6-15: Baseline, monitor and difference shot gathers 46323812 at progressive stages in the processing sequence. Gather A shows difference after direct wave removal via median filter. Gather B shows difference after removal of remaining converted and tube waves via $\mathrm{f}-\mathrm{k}$ filtering. Gather C shows difference after shot-based deterministic deconvolution. Difference data are scaled by the same shot-based factor for display.
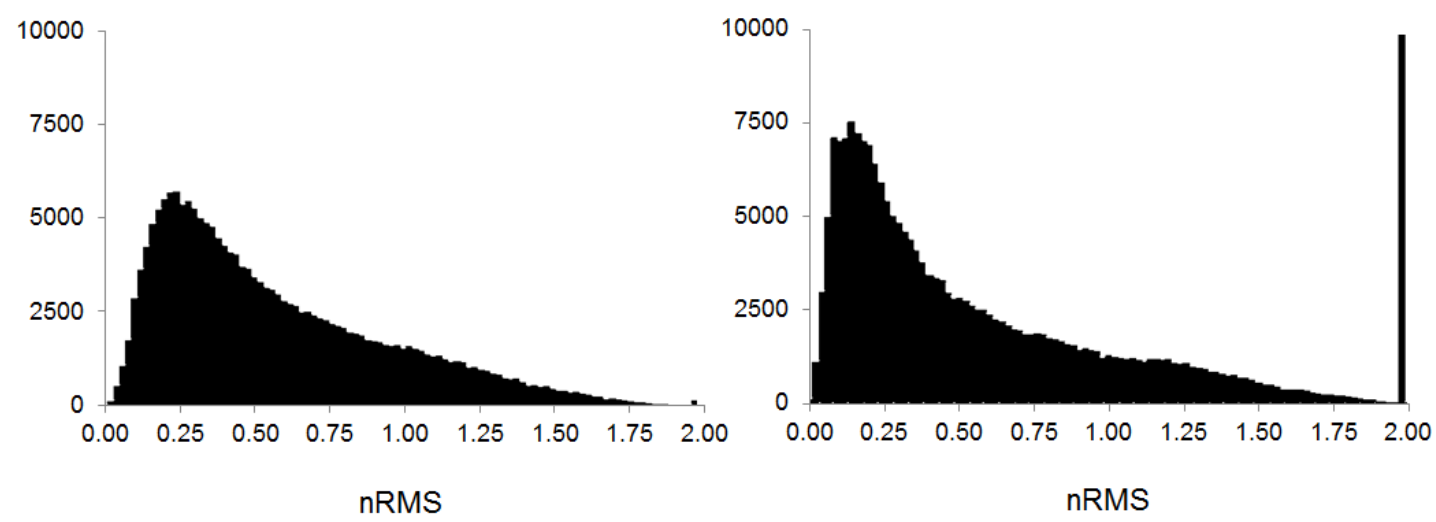

Figure 6-16: Histograms of nRMS values for time-lapse difference images before (left) and after (right) post-migration cross-equalization. Histograms include a subsurface volume extending $750 \mathrm{~m}$ from the injection well, and spanning a depth of $500-3000 \mathrm{~m}$ to avoid the effects of the near surface, basement, and potential $\mathrm{CO}_{2}$ plumes. Cross-equalization resulted in a global nRMS decrease from 0.44 to 0.37 . 


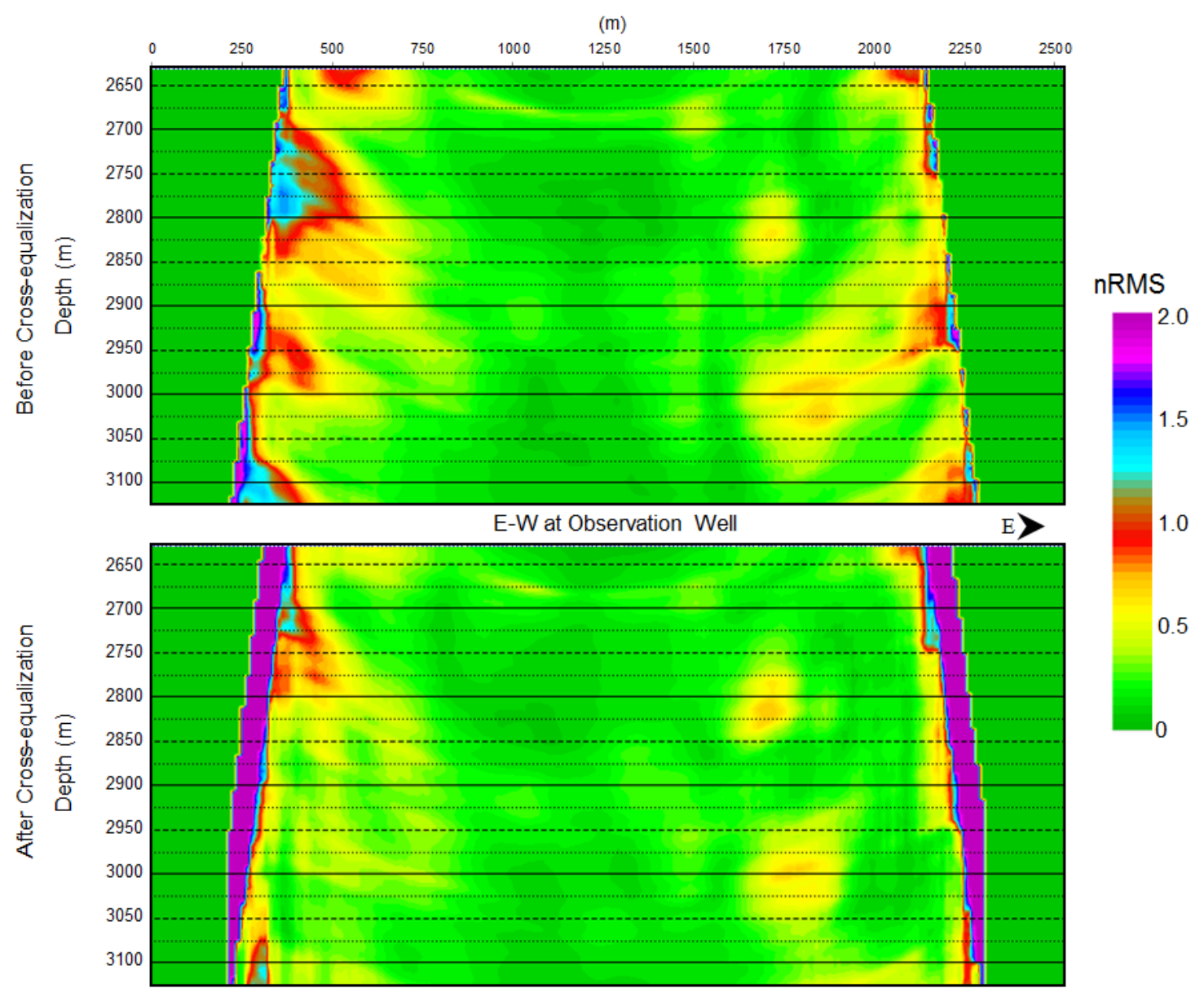

Figure 6-17: nRMS cross-sections immediately above reservoir depth before (top) and after (bottom) post-migration cross-equalization.

Figure 6-18 shows an E-W amplitude cross-section from the migrated baseline, monitor, and difference volumes. The images are nearly identical, and demonstrate the ability of the workflow to process and migrate the data in a repeatable fashion. To investigate the degree to which time-lapse differences may be detected and characterized, nRMS images are presented Figure 6-19. Values of nRMS are particularly high within the Precambrian basement where reflectivity is low and along the edges of the image. However, nRMS values are generally below 0.4 within $300 \mathrm{~m}$ of the observation well, which is comparable to surface surveys with good repeatability. The only anomalously 
high nRMS values in the proximity of the boreholes reside immediately below the

Deadwood C (depth $\sim 3275 \mathrm{~m}$ ), west of the observation well (left) and across the injection well (right). This anomaly is associated with nRMS values of up to 0.7 which is comparable to modeling results (Harris et al., 2016) where maximum nRMS values of $\sim 1.0$ were observed.

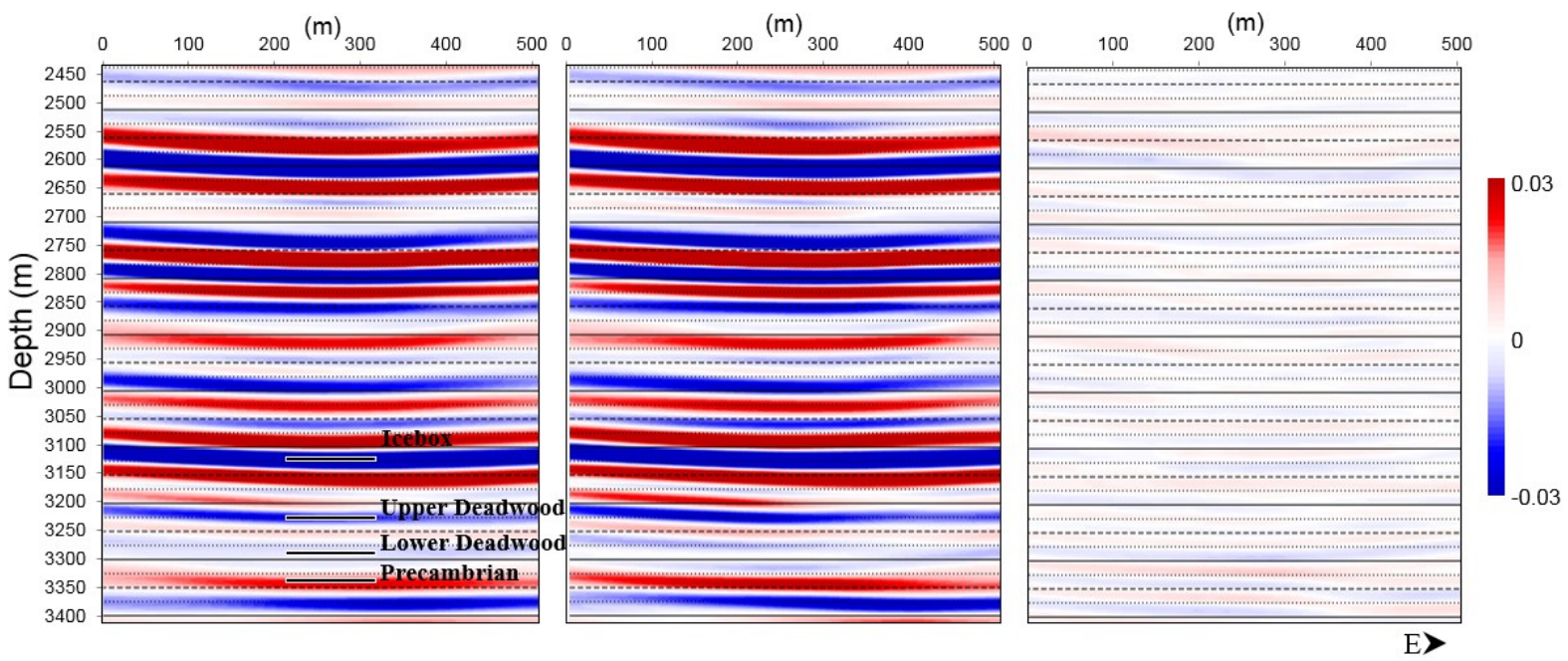

Figure 6-18: Amplitude cross-section of baseline (left), monitor (center), and difference (right) depth-migrated volumes intersecting the observation well. Key reservoir formations labeled on left. 


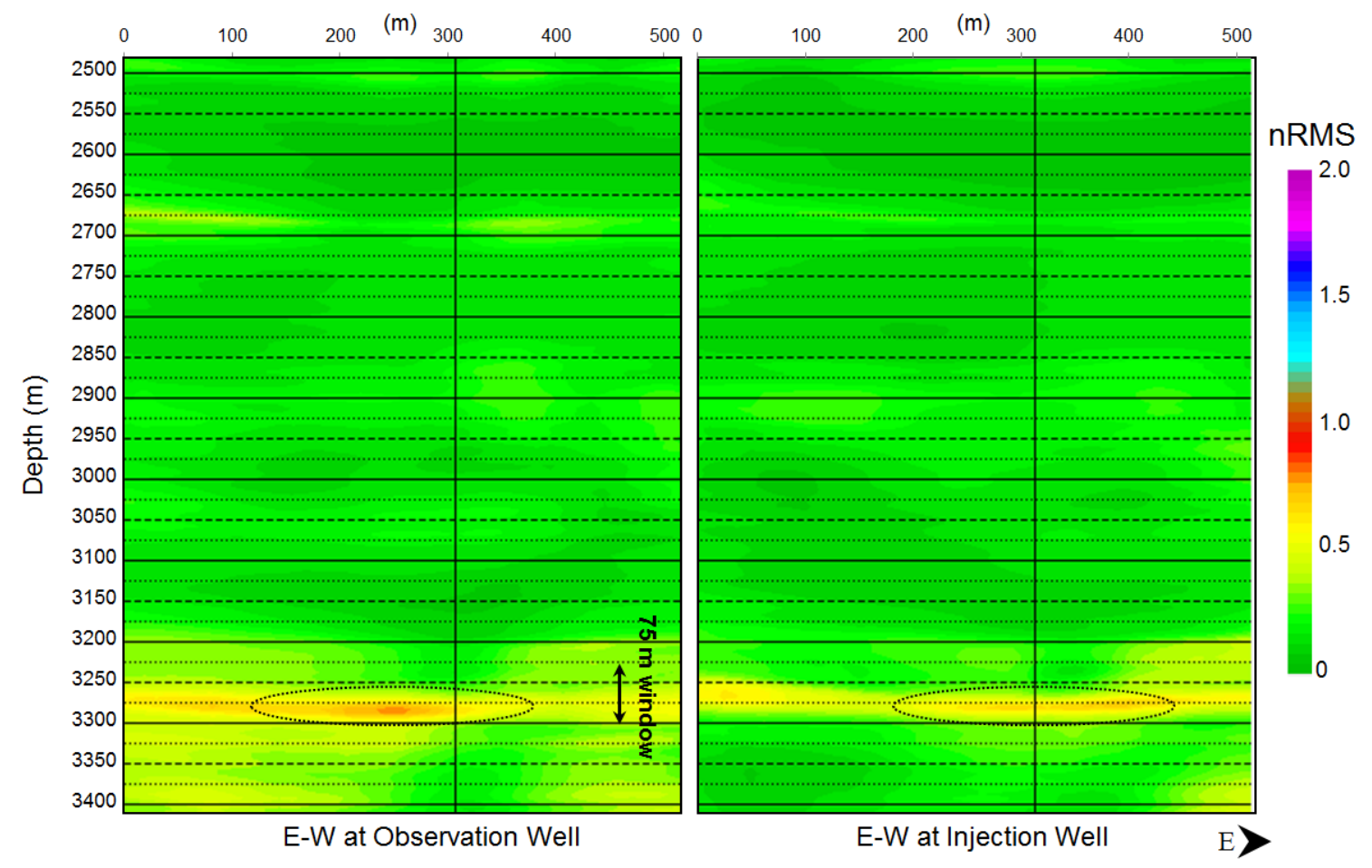

Figure 6-19: Values of nRMS of monitor-baseline differences for a $75 \mathrm{~m}$ window in crosssections through observation (left) and injection (right) wells. In both difference sections, a $\mathrm{nRMS} \approx 0.9$ anomaly is present at a depth of $\sim 3275 \mathrm{~m}$ (in dashed circle).

Figure 6-20 shows horizontal slices from the nRMS difference volume taken at depths corresponding to the caprock and the 3 injection intervals within the reservoir. From these images it is evident that: (1) Within a $\sim 500 \mathrm{~m}$ radius of the observation well nRMS values are generally below 0.25 , indicating good repeatability. (2) No significant nRMS amplitude differences are visible in the vicinity of the wells at either the Icebox cap rock or Black Island levels. (3) A nRMS anomaly $>0.7$ trends north-northwest from the injection well in the upper Deadwood Formation and diminishes in amplitude at the location of the observation well. Lower amplitude anomalies are present further from the well locations with no discernable pattern. (4) Low amplitude nRMS differences are visible in the lower Deadwood Formation in the vicinity of the wells and further from the 
well locations with no discernable pattern. In each zone, high nRMS values $(>0.75)$ characterize the edges of the imaged volume. These are due to the fact that these areas are of relatively low fold; thus random noise contributes more significantly to the difference images.

\subsection{Discussion}

Apart from the very high nRMS zones $(>0.75)$ in Figure 6-20 which are an artefact of low fold coverage near the edges of the images (particularly southeast of the wells), there are other high nRMS zones that reside at $>300 \mathrm{~m}$ from the observation well (visible as black in Figure 6-20). An example is shown in the cross-section in Figure 6-21. These zones are interpreted to correspond to low amplitude areas in the migrated images. When an nRMS volume is computed, any time-lapse differences in these zones are exaggerated by normalizing them with small amplitude values. Upon calculating an un-normalized RMS of the time-lapse differences, only one anomaly near the borehole remains. However, this anomaly appears to extend deeper in the RMS image than in the nRMS image, as it is not normalised by the comparatively large baseline and monitor seismic amplitudes (black trace in Figure 6-21). The near-borehole nRMS anomaly resides between 3270 and $3290 \mathrm{~m}$ as visible in Figure 6-19 and Figure 6-20, and is associated with values in excess of 0.7 , while the RMS anomaly extends down to $3350 \mathrm{~m}$ (Figure 6-21). 

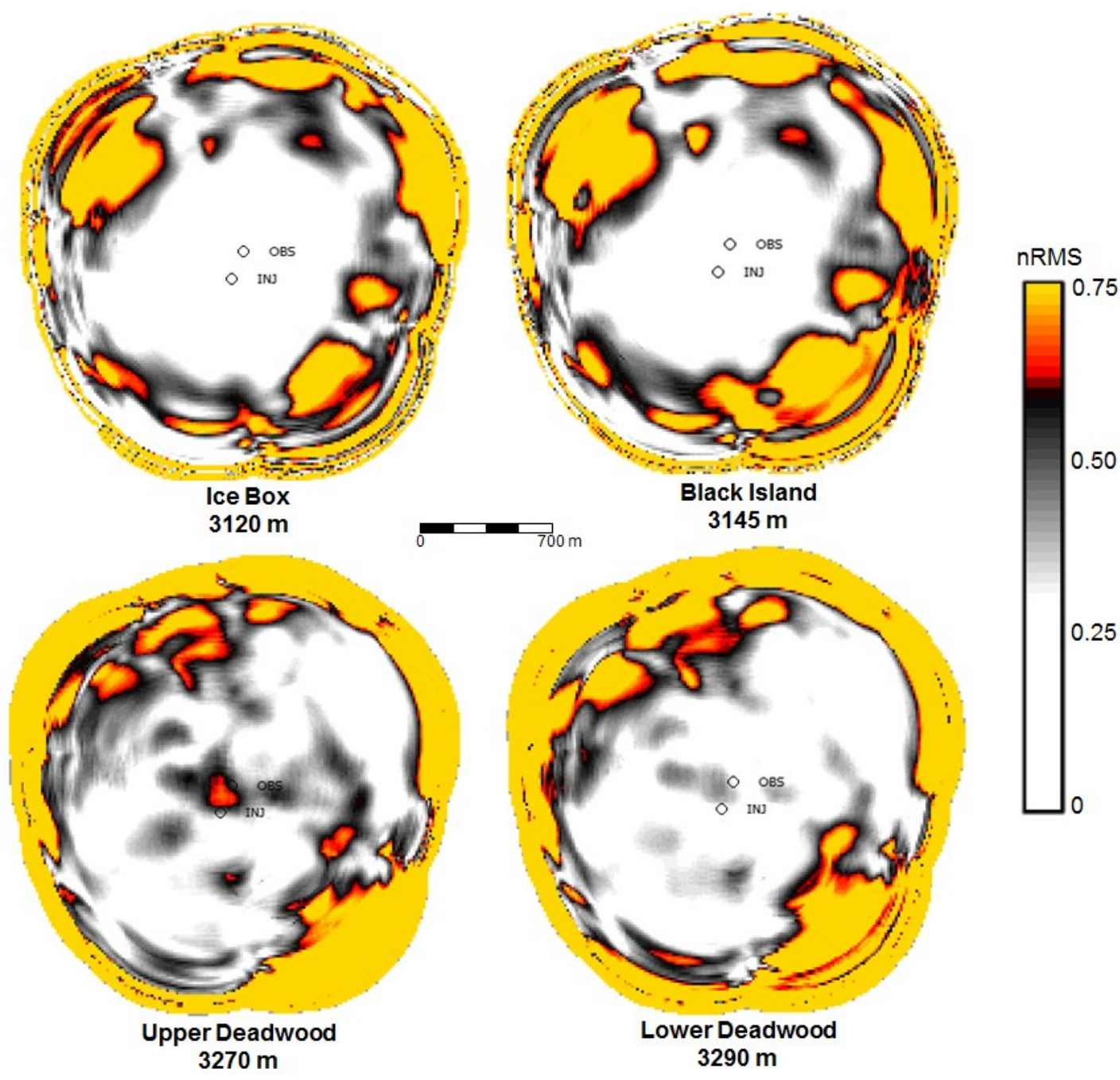

Figure 6-20: Plan view of nRMS difference images for the reservoir caprock (Ice Box formation) and 3 intervals where $\mathrm{CO}_{2}$ is injected. The values shown represent the mean nRMS values within a $20 \mathrm{~m}$ thick window centered on the horizon depth. The non-linear color scale is designed to exclude nRMS amplitudes less than 0.25 that are below the timelapse noise threshold and to emphasize amplitudes greater than 0.55 that are notably above the surrounding background levels. 


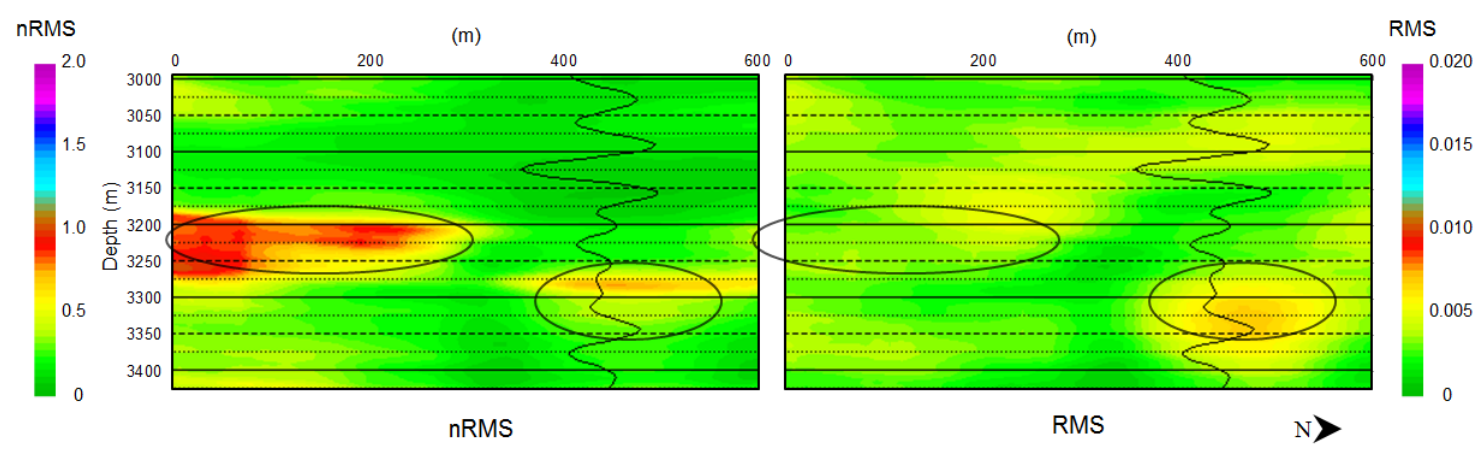

Figure 6-21: nRMS vs RMS comparison for a cross-section through the injection well computed with a $75 \mathrm{~m}$ window. A seismic amplitude trace from the baseline survey (black line) is superposed on the cross-section. Ellipses indicate anomalies resulting from a low amplitude zone (left), and possible $\mathrm{CO}_{2}$ plume (right).

The location of the nRMS anomaly corresponds to the upper Deadwood, which is the interval identified by Roach et al. $(2015,2017)$ as having the strongest seismic response to substitution of brine with $\mathrm{CO}_{2}$. Specifically, a decrease in seismic impedance in the upper Deadwood of $17 \%$, and $8-9 \%$ in the Black Island and lower Deadwood were predicted under these saturation conditions. In further support of these findings, Figure 6-22 shows a nRMS section of migrated synthetic DAS VSP data after 27 ktonnes of injected $\mathrm{CO}_{2}$ (modified from Harris et al. (2016)). The strongest plume-related nRMS values are located in the upper Deadwood (zone B), but the lower Deadwood (zone C) shows a considerable response as well. Despite the presence of a significant quantity of $\mathrm{CO}_{2}$ in the Black Island (zone A), the nRMS anomaly is weak even prior to the addition of any time-lapse noise. The study concluded that the plume will be visible in the Black Island in later stages of injection, but only under excellent repeatability conditions. It was also demonstrated that a small plume could be imaged in spite of the specular blind-spot. 
Although the blind-spot was somewhat smaller in Harris et al. (2016), the migration aperture was smaller as well $\left( \pm 6^{\circ}\right)$.

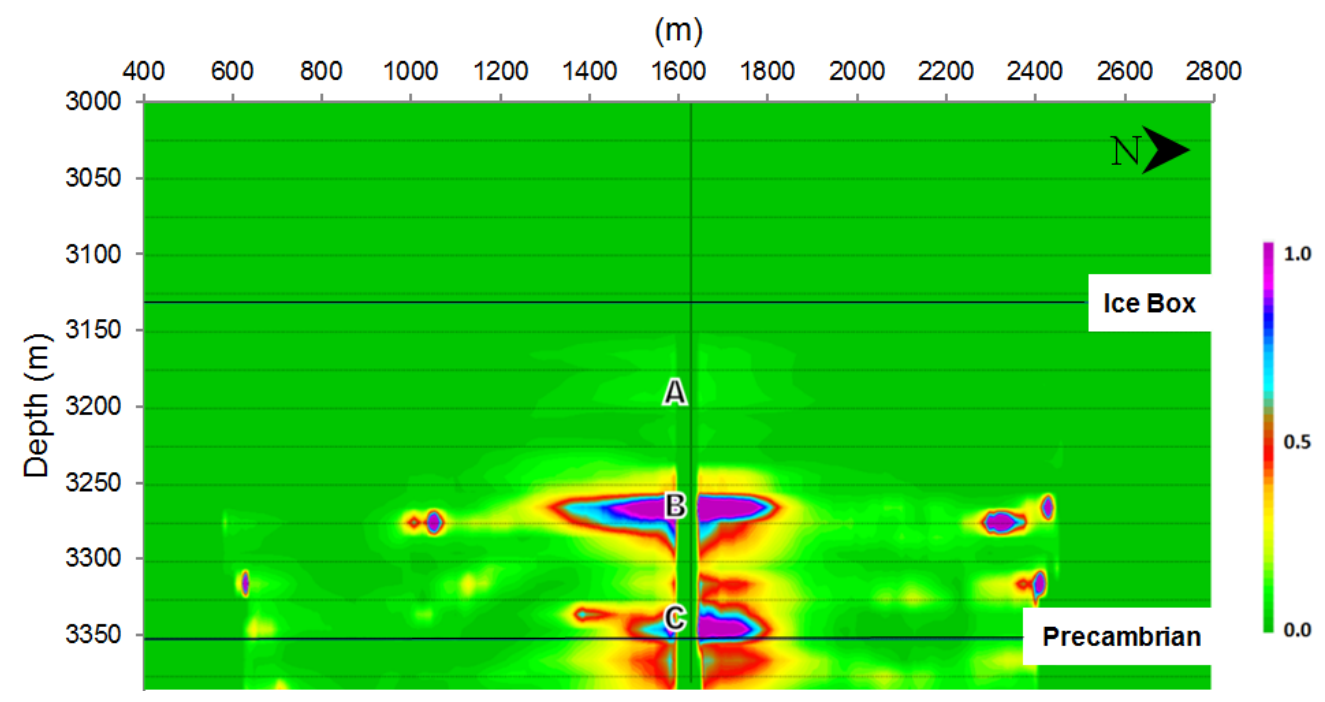

Figure 6-22: Cross-section of nRMS values for migrated synthetic data after 27 ktonnes of injected $\mathrm{CO}_{2}$. Labels $\mathrm{A}, \mathrm{B}$, and $\mathrm{C}$ correspond to the Black Island, upper Deadwood, and lower Deadwood formations, respectively. Black vertical line indicates the observation well.

At the time of the monitor survey, 36 ktonnes of $\mathrm{CO}_{2}$ had been injected and $\sim 90 \%$ of that volume was expected to be distributed equally into the upper and lower Deadwoods, with the remaining 10\% admitted into the Black Island based on spinner surveys. It is therefore likely that the anomaly presented in this study corresponds to $\mathrm{CO}_{2}$ located throughout the Deadwood. However, at this stage it cannot be quantified how the $\mathrm{CO}_{2}$ within the upper and lower parts of the Deadwood individually contribute to the total anomaly.

The identification of a plume-related anomaly is complicated by the fact that repeatability and sensitivity both likely vary with depth in the reservoir. The choice of an amplitude threshold for determining "anomalies" is subjective. Therefore it is necessary 
to identify zones of adequate repeatability and to refer to previous modeling results before attributing high time-lapse nRMS values to the presence of $\mathrm{CO}_{2}$. In Figure 6-20, the plume extends north of the observation well into a particularly noisy location. At minimum, the plume is bound by nRMS values of $\sim 0.55$, seen as dark grey in the map view. However, it is likely that the plume extends further north at lower saturations, as the shape continues at lower nRMS into the noisy areas.

Figure 6-23 shows a comparison of an nRMS depth window in the upper Deadwood Formation from the DAS VSP volume with an equivalent surface survey result (Roach et al., 2017). The background noise level is noticeably higher for the VSP image, likely due to the high noise floor in the VSP baseline data and the lack of near offset shots in the VSP grid. Otherwise, the size, shape, and location of the plumes are quite similar. The nRMS values of the anomaly are considerably higher in the VSP image. This can be largely attributed to differences in the size of the Fresnel zone at the reservoir depth for the two methods. For a zero-offset shot in the surface survey, the 50 $\mathrm{Hz}$ Fresnel radius is $\sim 350 \mathrm{~m}$ at the reservoir depth, which is significantly larger than the extent of the anomaly. For sensors at the bottom of the DAS array, the $50 \mathrm{~Hz}$ Fresnel radius is $\sim 100 \mathrm{~m}$, which is approximately the same size as the anomaly. The amplitude achieved after migration for a localized (i.e., sub-Fresnel zone) reflector will be a fraction of that expected for a comparable specular reflection, with the fraction being proportional to the size of the reflector relative to the Fresnel zone (e.g., Sheriff and Geldart, 1995). 

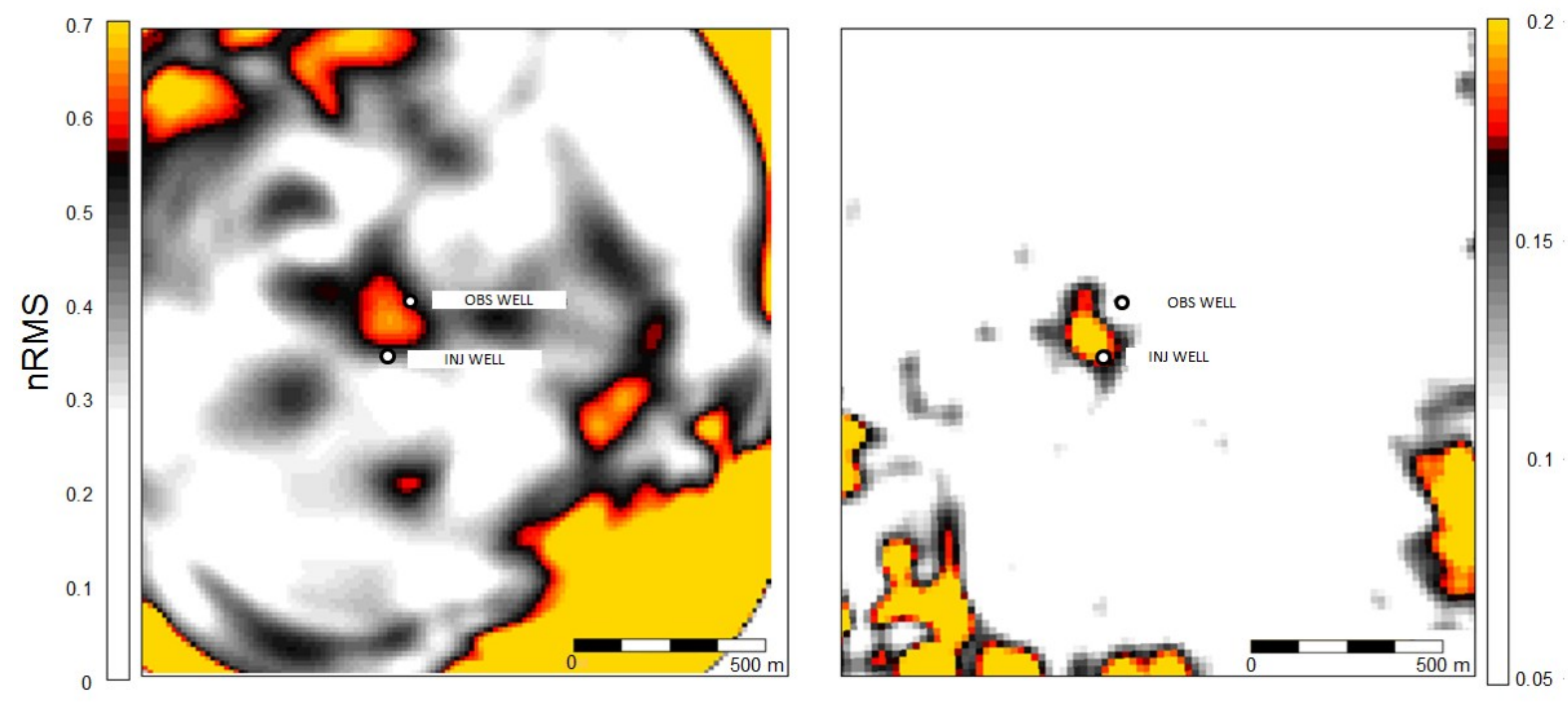

Figure 6-23: Plan view of nRMS difference images in the upper Deadwood showing VSP result (left) and surface-based result (right).

A significant limitation in the resolution of the difference images is the result of the narrow passband filter that was applied to the baseline data. As visible in Figure 6-4, the $\mathrm{S} / \mathrm{N}$ in the DAS (particularly for the baseline data) decreases significantly for the greater offset shot gather. A compromise is made when including the larger offset data in the images: a larger volume may be imaged, but bandwidth must be limited to ensure repeatability.

VSP is most effective for monitoring during the early stages of injection, if the injection and observation wells are in close proximity or collocated. This is evident when observing Figure 6-20: A high nRMS anomaly is present in the Deadwood in the vicinity of the wells. However, when using shot-receiver offsets less than $1000 \mathrm{~m}$, noise along the edge of the image exceeds an acceptable threshold $\sim 700 \mathrm{~m}$ from the observation well. The methodology developed in this study will be applied in the near future as the plume expands, enabled by the larger offset shot gathers available. However, repeatability tends 
to decrease in the larger offsets, and is generally limited by the noise floor in the baseline data. For forthcoming vintages, it may be more effective to utilize the first monitor survey in place of the baseline survey for $4 \mathrm{D}$ processing. This would be expected to improve repeatability for a few reasons: (1) The monitor and baseline data would exhibit similar $\mathrm{S} / \mathrm{N}$ characteristics, allowing trace-by-trace nRMS to be employed as a quality control metric after each processing step. (2) Processing parameters would not need to be compromised to suit data sets of two dissimilar $\mathrm{S} / \mathrm{N}$ characteristics, resulting in a suboptimal processing flow for each. (3) Repeatability would not be limited by the noise floor from the 2013 data, which was a consequence of a less sophisticated postprocessing sequence. Two issues would need to be addressed if the first monitor was to be used as the baseline: (1) It would be necessary to account for the signature of the plume at the time of the first monitor, and include it in the time-lapse difference of subsequent surveys. (2) There are no shot offsets $<150 \mathrm{~m}$ in the first monitor survey, so using it as a baseline for subsequent monitors would propagate the specular blind spot effect mentioned previously.

Many of the processing steps undertaken in this study can be considered to be portable in future 4D DAS VSP studies. However it should be stated which aspects of the workflow were required by characteristics specific to this dataset. Choices of thresholds for shot omission are expected to vary highly from study to study. There is a trade-off between coverage and repeatability when including more low-quality shots in the stack. Study areas with higher lateral variability in the uppermost subsurface will likely require a smaller threshold for inter-survey shot-location shifts. The shot-based amplitude scaling implemented in this study is also not necessarily the most effective for general 
applications. Windows centered on specific reflections rather than the direct wave may be more effective for amplitude cross-equalization or spectral matching in 4D datasets with more similar $\mathrm{S} / \mathrm{N}$ characteristics. The choice of migration aperture was also constrained by this specific dataset due to the location of the plume in a specular blind-spot. The use of a smaller aperture was not an option in this study, and it is possible that a different aperture width would have been more effective under a different survey geometry.

\subsection{Conclusions}

The results of time-lapse DAS VSP analysis from the Aquistore $\mathrm{CO}_{2}$ storage site have been presented following the processing of datasets from November 2013 and February 2016. These relatively early findings over the course of the project have shown DAS VSP to be an effective method for time-lapse monitoring of $\mathrm{CO}_{2}$ in a deep saline reservoir. This conclusion is supported by:

1) The acceptable repeatability attained at the reservoir level despite the variable $\mathrm{S} / \mathrm{N}$ characteristics associated with baseline and monitor vintages. Time-lapse noise did not exceed an nRMS of 0.4 within $\sim 300 \mathrm{~m}$ of the observation and injection wells. This repeatability is expected to further improve if the monitor data herein is utilised as the baseline for subsequent surveys. This is due to an anticipated lower noise floor, and the inclusion of more shot gathers in the final stacks.

2) A nRMS anomaly at $\sim 3270-3290 \mathrm{~m}$ with associated values in excess of 0.7 , and a RMS anomaly at $\sim 3270-3350$ m was observed extending from the injection well with a north-northwest trajectory in the Deadwood Formation. No significant residuals were observed in the Black Island, as saturation levels have not exceeded seismic detectability thresholds. Based on the findings of Harris et al. (2016), it is expected that a 
$\mathrm{CO}_{2}$-based anomaly will be visible in the Black Island only under excellent repeatability conditions.

\subsection{Acknowledgements}

The authors would like to thank the Petroleum Technology Research Centre and Kyle Worth in particular for project management, and SaskPower. Brian Roberts, Lisa Roach, Ivan Marsden and Michelle Robertson are thanked for their fieldwork at Aquistore. The seismic data were acquired by Silixa, Schlumberger Carbon Services, Tesla Exploration, Geospace Technologies and the Geological Survey of Canada, with help from Lawrence Berkeley National Laboratory and Thomas Daley. Funding for Lawrence Berkeley National Laboratory was provided through the Carbon Storage Program, U.S. DOE, Assistant Secretary for Fossil Energy, Office of Clean Coal and Carbon Management through the National Energy Technology Laboratory, of the U.S. Department of Energy, under contract No. DE-AC02-05CH11231. The migration velocity model was based on that produced by Douglas Miller of Miller Applied Science LLC under contract from Silixa Limited. We would also like to thank Chevron for their contributions. This is publication 20170175 of the Geological Survey of Canada. 


\section{4D Vertical Seismic Profile Modeling of $\mathrm{CO}_{2}$ Injection Scenarios at Aquistore}

\subsection{Abstract}

The Aquistore carbon capture and storage project (Saskatchewan, Canada) is currently employing $3 \mathrm{D}$ vertical seismic profiles to monitor $\mathrm{CO}_{2}$ injected in a geological reservoir at a depth of $>3200 \mathrm{~m}$. Distributed acoustic sensing has previously been shown to be an effective tool for imaging the $\mathrm{CO}_{2}$ plume at the research site, and this study presents a continued effort in characterizing its trajectory, location, and size via fluid flow simulations, fluid substitution calculations, and forward modeling of seismic data. Time-lapse synthetic seismic volumes corresponding to two injection quantities, 36 and $97 \mathrm{kt}$, are compared alongside field data with nearly identical acquisition geometries and processing flows. The modeling results support the interpretation, based on field data, that most of the $\mathrm{CO}_{2}$ signal originates from the lower Deadwood reservoir unit. However, it is apparent that more effort is required to predict the trajectory of the plume, and the seismic amplitude contrasts caused by fluid replacement.

\subsection{Introduction}

Monitoring the migration of injected $\mathrm{CO}_{2}$ is necessary in carbon capture and storage (CCS) projects to ensure containment to the reservoir. Seismic methods, including vertical seismic profiles (VSP), have been successfully employed to characterize $\mathrm{CO}_{2}$ plume geometry (e.g., Ahmadi and Morozov, 2011; Coueslan et al., 2013). A time-lapse VSP study is currently underway at the Aquistore CCS project (Saskatchewan, Canada) to characterize the $\mathrm{CO}_{2}$ plume during the early stages of 
injection. Injection commenced in April 2015, and was preceded by a baseline survey performed in November 2013. Two monitor VSP surveys have since been performed in February and November 2016. Under sustained injection rates of 400-600 t/day since Fall of 2015 (Worth et al., 2015), the monitor surveys corresponded to $36 \mathrm{kt}$ and $105 \mathrm{kt}$ of injected $\mathrm{CO}_{2}$, respectively. Interpretation of the February 2016 data regarding the geometry of the $\mathrm{CO}_{2}$ plume was presented in Harris et al. (2017). In this paper, we assess the validity of that interpretation by comparing field VSP data with fluid flow simulations. We also further assess the imaging conditions using a full synthetic seismic dataset calculated for the simulations. Finally, the flow simulation results are considered against the seismic imaging constraints.

In this study, a three-stage workflow is implemented to estimate the time-lapse seismic response due to the injection of $\mathrm{CO}_{2}$ at the Aquistore study site. First, fluid flow simulations were performed for injection scenarios corresponding to each monitor survey. These were "history-matched" simulations in the sense that highly variable injection rates (Figure 7-1), temperatures, injectivity, and field pressure responses were replicated in the models (EERC, 2016). Fluid-replacement calculations were then completed to estimate the effect of pore-space fluid substitution on the elastic properties of the reservoir. These calculations followed the formulations put forth in Gassmann (1951). Finally, 3D finite-difference time-domain (FDTD) elastic seismic simulations were run for each injection scenario, replicating the shot-receiver geometry from the field surveys. These synthetic data were processed and imaged using the same workflow as used for the field data. 


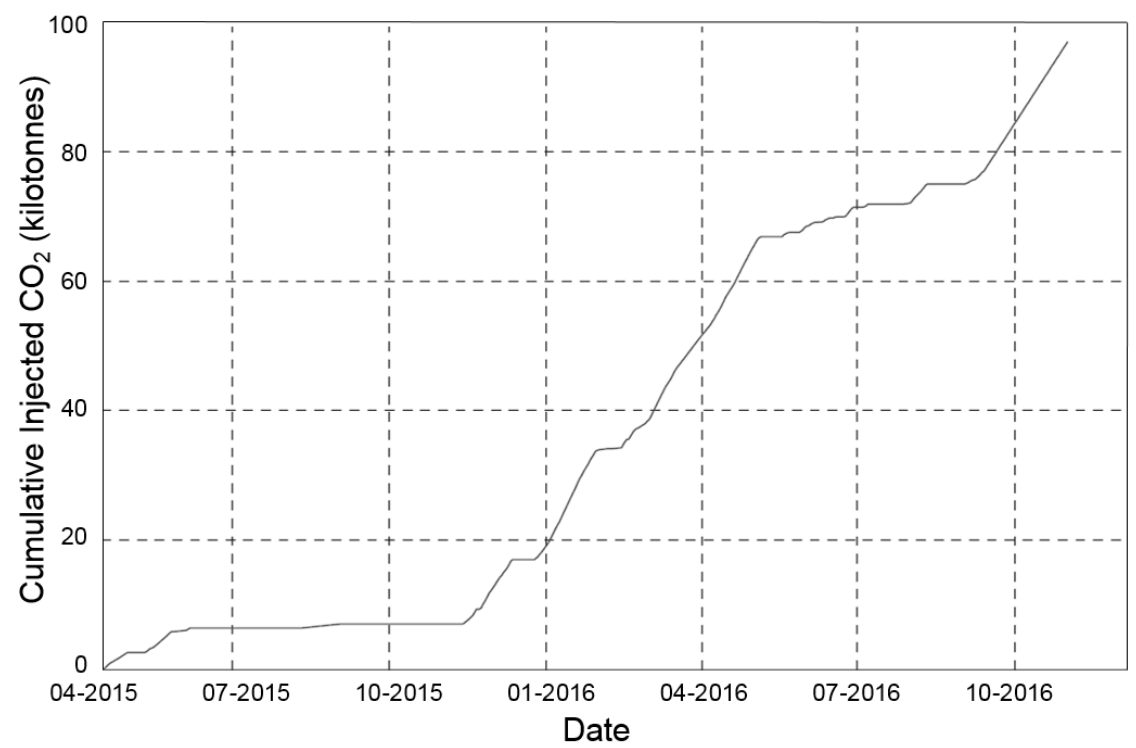

Figure 7-1: Curve of cumulative injected $\mathrm{CO}_{2}$ versus time used in fluid flow simulations.

An optical-fiber sensor system, distributed acoustic sensing (DAS), is deployed down an observation well located $150 \mathrm{~m}$ from the injection well. The field data presented here were recorded on the DAS system, and all synthetic seismic data presented here assume the same DAS line geometry.

\subsubsection{The Aquistore $\mathrm{CO}_{2}$ storage site}

The Aquistore storage site is located in southeastern Saskatchewan, Canada, near the town of Estevan (Figure 7-2). $\mathrm{CO}_{2}$ is captured from the coal-fired SaskPower Boundary Dam Power Station and transported via an underground pipeline to the injection well. The injection target is a $\sim 200 \mathrm{~m}$ thick sequence of sandstones separated by shale units at a depth interval of $3150-3350 \mathrm{~m}$. The reservoir is immediately underlain by the Precambrian basement, delineating the bottom of the Williston Basin (Figure 7-2). The $\sim 15 \mathrm{~m}$ thick Ice Box shale directly overlies the reservoir and constitutes the primary seal, while the $\sim 150 \mathrm{~m}$ thick Prairie Evaporite serves as a regional seal at a depth of 
$\sim 2500 \mathrm{~m}$. The injection well is perforated at 4 depths in the reservoir to permit the entry of $\mathrm{CO}_{2}$ : one perforation zone in the Black Island $(3175 \mathrm{~m})$, two perforation zone in the upper Deadwood (3230 and 3255 m), and one perforation zone in the lower Deadwood (3300 m).

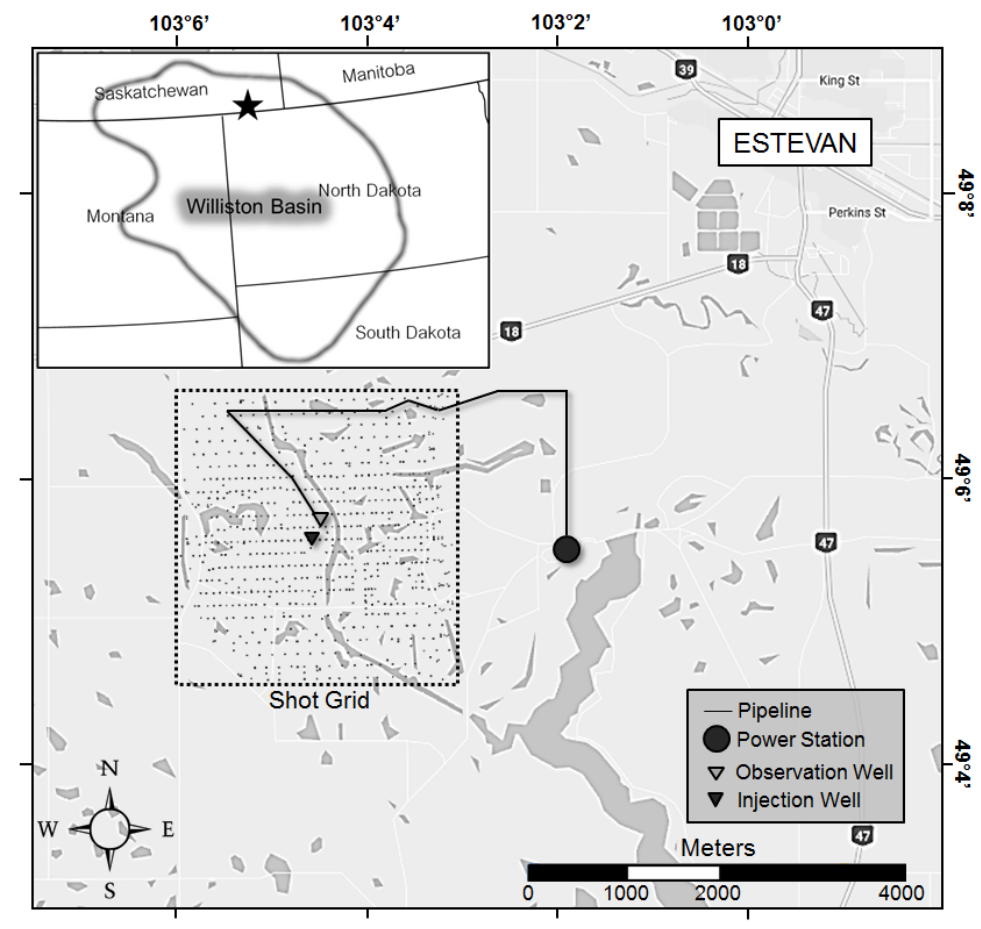

Figure 7-2: Location of Aquistore $\mathrm{CO}_{2}$ storage site (shown as black star in upper-left map) and seismic survey area (enclosed in dotted box).

\subsubsection{Previous studies}

Preliminary seismic modeling presented in Harris et al. (2016) established the feasibility of using DAS VSP to monitor the injected $\mathrm{CO}_{2}$ plume at Aquistore for a minimum quantity of $27 \mathrm{kt}$. This conclusion was based on fluid flow simulations conducted in advance of any $\mathrm{CO}_{2}$ injection at the site and were based on hypothetical injection scenarios. The feasibility criterion required that the seismic response due to the 
presence of the $\mathrm{CO}_{2}$ plume exceed the expected repeatability of the surveys. Seismic response and repeatability were both expressed as normalized root-mean-square (nRMS) of the time-lapse amplitude differences between subsequent active-source surveys. As only one field survey had been performed by that time, repeatability was estimated by analyzing migrated, cross-equalized shots that were in close proximity to each other. Prestack data was shown to have a repeatability of nRMS $<0.7$ at the reservoir depth, while seismic responses due to the presence of the plume yielded nRMS $>1$, and extended further than $200 \mathrm{~m}$ from the injection well. While Harris et al. (2016) was an encouraging proof of concept, there were a few limitations with those data that did not allow for accurate predictions of plume geometry throughout the injection timeline. The fluid flow simulations did not incorporate the actual injection activity achieved in the field, the seismic simulations used shot locations that did not represent the final field survey geometry, and the seismic processing and imaging was 2D.

Analysis of the first monitor survey was presented in Harris et al. (2017). The study demonstrated that a repeatability of nRMS $<0.4$ was achieved between the baseline and first monitor surveys within $\sim 300 \mathrm{~m}$ of the observation and injection wells. Additionally, a time-lapse anomaly in the reservoir associated with nRMS $>0.7$ was attributed to the presence of $\mathrm{CO}_{2}$. One of the factors that limited the sensitivity and vertical resolution was the relatively low signal-to-noise ratio in the baseline data. This necessitated the application of a narrow band-pass filter to both data vintages, and yielded a high noise-floor in the difference images. A consequence of the reduced vertical resolution was that the depth of the plume could not be precisely constrained. The seismic anomaly attributed to the presence of the $\mathrm{CO}_{2}$ plume was determined to reside 
within the Deadwood Formation, but could not be further localized into the upper or lower units of the formation.

\subsection{Methods}

A geological model of the reservoir was constructed by the EERC using nearby wells to assign various lithological properties to the different layers. This model was used in fluid flow simulations for various injection scenarios. The geological model and simulation results were then combined to construct seismic models for each injection scenario, including P- and S-wave velocities, and densities for each reservoir gridpoint. A description of each model construction and the subsequent seismic simulation is described in this section, as well as the seismic processing undertaken thereafter.

\subsubsection{Geological model and fluid flow simulations}

Logs from 15 wells in southern Saskatchewan and northern North Dakota were analyzed to determine shale volumes and total porosity, 9 of which penetrated the sedimentary sequence to the bedrock (EERC, 2014). Shale volumes were calculated from gamma-ray logs, and porosity from the neutron density logs. The shale volume values were used to stratify the reservoir model into 6 sandstone storage zones, each separated by shale units. The model was then filled by stochastically (Gaussian) populating shale volumes and total porosities using vertical and lateral variograms for each reservoir zone. Then, effective porosities were calculated using shale volumes and total porosity, and permeabilities were computed from porosities using an empirical relationship. The reservoir was also populated with temperature and pressure values in order to calculate $\mathrm{CO}_{2}$ densities in the dynamic models. This regional model was clipped to a $34 \mathrm{~km}^{2}$ area around the study site with $7.6 \times 7.6 \mathrm{~m}(25 \times 25 \mathrm{ft})$ grid spacing. To reduce computing 
time, the model was resampled to maintain high resolution around the injection and observation wells, but gridpoint spacing was increased to $229 \mathrm{~m}$ (750 ft) further from the wells, as visible in Figure 7-3. The volume is sampled irregularly in depth, with layer spacing ranging between $1.26-2.76 \mathrm{~m}$ (EERC, 2014).

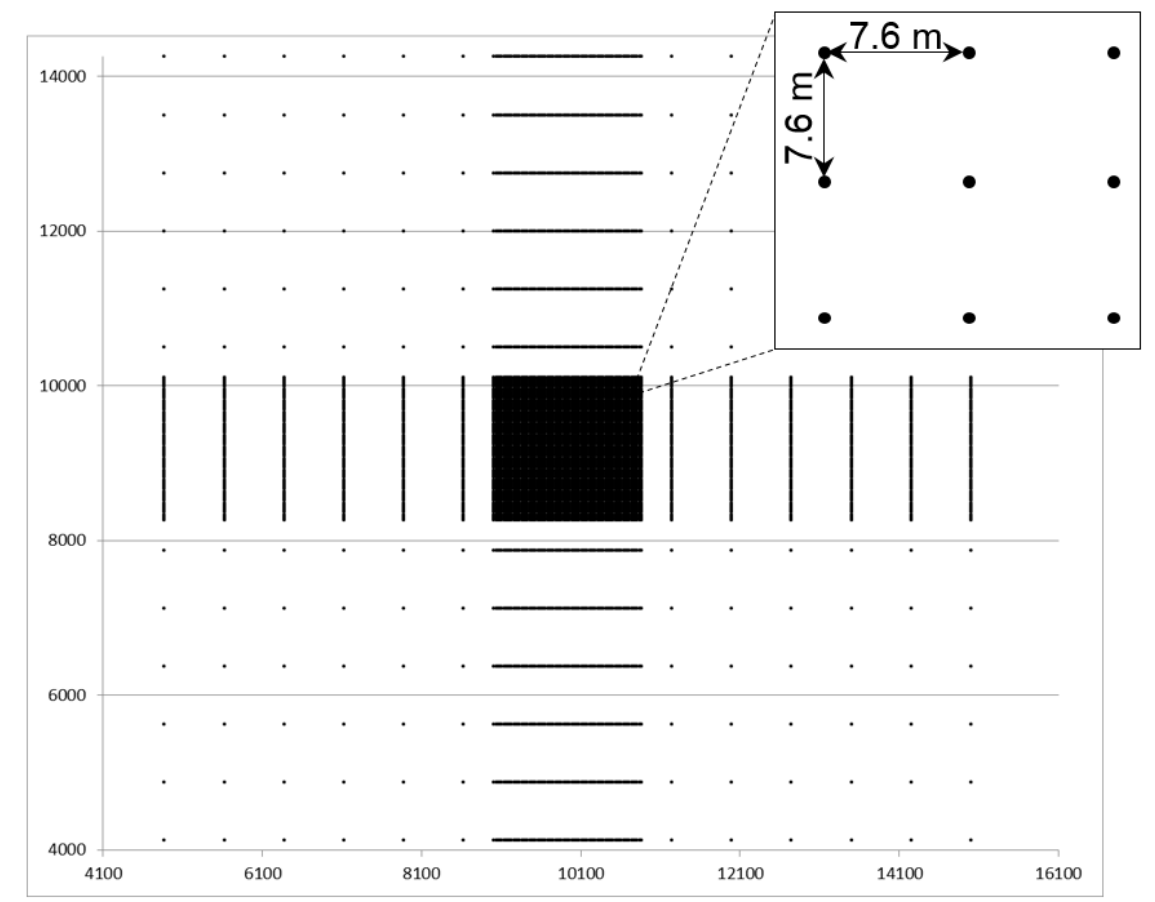

Figure 7-3: Plan view $34 \mathrm{~km}^{2}$ (13 mile $\mathrm{e}^{2}$ fluid simulation grid. Grid spacing is $229 \mathrm{~m}(750 \mathrm{ft})$, and refined to $7.6 \mathrm{~m}(25 \mathrm{ft})$ within a $564 \times 564 \mathrm{~m}(1850 \times 1850 \mathrm{ft})$ square around the injection and observation wells.

In the flow simulations performed by EERC two fluid components are considered, brine and $\mathrm{CO}_{2}$, and during injection, $\mathrm{CO}_{2}$ is permitted to dissolve into the brine.

Solubility of the $\mathrm{CO}_{2}$ in brine is computed using Henry's law in the manner put forth by Harvey (1996). Temperature and pressure are used to compute the density and viscosity of the resulting fluid mixtures using the methodology formulated in Rowe and Chou (1970) and Kestin et al. (1981). History matching of the injection data was performed 
using near real-time field measurements of injection rate, downhole pressure, and temperature, as well as spinner survey results from April 2015 (EERC, 2016). The spinner survey results suggested $\sim 90 \%$ of the $\mathrm{CO}_{2}$ entered the reservoir through the $2^{\text {nd }}$ and $4^{\text {th }}$ perforation zones, i.e., the top perforation zone in the upper Deadwood, and the single perforation zone in the lower Deadwood. The remaining $\sim 10 \%$ entered through the $1^{\text {st }}$ perforation zone, i.e., in the Black Island Formation. However, the spinner survey showed no $\mathrm{CO}_{2}$ entry through the $3^{\text {rd }}$ perforation zone. The spinner survey data were used to further refine permeability values close to the injection well to better match the pressure response and injection profile (EERC, 2016).

The reservoir flow simulations involved 120 layers and 12 lithologic units. The injection quantities included were $36 \mathrm{kt}, 60 \mathrm{kt}$, and $97 \mathrm{kt}$. The $36 \mathrm{kt}$ case corresponds to the quantity of $\mathrm{CO}_{2}$ present in the reservoir during the first monitor survey in February 2016, and the $97 \mathrm{kt}$ case was the closest available quantity to the $105 \mathrm{kt}$ in the second monitor survey in November 2016.

\subsection{2. $\quad$ Elastic properties and seismic modeling}

Fluid flow simulation parameters for each point in the reservoir include both static properties - fractional porosity, lithology, and model layer number - and dynamic properties - pressures and fractional $\mathrm{CO}_{2}$ saturations - for the 3 injection quantities. Lithology codes were assigned to every grid point in the baseline reservoir seismic model, with each code associated with a suite of log-based petrophysical properties median values of Vp, Vs, density and porosity (total and effective) - for each of the individual layers of the model. In the baseline 3D velocity model, the properties of each layer are kept constant. 
The properties determined from the pre-injection well logs are assumed to correspond to the baseline case where the reservoir is brine-saturated, and Gassmann fluid substitution calculations were undertaken to compute the $\mathrm{CO}_{2}$ saturated seismic models. The implementation of the Gassmann equations (Gassmann, 1951) was based on the formulations in Smith et al. (2003) and Zhu and McMechan (1990). For computing fluid mixture densities and bulk moduli, constant $\mathrm{P}$ and $\mathrm{T}$ values $\left(110^{\circ} \mathrm{C}\right.$ and $\left.35 \mathrm{MPa}\right)$ were assumed, and brine and $\mathrm{CO}_{2}$ properties were determined using the Batzle and Wang (1992) formulations. Mixed fluid densities were determined using Wood's equation (Wood, 1964). In the case of shale layers, velocity and density values were kept fixed throughout injection as the permeability of these layers is very low and very little $\mathrm{CO}_{2}$ is expected to enter these units. Additionally, Gassmann-type fluid substitution calculations are understood to be problematic for shale formations (Smith et al., 2003). In brief, the procedure used in this study involves first calculating the bulk modulus of the in-situ rocks using log-based properties. Then the bulk modulus of the drained porous rock frame and incoming fluid mixture are determined. These values are then used to compute a new saturated bulk modulus, and in-turn, P- and S-wave velocities.

One of the requirements for the injection seismic models is that points in the reservoir with $0 \% \mathrm{CO}_{2}$ saturation have velocities and densities that are equal to their corresponding points in the baseline model. Upon initially executing the Gassmann algorithm it was apparent that this requirement was not always met, likely in part due to measurement errors on the log-based parameters. To rectify this discrepancy, the bulk density of the saturated rock was computed using a mineral grain density that was allowed to vary until it yielded a value that agreed with the bulk density of the baseline 
rock in areas of $0 \% \mathrm{CO}_{2}$ saturation. Most units required a mineral grain density deviation of $\sim 1 \%$ from $\log$ values in order to match baseline densities, though the Deadwood B sandstone required a $12 \%$ deviation. A number of different parameters could have been allowed to vary, but mineral grain density was chosen due to the high degree of uncertainty associated with calculating this parameter from log measurements in the first place.

Fluid substitution calculations were performed at each grid point in the reservoir, regardless of the $\mathrm{CO}_{2}$ saturation associated with that point, then resampled in the $\mathrm{x}-\mathrm{y}$ (horizontal east-north) plane to uniform $4.86 \times 4.86 \mathrm{~m}$ bins throughout the entire reservoir volume. A uniform sample interval of $2 \mathrm{~m}$ was chosen in the z-direction (depth) to allow the DAS array to be represented in the seismic models. Three reservoir model files were generated for each injection quantity: P-wave velocity, S-wave velocity, and bulk density. Finally, the static 1-D overlying stratigraphy models were grafted on top of each reservoir model.

DAS employs optical fibers as a near-continuous sensor array by relating the backscattering of light pulses to strain imparted on the fiber via a wavefield. A detailed description of the mechanisms involved in DAS systems can be found in Mestayer et al., 2011, Miller et al., 2012, and Hornman, 2016. Recent advances in the technology have improved directionality by employing helically wound fibers (Kuvshinov, 2016). At the time of the permanent installation at Aquistore, however, the DAS system was limited to longitudinal sensitivity along a straight fiber. To replicate this limitation in the forward seismic modeling, only vertical particle velocity was analyzed. 
Seismic forward modeling was done using a finite-difference method for 3D elastic wave propagation. The SOFI3D (Bohlen, 2002) modeling program was used in this study, which uses an algorithm based on that described by Virieux (1986) and Levander (1988), and was implemented in a parallel MPI environment. Input parameters for this software are listed in Table 7-1. The program requires boundary conditions to converge on a unique solution. A 30-grid-point-thick "Perfectly Matched Layer" was placed around the boundary of the model to effectively dampen the waves near the edges of the volume (Komatitsch and Martin, 2007).

Simulations were run 3 times for each shot, corresponding to the 0,36 , and $97 \mathrm{kt}$ $\mathrm{CO}_{2}$ quantities. The baseline velocity and density model is shown in Figure 7-4. The $36 \mathrm{kt}$ and $97 \mathrm{kt}$ simulations included the 3D reservoir models produced during fluid substitution calculations.

Table 7-1: Finite Difference Model Parameters

\begin{tabular}{ll}
\hline Software & SOFI3D \\
Finite-difference Coefficients & $\begin{array}{l}\text { Holberg, } 8^{\text {th }} \text { order spatial operator, } 2^{\text {nd }} \text { order } \\
\text { temporal operator }\end{array}$ \\
$\begin{array}{l}\text { Dimensions in Voxels (East, } \\
\text { North, Depth) } \\
\text { Dimensions in Meters (East, } \\
\text { North, Depth) }\end{array}$ & $636,637,1748$ \\
$\begin{array}{l}\text { Voxel Resolution (East, North, } \\
\text { Depth) }\end{array}$ & $3091,3096,3496$ \\
Number of Receivers & $4.86 \mathrm{~m}, 4.86 \mathrm{~m}, 2 \mathrm{~m}$ \\
Recorded Particle Motions & 1383 spaced $2 \mathrm{~m}$ vertically \\
Wavefield Propagation Time & Vertical particle velocity \\
Timesteps, Sample Period & $3 \mathrm{~s}$ \\
Source Signal & $10000,0.0003 \mathrm{~s}$ \\
Absorbing Boundary & $\begin{array}{l}\text { Ricker wavelet, point source, } 38 \mathrm{~Hz} \text { center } \\
\text { frequency } \\
\text { Perfectly-matched layers, } 30 \mathrm{voxels}, 8 \% \\
\text { decay per voxel }\end{array}$ \\
\hline \hline
\end{tabular}




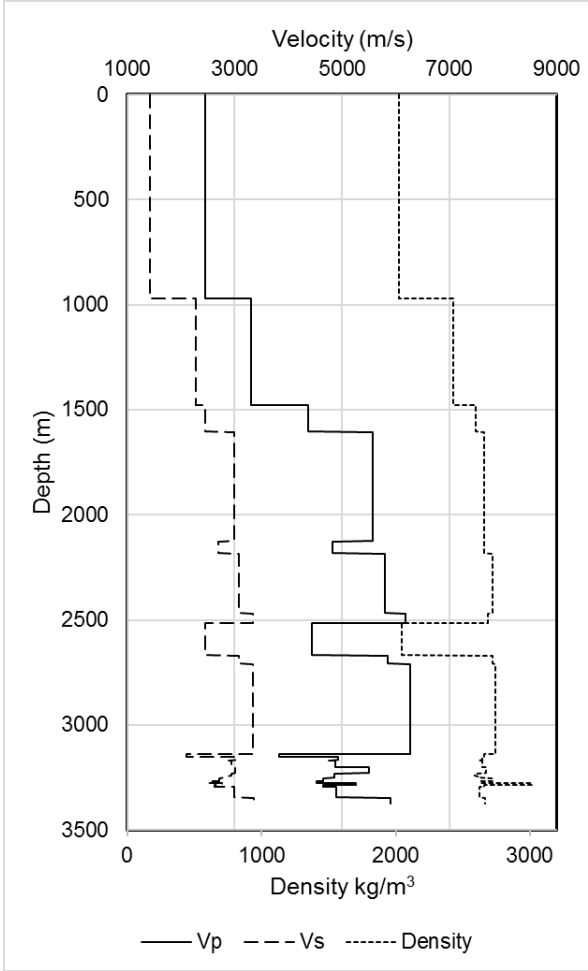

Figure 7-4: Baseline 1-D velocity and density model used as input for forward seismic modeling for the $0 \mathrm{kt}$ injection scenario.

\subsubsection{Acquisition of DAS field data}

For a detailed description of the DAS VSP survey procedures, refer to Harris et al. (2017). Acquisition parameters and an overview of the survey geometry can be found in Table 7-2.

The shot grid consisted of 670 shot locations over a $3 \times 3 \mathrm{~km}$ area around the injection well, with inline and crossline spacings of $72 \mathrm{~m}$ and $144 \mathrm{~m}$, respectively. Each source consisted of $1 \mathrm{~kg}$ of dynamite buried at a depth of $15 \mathrm{~m}$ to bypass the attenuating and unrepeatable effects of the overburden. Shot locations were known to have drifted from one survey to another as a result of infrastructure installed at the site throughout the monitoring schedule. Some shot locations shifted as far as $200 \mathrm{~m}$, and were inappropriate 
for 4D analysis. Most of these shots were located near the wells, and thus many nearoffset shot gathers were lost. The nearest useful shot was located $150 \mathrm{~m}$ away from the observation well. Additionally, it was difficult to precisely determine each shift due to measurement errors introduced during the baseline surveying. An unweighted average was used to produce the baseline data, while an optimally weighted average was used for the monitor data (Miller et al., 2016). This approach resulted in a signal-to-noise ratio improvement of $<10 \mathrm{~dB}$.

Table 7-2: Acquisition parameters for baseline and monitor DAS surveys

\begin{tabular}{cc}
\hline Parameter & \\
\hline \hline Sources & Shots \\
Grid size & 1 kg dynamite \\
Shot spacing & 670 shots over $3 \times 3 \mathrm{~km}$ \\
Shot depth & $72 \times 144 \mathrm{~m}$ (inline x crossline) \\
& $15 \mathrm{~m}$ \\
Interrogator & Sensors \\
Fiber & iDAS \\
Array length & Single mode VHS 3000 \\
Spacing & $2766 \mathrm{~m}$ \\
Gauge length & $2.036 \mathrm{~m}$ \\
Sampling frequency & $10 \mathrm{~m}$ \\
Trace length & $1000 \mathrm{~Hz}$ \\
Native response & $4 \mathrm{~s}$ \\
\hline \hline
\end{tabular}

\subsubsection{Processing of seismic data}

A summary of the processing flow and parameters can be found in Table 7-3, and an in-depth description of the field data processing flow can be found in Harris et al. (2017). The objectives of the devised processing flow for the synthetic data were to maintain a high level of repeatability between the baseline and monitor surveys, and to 
replicate the results of the field processing as well as possible. To this end, a parallel processing approach (Lumley et al., 2003; Li et al., 2011) was undertaken, wherein a single flow was duplicated for each vintage and run independently of the others.

Table 7-3: Processing steps and parameters. $\left({ }^{*}\right)$ indicates a process that is data dependent, $\left({ }^{\dagger}\right)$ indicates a process applied to synthetic data.

\begin{tabular}{|c|c|}
\hline Processing Step & Parameters \\
\hline $\begin{array}{c}\text { Prestack } \\
\text { Trimming and alignment of data sets } \\
\text { Polarity reversal of monitor data }\end{array}$ & \\
\hline $\begin{array}{l}\text { Omission of unrepeatable/shifted shots }{ }^{\dagger} \\
\qquad t^{2} \text { amplitude scaling }\end{array}$ & Shifts $>4.5 \mathrm{~m}$ omitted \\
\hline $\begin{array}{l}\text { Shot-based amplitude scaling* } \\
\text { Median filtering of direct wave }{ }^{* \dagger} \\
f-k \text { filtering of converted waves }{ }^{\dagger}\end{array}$ & $\begin{array}{l}\text { Based on RMS of first arrivals }(100-1000 \mathrm{~m}) \\
\qquad 51 \text { trace filter }\end{array}$ \\
\hline Shot-based deterministic deconvolution ${ }^{* \dagger}$ & $200 \mathrm{~ms}$ filter, $1 \%$ prewhitening, $10-15-80-90 \mathrm{~Hz}$ \\
\hline \multicolumn{2}{|l|}{ Shot-based residual statics* } \\
\hline 3D Kirchhoff depth migration ${ }^{\dagger}$ & $\begin{array}{c}12.5 \times 12.5 \times 2 \mathrm{~m} \text { bins, } \pm 15^{\circ} \text { aperture, identical } \\
\text { velocity model for each vintage }\end{array}$ \\
\hline \multicolumn{2}{|l|}{ Poststack } \\
\hline Phase-time matching* & $1500-3100 \mathrm{~m}$ design window \\
\hline Shaping filtering* & $1500-3100 \mathrm{~m}$ design window \\
\hline Amplitude normalization* & $1500-3100 \mathrm{~m}$ design window \\
\hline
\end{tabular}

Although the synthetic data was generated without additive noise, the processing flow was required to include the same limitations due to non-repeatable noise that affected the field data. The most notable examples of this requirement include: shot omissions, channel omissions, application of narrow band-pass filters, and using targeted windows to design various operators. As seen in Table 7-3, several of the processing steps applied to the field data were omitted from the synthetic data workflow. These were steps known to be unnecessary to maintain repeatability or replicate field data limitations. 
As there was no shot variability or overburden heterogeneities in the model, it was not necessary to correct for statics or to cross-equalize amplitudes. Nor was it necessary to align channel depths or trim surface segments of the fiber. Examples of a near-offset baseline shot gather and monitor-baseline difference gathers at different stages throughout the processing flow are shown in Figure 7-5 and Figure 7-6.

The modeled plume is entirely located in a specular blind-spot resulting from the limited coverage of the VSP geometry. It was determined in Harris et al. (2017) that a relatively wide migration aperture of $\pm 15^{\circ}$ is necessary to image the reservoir in the vicinity of the injection well. The study also found that the best results were attained using only data from the lowermost $1800 \mathrm{~m}$ of the fiber, thus this study uses the same subset for imaging.

An assessment of anomalies presumably due to the presence of $\mathrm{CO}_{2}$ will be made using root-mean-square (RMS) values and normalised RMS (nRMS) values in the migrated difference volumes:

$$
n R M S=\frac{2 \times R M S_{M-B}}{R M S_{B}+R M S_{M}}
$$

where subscripts $B$ and $M$ represent baseline and monitor data, respectively. The chief advantage of using nRMS is that it expresses a change in the signal strength as a fraction of the original signal strength. This is appropriate for identifying areas of $\mathrm{CO}_{2}$ saturation, as the induced change in acoustic impedance is itself proportional to the baseline acoustic impedance. One consideration when using nRMS to characterize plume geometry is that low seismic amplitude areas that contain processing or imaging artefacts can exhibit a greatly exaggerated response. Therefore anomalies should be interpreted with both RMS 
and nRMS volumes. The nRMS ranges from 0 to 2 , with 0 corresponding to identical datasets, and 2 corresponding to polarity reversed datasets. Often, nRMS is used to quantify repeatability in 4D studies. Using modern surface seismic acquisition and processing techniques, nRMS values below 0.6 are considered good while values below 0.2 are considered excellent (Lumley, 2009). In the following section, analysis of the $\mathrm{CO}_{2}$ based anomaly will be conducted with consideration of the repeatability limitations ascertained in the field data study. 


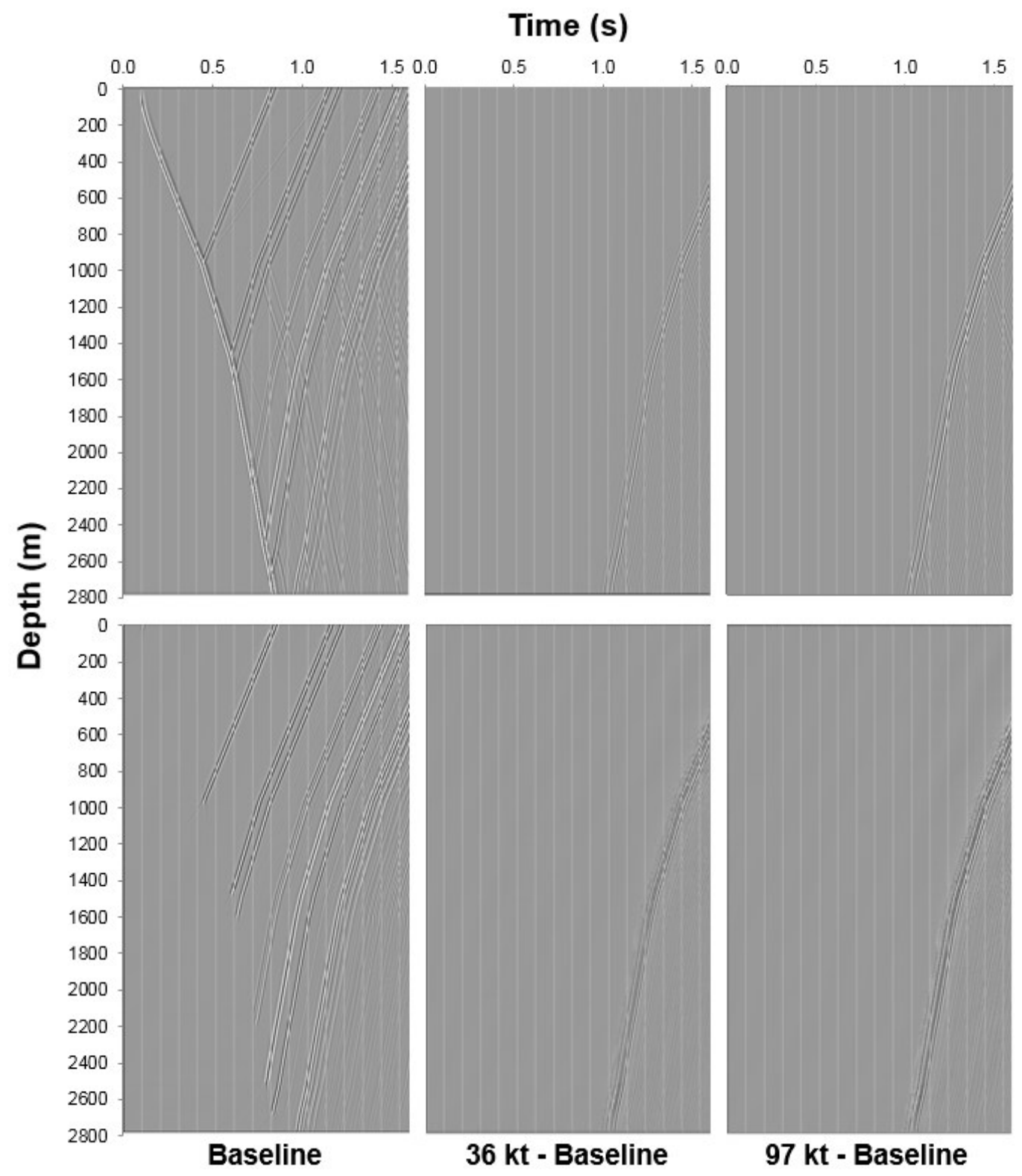

Figure 7-5: Example of a near-offset synthetic shot gather progressing through the processing sequence. The top row shows the raw baseline gather and its time-lapse differences between the monitor gathers. The bottom row shows the gathers after the removal of downgoing and converted wave modes via median and $f-k$ filters. Differences have been amplified by a factor of 10 relative to the baseline gather. 


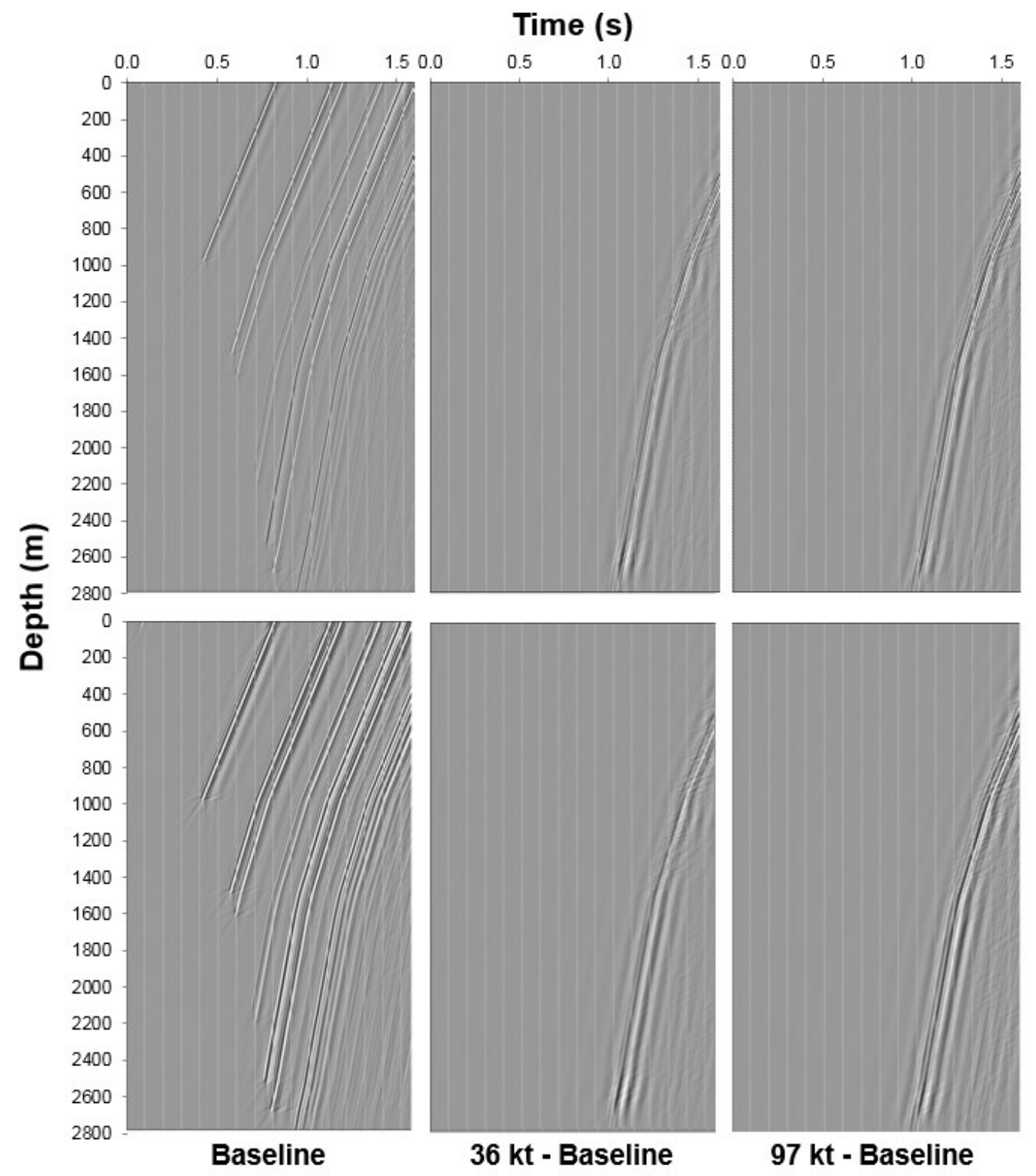

Figure 7-6: Continued example of a near-offset synthetic shot gather progressing through the processing sequence. The top row shows the baseline gather and its time-lapse differences between the monitor gathers after deterministic deconvolution has been applied. The bottom row shows the gathers after the application of a bandpass filter to reduce the bandwidth to that of the field data. Differences have been scaled $x 10$ relative to baseline gather. 


\subsection{Results}

Figure 7-7 shows cross-sections and depth slices through the injection well from the $3 \mathrm{D}$ simulation saturation results at two stages of injection. After $36 \mathrm{kt}$, the plume is present in the $1^{\text {st }}, 2^{\text {nd }}$, and $4^{\text {th }}$ perforated zones, and extends laterally $\sim 150 \mathrm{~m}$ from the injection well. The $2^{\text {nd }}$ and $4^{\text {th }}$ zones (upper and lower Deadwood) have the highest $\mathrm{CO}_{2}$ saturations, with saturations of $0.50-0.70$ in the vicinity of the injection well. The $1^{\text {st }}$ zone (Black Island) has lower saturations ranging from $0.30-0.50$ and hosts a narrower plume extending $<100 \mathrm{~m}$ from the injection well. After $97 \mathrm{kt}$ of injection, the plume has expanded laterally with no vertical migration through the shale units. The plume extends $\sim 250 \mathrm{~m}$ from the injection well at $0.50-0.70 \% \mathrm{CO}_{2}$ saturations in the $4^{\text {th }}$ zone, and only $\sim 150 \mathrm{~m}$ at $0.30-0.50$ saturation in the $1^{\text {st }}$ zone. 


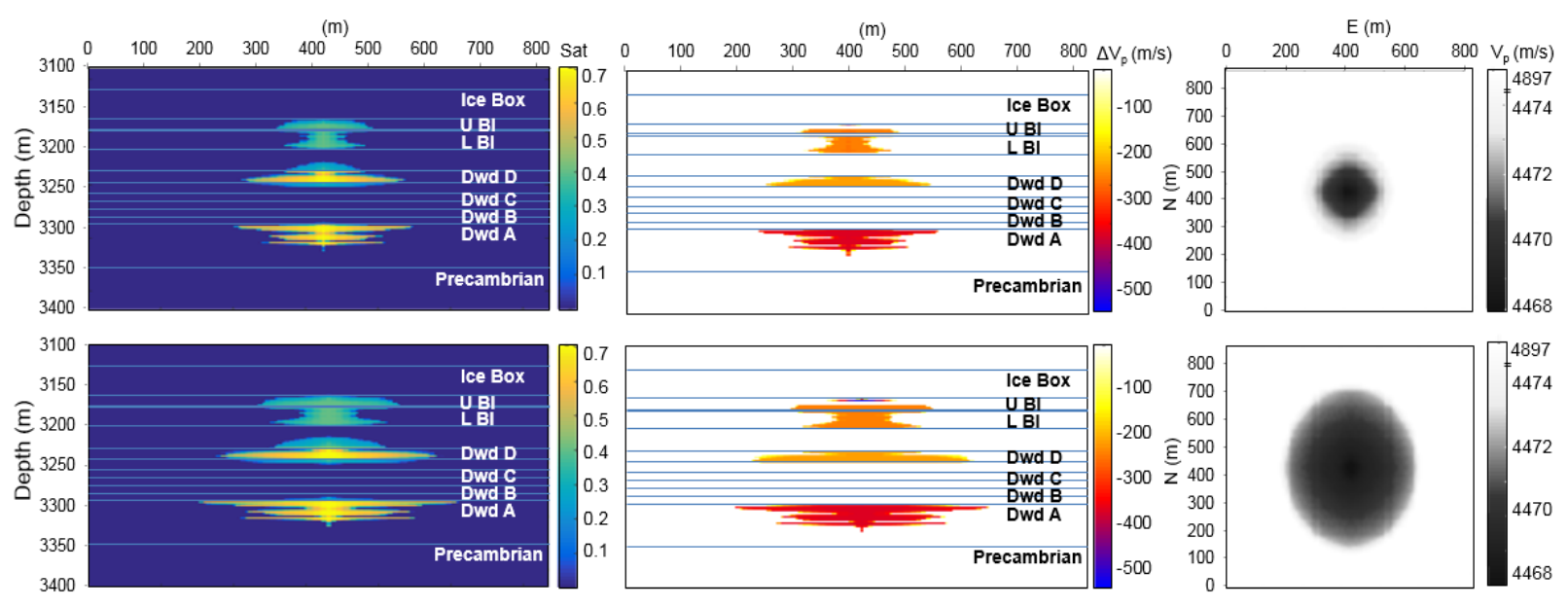

Figure 7-7: South-north cross-sections of the reservoir through the injection well showing saturation (left) and P-wave velocity difference from baseline (center), and plan view of Pwave velocity at $3300 \mathrm{~m}$ (right). Values for $36 \mathrm{kt}$ and $97 \mathrm{kt}$ of $\mathrm{CO}_{2}$ are shown in the top row and bottom row, respectively. Deadwood C and D comprise the upper Deadwood, and Deadwood A and B comprise the lower Deadwood.

Figure 7-7 also shows cross-sections of $\mathrm{P}$-wave velocity difference relative to preinjection velocities (center) and P-wave velocity depth slices for the 36 and $97 \mathrm{kt}$ scenarios (right), as determined via Gassmann's methodology. The $1^{\text {st }}$ and $2^{\text {nd }}$ perforated zones show velocity decreases of $200-250 \mathrm{~m} / \mathrm{s}$ for both scenarios, whereas velocity decreases of up to $400 \mathrm{~m} / \mathrm{s}$ occur in the $4^{\text {th }}$ zone. The depth slice at $3300 \mathrm{~m}$ was taken through the most expansive portion of the plume. The P-wave velocity does not vary significantly within the plume, increasing only $\sim 8 \mathrm{~m} / \mathrm{s}$ from the injection well to its outer edges. However, the velocity increases abruptly outside the limits of the plume (note the discontinuity in the greyscale color bar). This is a result of the low sensitivity of Gassmann-derived P-wave velocity changes when $\mathrm{CO}_{2}$ saturations are moderate or high, as well as of the abrupt change in $\mathrm{CO}_{2}$ saturations at the periphery of the plume as 
predicted by the flow simulations. Plume migration is approximately symmetrical, with elongation along the north-south axis.

Gassmann's methodology relies on several assumptions about the reservoir, namely that the rock is homogeneous, elastic, and isotropic, and that the pores are interconnected (Wang, 2001). The calculations also assume that shear modulus does not vary with fluid saturation, and that the fluids mix homogenously in the pore space.

Several of the assumptions are violated for clay-rich rocks, and shear modulus is known to vary with increasing saturation as the shale strengthens and stiffens (Ghorbani et al., 2009). In this study, fluid substitution effects were set to zero for the shales even though the flow simulations identify low saturation values in some of the shale units.

Figure 7-8 shows the Gassmann-derived predicted behavior of seismic velocity, density, and P-wave impedance with increasing $\mathrm{CO}_{2}$ saturation in the three perforated zones that hosted $\mathrm{CO}_{2}$. Maximum $\mathrm{CO}_{2}$ saturations of 0.40 are observed in the $2^{\text {nd }}$ perforated zone whereas values in excess of 0.70 occur in zones 1 and 4 . In general, Pwave velocity and impedance decrease by values ranging between 5-9\% and 6-10\%, respectively, whereas density decreases are less than $1.2 \%$. In contrast, S-wave velocity increases slightly by less than $0.6 \%$. The large changes in P-wave velocity are expected due to the significant change in bulk modulus upon replacement of brine with $\mathrm{CO}_{2}$, whereas the comparable density of these fluids and low porosity of the rocks limits the associated rock density changes. The sharpest change in P-wave velocity is observed between 0-0.15 saturation, and levels-off considerably for saturations greater than 0.40. At 0.40 saturation, P-wave impedance has decreased by approximately $6 \%, 6 \%$, and $9 \%$ for perforated zones 1,2 , and 4 , respectively. The greater change observed for zone 4 is 
due in large part to the relatively low bulk modulus of its porous rock frame. By replacing the in-situ brine with a fluid mixture of lower compressional strength, the bulk modulus of the saturated rock is proportionally more largely affected than the rocks with a higher porous rock frame bulk modulus.

Due to the enhanced P-wave velocity sensitivity to $\mathrm{CO}_{2}$ saturation and because zone 4 exhibits some of the highest $\mathrm{CO}_{2}$ saturations in the reservoir in both the $36 \mathrm{kt}$ and $97 \mathrm{kt}$ scenarios, it is therefore expected that this zone will yield the largest magnitude response in the final seismic sections.

Examples of unprocessed near-offset shot gathers are shown in Figure 7-9 to compare synthetic and field data. A zero-phase wavelet was used to generate the synthetic data whereas the field data are minimum-phase. Aside from the phase difference, the synthetic data is generally in good agreement with the field data with regard to arrival times and moveout of the direct waves and strong reflectors. A guided wave between $1000-1450 \mathrm{~m}$ in the field gathers is not present in the synthetic gathers. This discrepancy is due to the presence of a poor quality cement zone in the borehole that was not replicated in the model. This event, in addition to tube waves and downgoing multiples in the shallow areas of the gather, does not likely contribute significantly to the final processed volume as these wave-modes are removed during median and $f-k$ filtering. The reflection from the top of the reservoir is easily identifiable in the noise-free synthetic gathers, as well as additional reflections from within the reservoir. These reflections are visible in the $36 \mathrm{kt}$ field data as well albeit with substantially more noise. In the baseline field gather, the top of the reservoir is faintly visible whereas deeper reflections are obscured due to low signal-to-noise ratio as compared to the $36 \mathrm{kt}$ field 
data. This difference is the result of improved noise cancellation techniques being applied during field acquisition in the latter case.
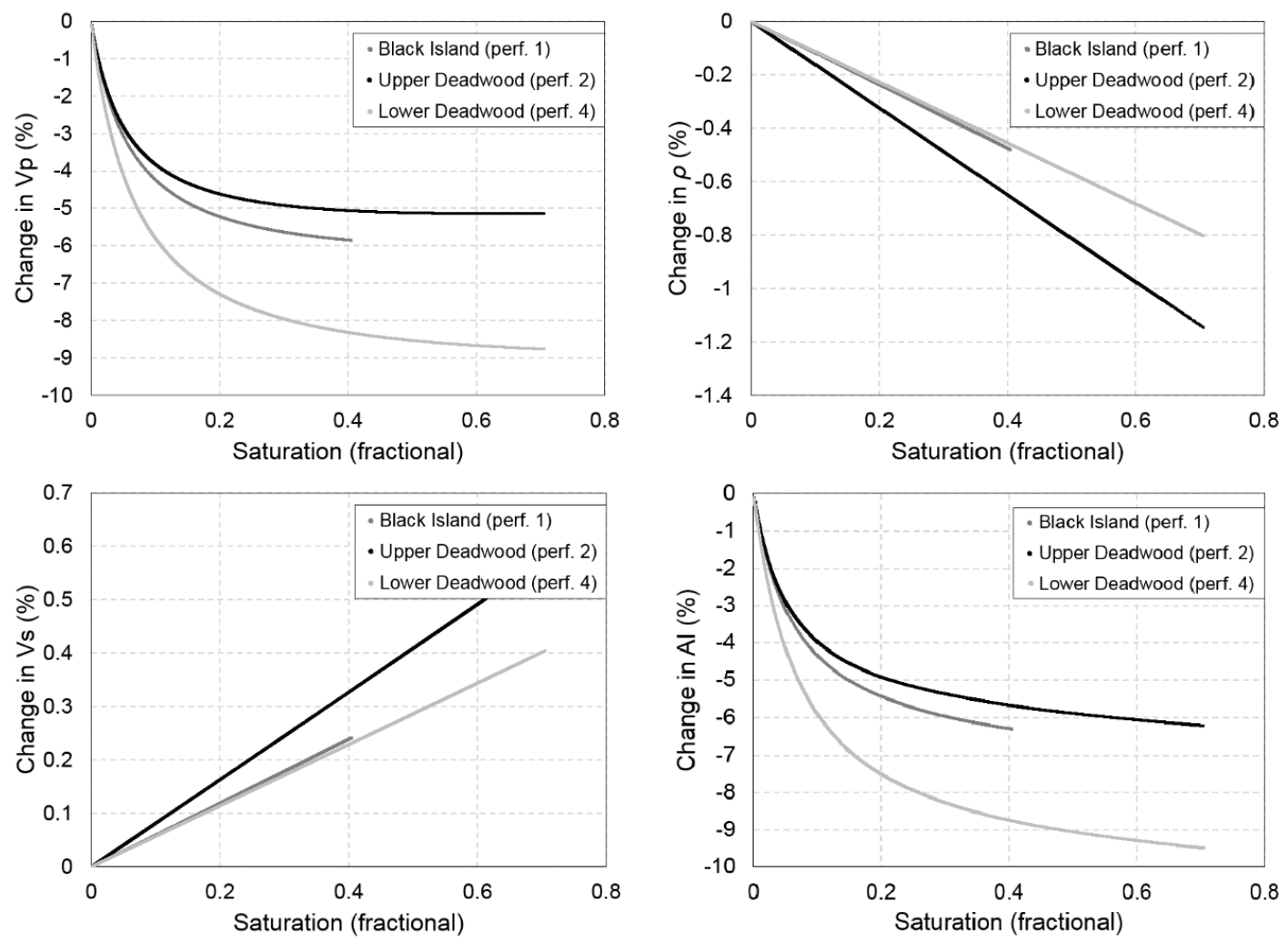

Figure 7-8: Changes in (clockwise from top-left) P-wave velocity, density, P-wave acoustic impedance (AI), and S-wave velocity as a function of $\mathrm{CO}_{2}$ saturation for each zone located adjacent to a well perforation zone. 


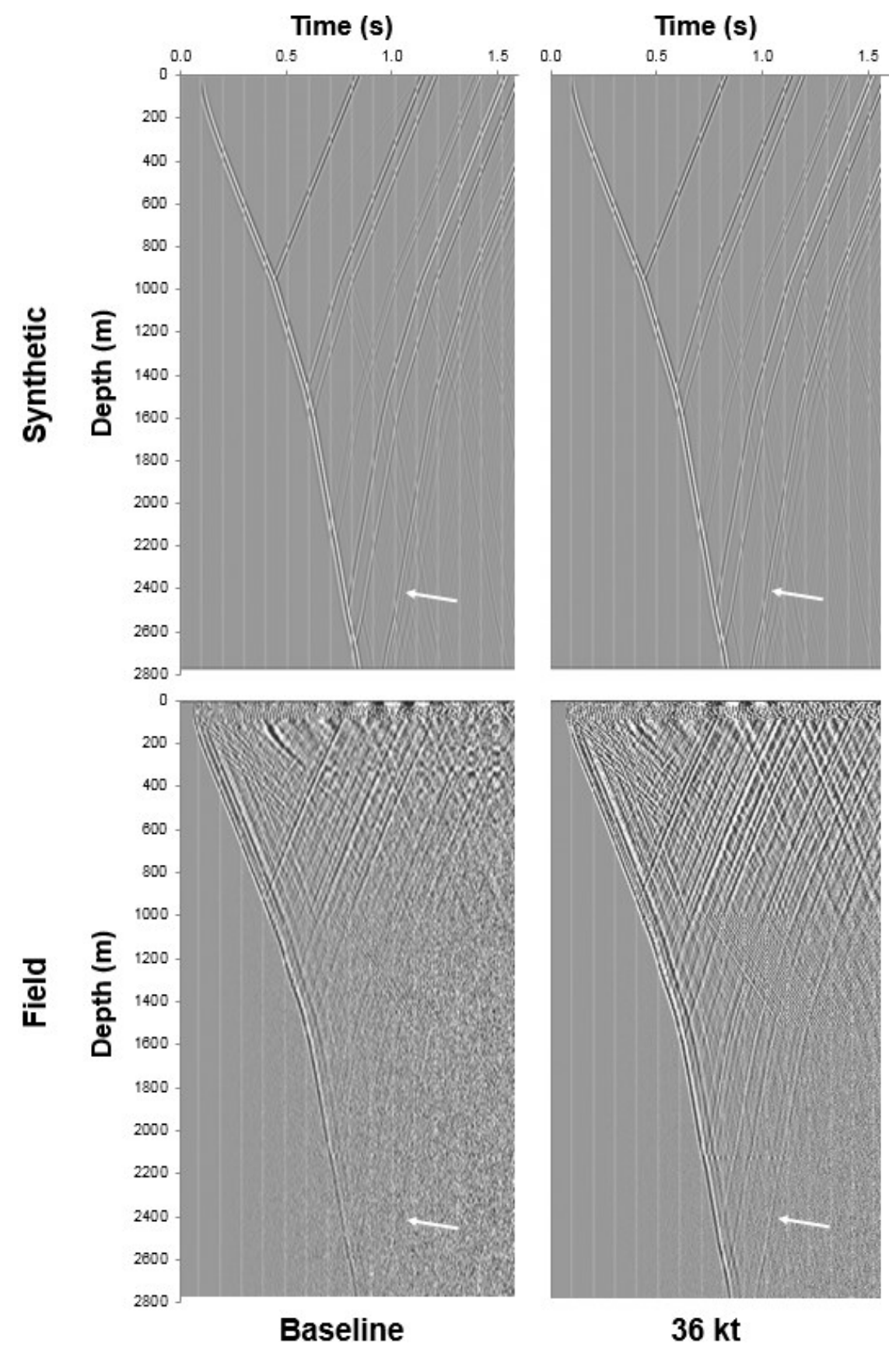

Figure 7-9: Comparison of near-offset shot gathers for the baseline and 36 kt injection scenarios. The FDTD synthetic data and field data are shown in the top and bottom rows, respectively. The reflection from the top of the reservoir is indicated on each gather by a white arrow. 
For the purposes of VSP imaging, a subset of the field data was selected that included only the most repeatable and high signal-to-noise ratio shots. After processing, these 50 shots were migrated and stacked into the final volume interpreted in Harris et al. (2017). A comparison of this field data stack with the equivalent synthetic stack is shown in Figure 7-10. A bulk depth and phase shift was applied to the volumes to align horizons at the top of the reservoir, and the synthetic data was scaled to equalize the Ice Box shale amplitude to that of the field data.

To demonstrate the ability of the workflow to image the $\mathrm{CO}_{2}$ distribution in a noise-free environment, Figure 7-11 shows the $\mathrm{CO}_{2}$ saturations predicted by the flow simulations alongside the nRMS of the time-lapse seismic differences. The strongest response in the cross-sections are in the lower Deadwood $(\sim 3300 \mathrm{~m})$ for both the $36 \mathrm{kt}$ and $97 \mathrm{kt}$ scenarios. The $36 \mathrm{kt}$ anomaly extends $100-150 \mathrm{~m}$ from the injection well, whereas the $97 \mathrm{kt}$ synthetic anomaly extends $\sim 250 \mathrm{~m}$. This is in approximate agreement with the saturation model (black outline). The magnitude of the nRMS anomalies associated with the upper Deadwood ( 3230 $\mathrm{m})$ and the Black Island $(\sim 3180 \mathrm{~m})$ are comparatively small. In each scenario, these anomalies do not exceed nRMS $\sim 0.15$, and their shape and size do not correlate well with the saturation distribution. 


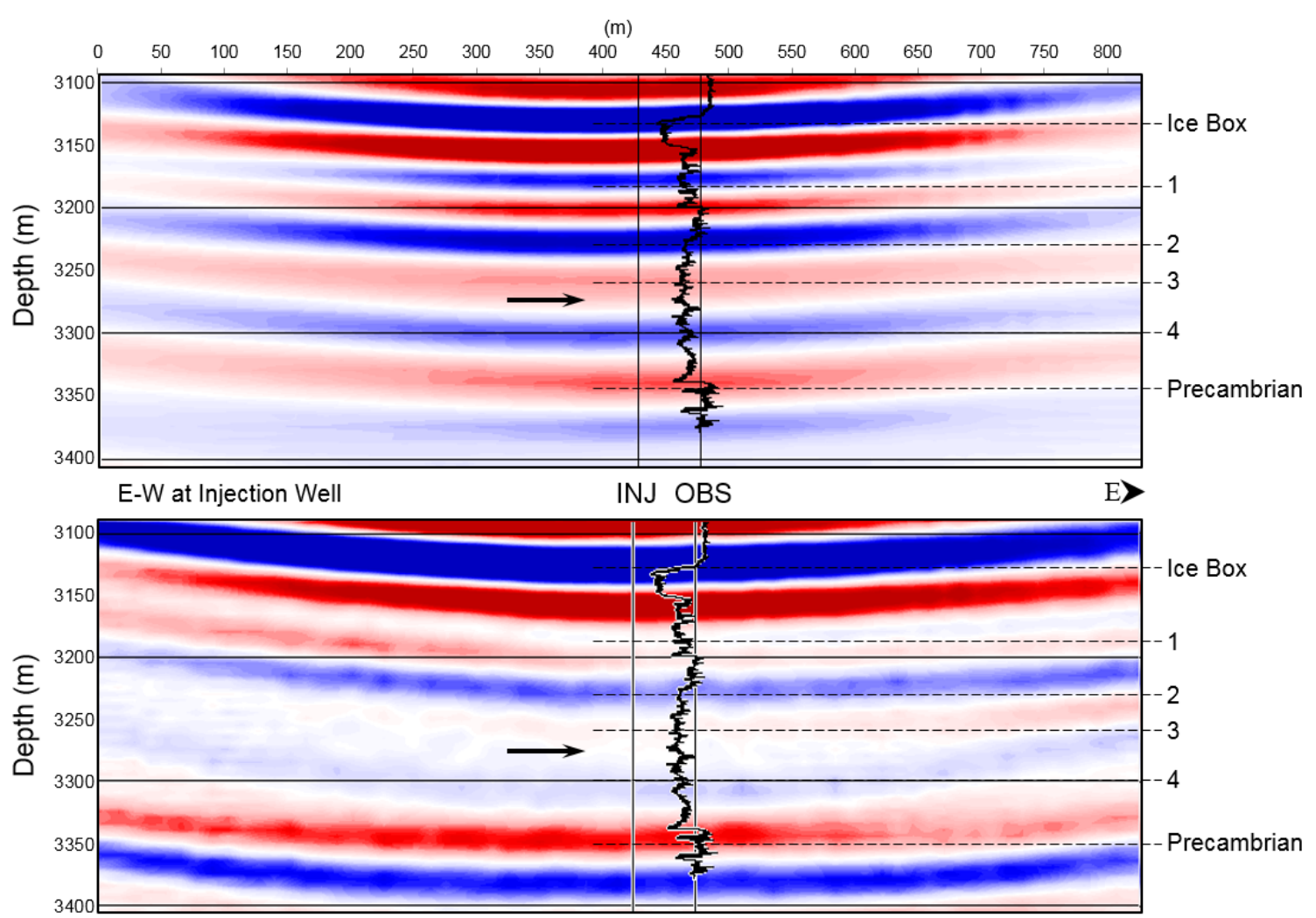

Figure 7-10: Comparison of migrated 50-shot stack of baseline FDTD synthetic data (top) and field data (bottom). The figure shows a close-up of the reservoir in a west-east crosssection through the injection well, with the observation well and P-wave sonic log projected into the section. Perforated zones are labelled 1 through 4, and correspond to the lower Black Island, upper Deadwood (I), upper Deadwood (II), and lower Deadwood, respectively. Black arrows indicate the location of a low-amplitude zone present in the field section, but not in the synthetic section. The concave upward shape of the horizons is a migration artifact related to the sparser shot coverage further from the borehole and to the use of a wide migration aperture. 


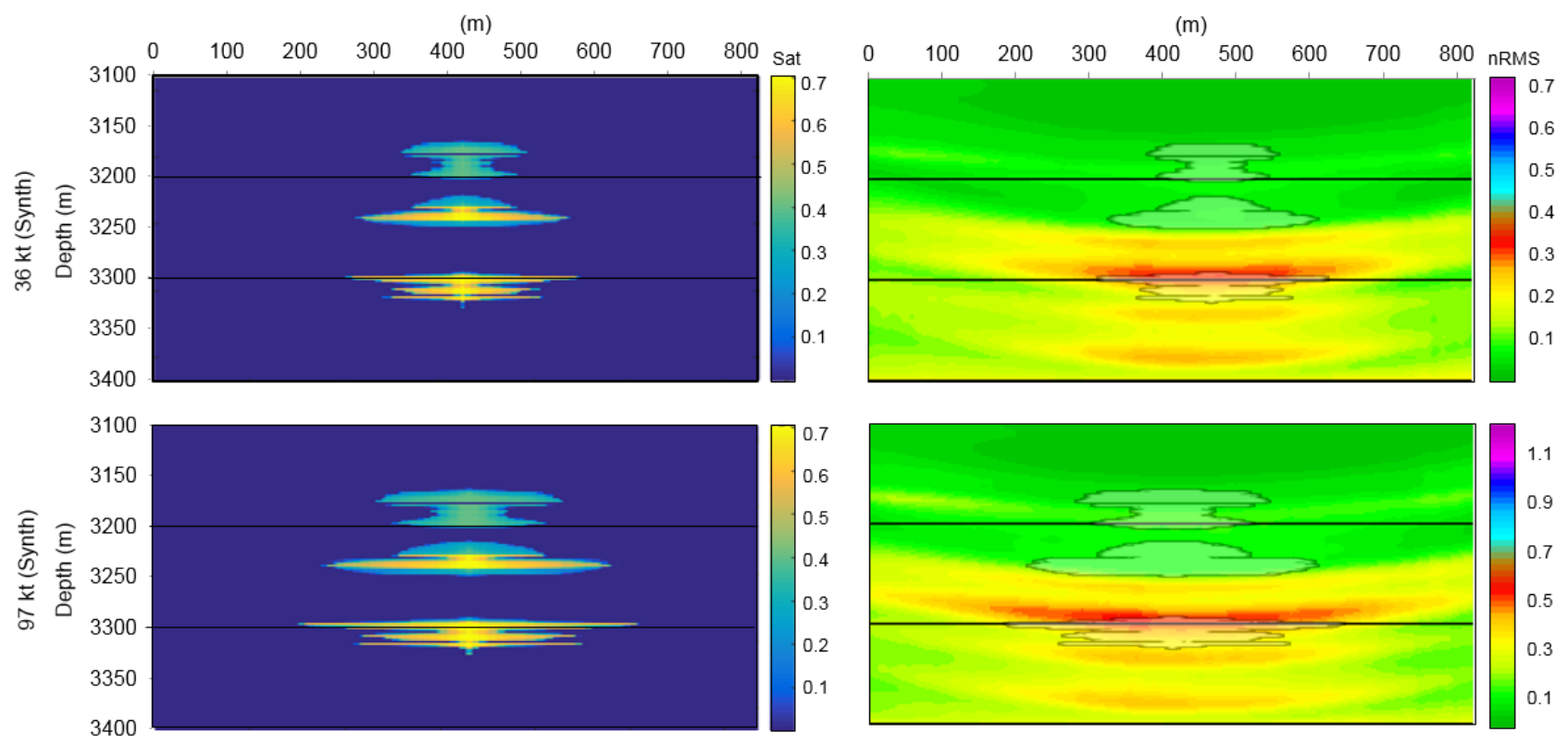

Figure 7-11: West-east cross-sections through the injection well for $36 \mathrm{kt}$ synthetic data (top), and $97 \mathrm{kt}$ synthetic data (bottom). Sections show $\mathrm{CO}_{2}$ saturations predicted from the flow simulations (left), and nRMS of the time-lapse seismic differences (right) in the reservoir. Note that the nRMS sections have been variably scaled to better highlight the plume for each scenario.

Cross-sections from both field and synthetic data difference volumes are shown in Figure 7-12. It is difficult to make any interpretations about the shape or size of the plume from the raw difference sections (top row of Figure 7-12). This is especially true for the field data since unrepeatable noise is present and obscures the boundaries of the plume signature. However, the strongest time-lapse difference in the field data resides at $\sim 3300 \mathrm{~m}$ (black arrow), corresponding to the $4^{\text {th }}$ perforated zone, which is in agreement with the synthetic data. Application of a $50 \mathrm{~m}$ RMS sliding window to the difference volumes allows for a better visualization of the anomalous amplitudes as seen in the middle row of Figure 7-12. The strongest RMS difference anomaly again occurs at $\sim 3300$ 
$\mathrm{m}$ (black arrow) in each scenario with a much weaker RMS difference anomaly occuring at $\sim 3200 \mathrm{~m}$ (white arrow) in the synthetic volumes. This upper anomaly corresponds to a location within $1 / 4$ wavelength of both the $1^{\text {st }}$ and $2^{\text {nd }}$ perforated zones. No significant RMS difference anomaly is observed in the field section at this depth. The magnitude of the field data anomaly is $<0.050$, which is somewhat stronger than the $<0.035$ in the synthetic volume for the same injected quantity, but similar to the $<0.050$ for the $97 \mathrm{kt}$ synthetic volume. 


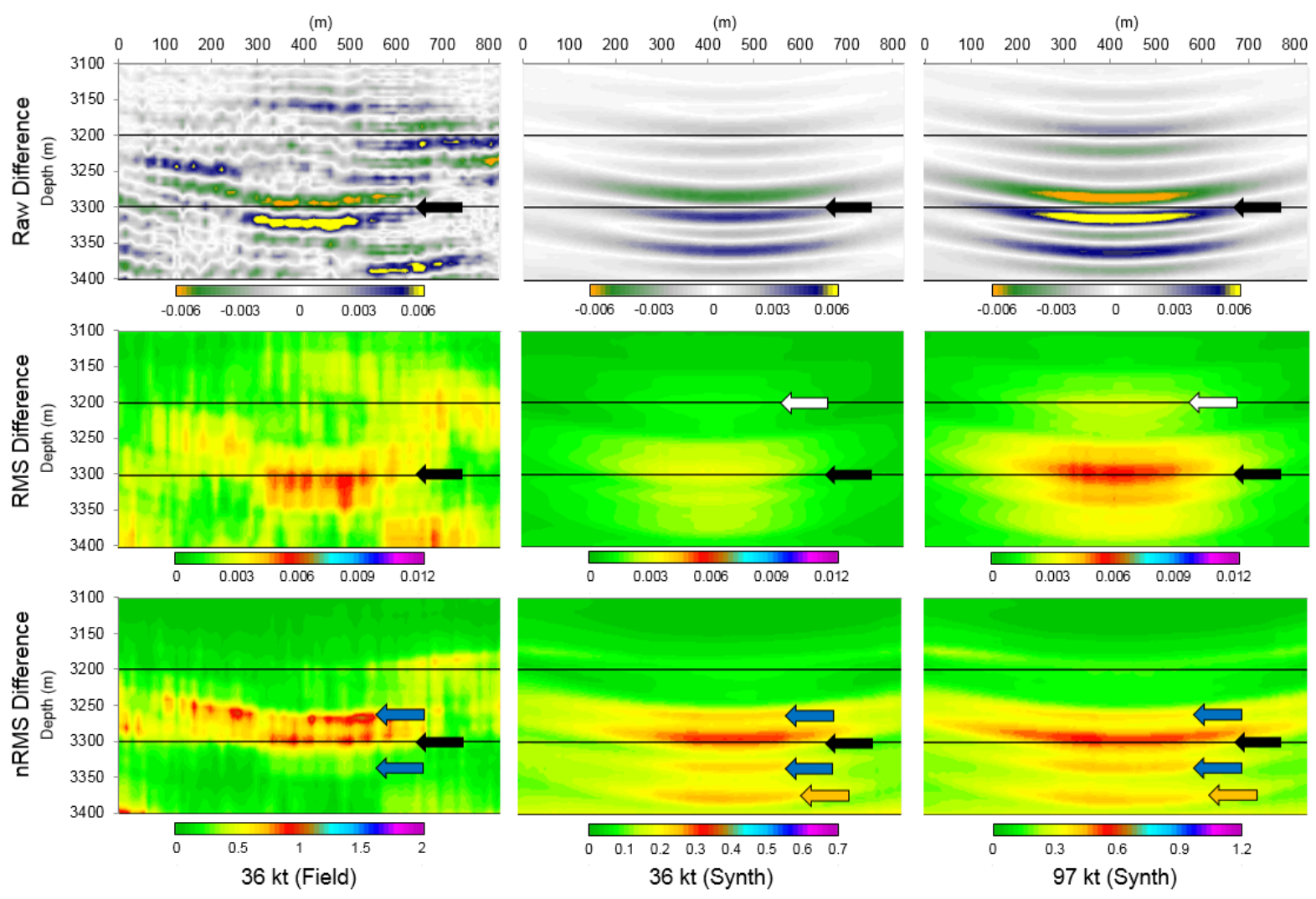

Figure 7-12: West-east cross-sections through the injection well for $36 \mathrm{kt}$ field data (left), 36 kt synthetic data (center), and $97 \mathrm{kt}$ synthetic data (right). Sections show raw time-lapse differences (top), RMS differences (center), and nRMS of the differences (bottom) in the reservoir. Note that the nRMS sections have been variably scaled to better highlight the plume for each scenario. Refer to body of the text for descriptions of arrow locations.

The bottom row of Figure 7-12 shows the nRMS-of the time-lapse differences. The magnitude of the lower Deadwood (black arrow) nRMS anomaly for the field data $(<0.90)$ is significantly larger than that of the $36 \mathrm{kt}$ scenario for the synthetic data $(<0.35)$, and even exceeds that of the $97 \mathrm{kt}$ scenario for the synthetic data $(<0.55)$. In each section, there is a significant signal at $\sim 3260 \mathrm{~m}$. Figure $7-7$ shows that there is no $\mathrm{CO}_{2}$ present here, nor a corresponding change in elastic properties. This can be explained as 
an artifact caused by the side-lobes (blue arrows) of the band-limited wavelet in the synthetic sections, but the signal is considerably stronger in the field section, and will warrant further discussion later. In the synthetic sections, there are also significant signals at $\sim 3330$ and $\sim 3375 \mathrm{~m}$. The signal at $\sim 3330 \mathrm{~m}$ is below any significant $\mathrm{CO}_{2}$ saturation, and may also be attributed to the side-lobes of the wavelet. The signal at $\sim 3375 \mathrm{~m}$ (orange arrow) is within the Precambrian basement, and is likely a result of a time delayed wavelet, as seen in Figure 7-13, that traveled through the overlying $\mathrm{CO}_{2}$. The amplitude difference brought upon by this small time shift (and subsequent $<10 \mathrm{~m}$ depth shift) was normalized by a relatively weak basement signal (3375 $\mathrm{m}$ in Figure $7-10$ ), and thus produced a nRMS anomaly.
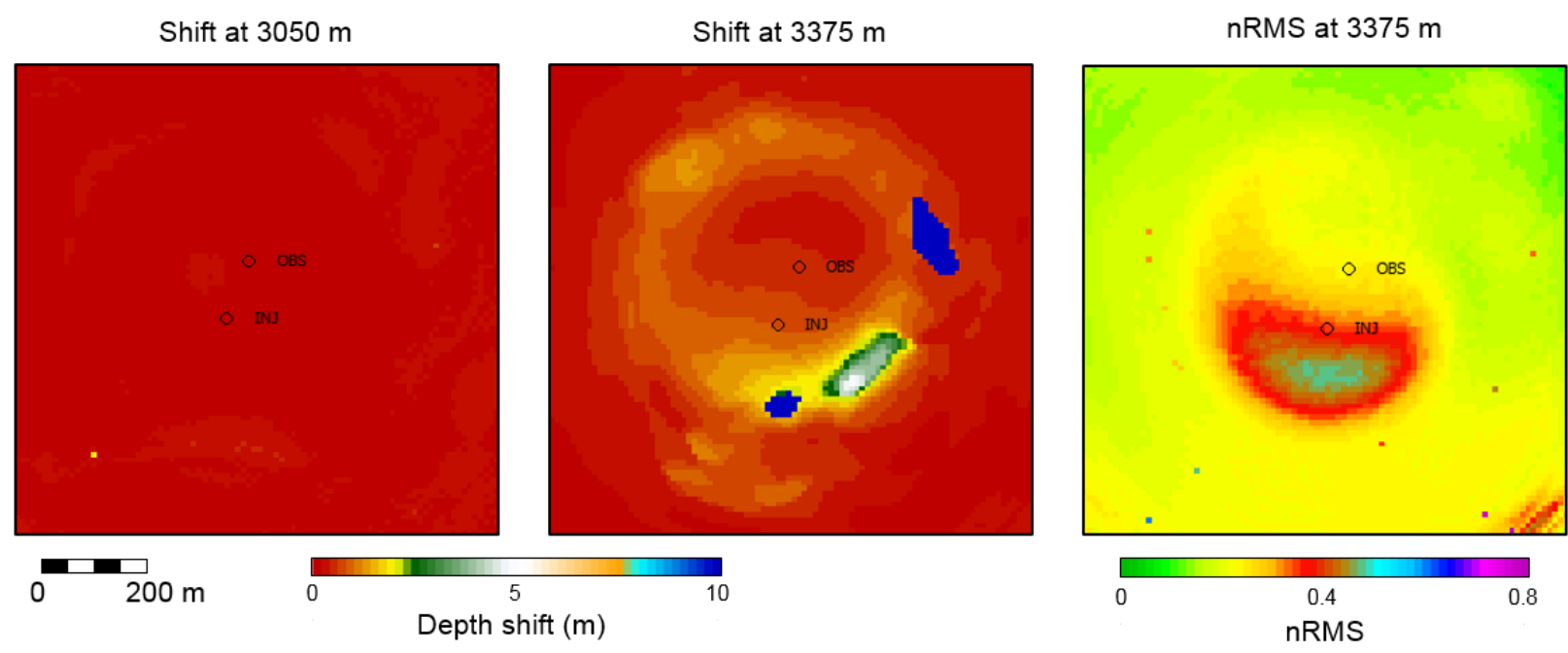

Figure 7-13: Depth shifts between baseline and $97 \mathrm{kt}$ synthetic data for horizons above reservoir (left) and below reservoir (center), and nRMS anomaly below reservoir (right).

Figure 7-14 presents depth sections taken at $3300 \mathrm{~m}$ that show RMS and nRMS values of the time-lapse differences and can be used to interpret the lateral extent of the plume. As consistent with the elastic models shown in Figure 7-7, the plume extends 
approximately radially from the injection well in the synthetic RMS and nRMS volumes.

However, in the field data volumes the plume trends northward, with very little signal visible south of the injection well.
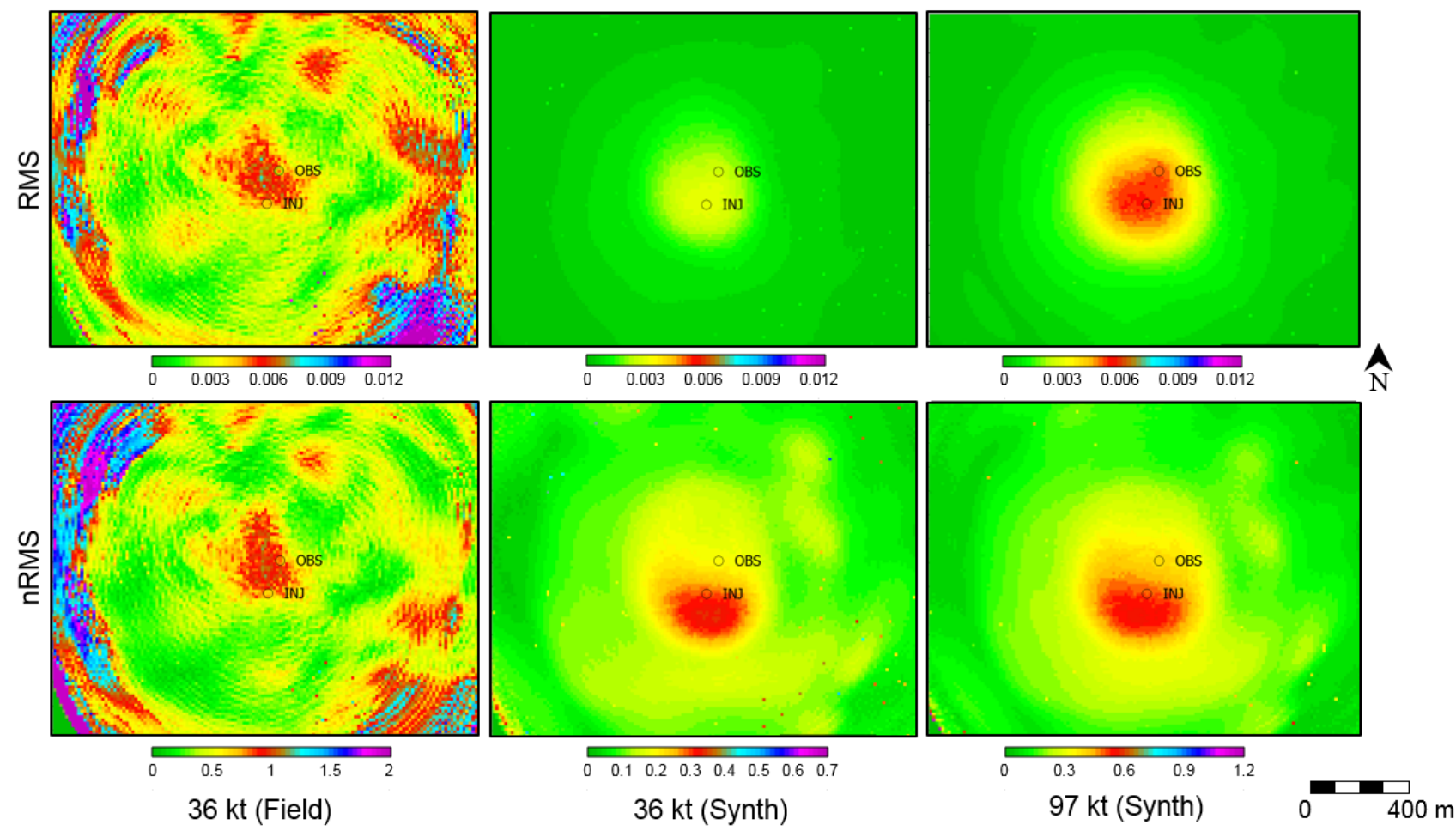

Figure 7-14: 10 m-thick depth sections through the RMS (top) and nRMS difference volumes (bottom) in the lower Deadwood (3300 m). Note that the nRMS sections have been variably scaled to better highlight the plume for each scenario.

\subsection{Discussion}

Based on the behavior of acoustic impedance with increasing saturation in Figure 7-8, and the uniformity of the time-lapse P-wave changes in Figure 7-7, it can be inferred that the seismic response is fairly insensitive to increases in $\mathrm{CO}_{2}$ saturations greater than 0.25. For example, the modeled P-wave impedance in the lower Deadwood only decreases $\sim 1 \%$ when the $\mathrm{CO}_{2}$ saturation increases from 0.25 to 0.60 . Additionally, the 
modeled saturations themselves fall off rapidly, in many locations decreasing from 0.50 to 0 over $\sim 10 \mathrm{~m}$. It is therefore expected that, in the seismic difference volumes, the signatures of $\mathrm{CO}_{2}$ plumes should be similarly of uniform magnitude and exhibit welldefined boundaries. When comparing the plume saturations with their respective simulated seismic nRMS responses in Figure 7-11, it can be seen that the responses do not vary significantly over the plume location, that is, the nRMS values are $\sim 0.35$ over $\sim 300 \mathrm{~m}$ for the $36 \mathrm{kt}$ scenario, and the nRMS values are $\sim 0.55$ over $\sim 500 \mathrm{~m}$ for the $97 \mathrm{kt}$ scenario. The seismic responses, however, gradually taper off laterally over the next several hundred meters, somewhat obscuring the plume boundaries. This gradual tapering off is a result of the inability of the Kirchhoff migration to collapse all diffracted energy to its origin. In future projects, this can be improved with a denser shot grid, or more sophisticated migration techniques (i.e. one that can handle complex structures and turning waves, with no dip limitations). In this study, vertical delineation of the plume was also somewhat limited due to the use of a band-limited wavelet $(<60 \mathrm{~Hz})$. Depth resolution in the seismic data was therefore approximately $25 \mathrm{~m}(\lambda / 4)$ in the reservoir. Based on the vertical distribution of the plume layers in Figure 7-7, this resolution should be adequate to differentiate between reservoir units. However, if the $3^{\text {rd }}$ perforation zone did in fact permit $\mathrm{CO}_{2}$ into the lower part of the upper Deadwood, then better resolution may be required to differentiate the $\mathrm{CO}_{2}$ plume in that horizon from the plumes in the over- and underlying units.

A limitation on the characterization of the plume in early stages of injection is brought upon by the size of the plume relative to the Fresnel zone. Sherriff and Geldart (1995) show that the migrated amplitudes for sub-Fresnel zone reflectors will be a 
fraction of those associated with a specular reflection that is proportional to the size of the reflector relative to the size of the Fresnel zone. For a zero-offset shot, the $50 \mathrm{~Hz}$ Fresnel radius is $\sim 325 \mathrm{~m}$ at the reservoir depth for the uppermost receiver, and $\sim 125 \mathrm{~m}$ for the lowermost. Therefore, the $36 \mathrm{kt}$ plume is smaller than the Fresnel zone for most receivers, while the $97 \mathrm{kt}$ plume is larger than the Fresnel zone for at least the lower receivers. This effect is exemplified when comparing the RMS of the time-lapse difference volumes for the synthetic $36 \mathrm{kt}$ and $97 \mathrm{kt}$ scenarios in Figure 7-12 and Figure 7-14. The fluid flow simulations yield similar saturation and P-wave contrast values for each scenario, however their seismic RMS magnitudes differ by a factor of $\sim 1.4$ near the injection well.

The existence of the specular blind-spot in the location of the plume appears to be mitigated by the wide aperture used for migration. The reservoir horizons in Figure 7-10 and the Deadwood anomalies in Figure 7-12 exhibit no amplitude decay directly below the sensors, validating the imaging methodology used in Harris et al. (2017).

There is a marked discrepancy in the magnitude of the seismic difference amplitudes (RMS or nRMS) between the synthetic and the field data volumes. As the workflow was effectively identical for the field and synthetic data, it is unlikely that this discrepancy resulted from the processing. It is also unlikely that it can be attributed to the flow simulation underestimating saturation, given that Figure 7-8 suggests that a \pm 0.10 change in saturation in the lower Deadwood would only yield a $\pm 1 \%$ change in P-wave impedance. It is more likely that the disagreement originates from the validity of assumptions made during Gassmann-based fluid substitution, particularly, those pertaining to the shale (illite) content in the lower Deadwood $(\sim 15 \%)$. 
The first assumption is that the rock is composed of constitutive minerals that have similar elastic properties. The Gassmann algorithm from Smith et al. (2003) approximates the pore compressibility as equal to the mineral grain compressibility, which is true for a homogenous medium, but not generally so for a heterogeneous medium (Brown and Korringa, 1975). Pore compressibility refers to the change in pore volume due to change in pore pressure for constant differential pressure, and can vary widely for sand-clay mixtures. Berge (1998) found that estimates of the saturated bulk modulus for sand-clay mixtures can change by a factor of 2 if the pore compressibility is controlled by the fluid compressibility instead of the mineral grain compressibility. It should be noted, however, that the Berea sandstones described in Berge (1998) were somewhat more porous (19-21\%) than the sandstones in the Aquistore reservoir (5-8\%) (Hart and Wang, 2010).

The second assumption is that the pore space is well connected. Dvorkin et al. (2007) propose using effective porosity for fluid substitution in shaley sandstones to better reflect the inability of $\mathrm{CO}_{2}$ to permeate the unconnected pores. Upon substituting the average effective porosity $(\sim 0.046)$ for total porosity in the lower Deadwood, the sensitivity plot in Figure 7-15 shows that, at $0.50 \mathrm{CO}_{2}$ saturation, the reflectivity increases from 0.35 to 0.55 . This increase is almost identical to the difference in the magnitude of the anomalies for the $36 \mathrm{kt}$ scenario, for field and synthetic data. 


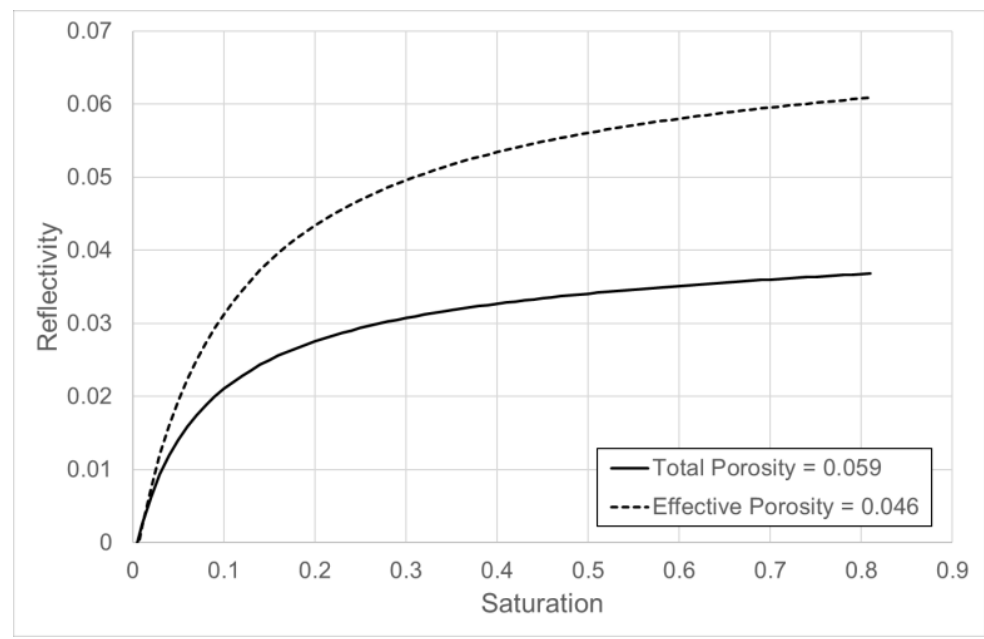

Figure 7-15: The effect on reflectivity of using the total versus effective porosity in the

Gassmann methodology. The reflectivity is computed at the boundary between the fluidsaturated lower Deadwood and the overlying shale unit.

It is also possible that the measurement errors on the log-based parameters significantly affect the fluid substitution results. In reality, a change in P-wave velocity is accompanied by a small increase in S-wave velocity, and the bulk modulus of the brinesaturated rock changes slowly with an increase in both velocities. However, since these velocities are measured independently, we will look at the case where measurement errors have caused one parameter to be over/underestimated without affecting the other. For the lower Deadwood $(>3300 \mathrm{~m})$, the formation P- and S-wave velocity averages are $4897 \pm 14 \mathrm{~m} / \mathrm{s}$ and $3043 \pm 13 \mathrm{~m} / \mathrm{s}$, respectively, taken over 336 samples. The formation density average is $2540 \pm 3 \mathrm{~kg} / \mathrm{m}^{3}$, taken over the same sample size. Figure 7-16 shows the sensitivity of Gassmann-derived P-wave velocity changes to the uncertainties in the above log-based parameters. The P-wave velocity changes are more sensitive to a per cent change in any of these parameters than they are to porosity. However the error ranges of $\mathrm{P}$-and $\mathrm{S}$-wave velocities, and density are fairly small and only amount to a 
maximum of $\pm 10 \%$ change in P-wave velocity contrast. Porosity values are also logbased, and are therefore subject to similar measurement errors. However, it is likely that this measurement error is smaller than the systematic error introduced by using total porosity for fluid substitution calculations.

The assumption that pore fluids mix homogeneously instead of heterogeneously (i.e. undergo "patchy" mixing) affects the bulk modulus of the saturated rock, and thus the impedance contrast. However, adjusting the model to account for a patchy brine- $\mathrm{CO}_{2}$ fluid mixture serves to reduce the impedance contrast for low saturations, and converges with the homogenous model for high saturations (Eid, 2015). Therefore the use of a patchy model would exacerbate the discrepancy between the synthetic and field anomalies. The addition of reservoir heterogeneity in future flow simulations, however, may increase the range of saturations in each unit, and might necessitate the use of a saturation distribution between the homogenous and patchy limits. 

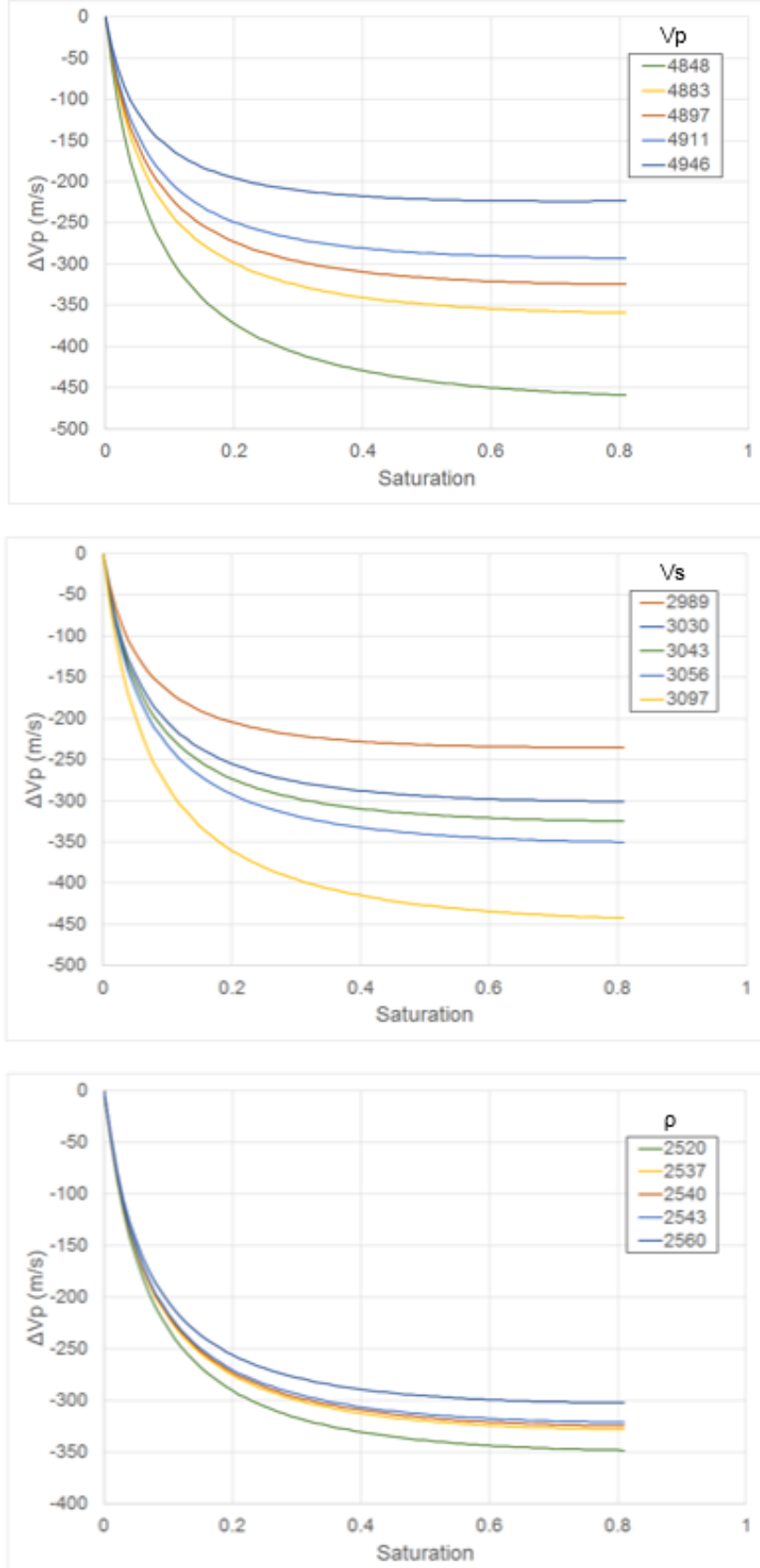

Figure 7-16: $\Delta \mathrm{Vp}$ sensitivity plots for log-based parameters in Gassmann fluid substitution calculations. In each plot, the middle line is the value estimated from the logs, the lines directly above and below it are the plus/minus one standard deviation of the mean measurements. 
Fluid flow simulations predict that most of the injected $\mathrm{CO}_{2}$ resides at high saturations within the upper and lower Deadwood formation $\left(2^{\text {nd }}\right.$ and $4^{\text {th }}$ perforation zones), assuming that the $3^{\text {rd }}$ perforation zone is closed off. Based on Gassmann's methodology, a more significant change in elastic properties is expected in the lower Deadwood: a 9\% decrease in $\mathrm{P}$-wave impedance at 0.60 saturation versus a $6 \%$ decrease in the upper Deadwood. The size of the plume is also predicted to be larger in the lower Deadwood than in the upper Deadwood. This results in a strong synthetic seismic anomaly located at $\sim 3300 \mathrm{~m}$ in both the RMS and nRMS difference in Figure 7-12. Based on the depth agreement with the field anomaly, these results suggest that the timelapse signal observed in the reservoir is primarily due to $\mathrm{CO}_{2}$ residing in the lower Deadwood at 3300-3330 m depth.

There is a degree of uncertainty in the depth of the anomaly introduced by the range of phase shifts that may be used to match the synthetic and field data. The phase of the field data in Figure 7-10 was aligned with that of the synthetic data by using the Icebox horizon as a reference. As a result, the wavelets near the top of the reservoir match well among the field and synthetic sections, but not quite as well near the bottom. Conversely, when the Precambrian basement horizon is used as the reference, the wavelets agree well near the bottom of the reservoir, but less so near the top. The effect is that the strongest point in the anomaly shifts in depth $<10 \mathrm{~m}$ depending on the horizon chosen for phase-matching. However, the anomaly remains centered in the lower Deadwood.

There is also a strong signal in the upper Deadwood $(\sim 3260 \mathrm{~m})$ in the nRMS section of the field data (Figure 7-12), however, that does not appear in the synthetic data, 
and cannot be explained singularly as a side-lobe of the band-limited wavelet from the lower Deadwood signal. If this were the case, then the $\sim 3260 \mathrm{~m}$ signal should be much weaker than the $\sim 3300 \mathrm{~m}$ signal, as is observed in the synthetic sections. However, when comparing the field and synthetic seismic sections in Figure 7-10, it can be seen that $\sim 3260 \mathrm{~m}$ is a particularly low-amplitude zone in the field section, and not so in the synthetic section. When normalizing the side-lobe energy by a low-amplitude in the field data, an exaggerated nRMS signal is produced here. Another possible explanation for the $\sim 3260 \mathrm{~m}$ signal is that the fluid flow simulations did not accurately characterize $\mathrm{CO}_{2}$ saturations in this unit. In the simulations, the $3^{\text {rd }}$ perforation zone was completely blocked-off on the basis of the spinner survey results indicating that no $\mathrm{CO}_{2}$ had been admitted into this zone. A possible reason for this blockage was that prior well testing may have induced clay swelling and reduced permeability near the injection well. The integrity of the spinner survey has since been questioned, introducing the possibility that some $\mathrm{CO}_{2}$ may be present in this zone. Based on previous elastic modeling of the lower part of the upper Deadwood in Roach et al. (2015), even low saturations in this zone can result in a sizeable seismic response. It is therefore possible that the signal at $\sim 3260$ in the field data in Figure 7-12 can be attributed to some quantity of $\mathrm{CO}_{2}$, but will necessitate further monitoring to confirm.

The anomaly in the basement visible in Figure 7-12 and Figure 7-13 highlights an issue with interpreting time-lapse differences exclusively in terms of nRMS. While computing the nRMS of an anomaly allows for a direct comparison with the limits imposed by unrepeatable effects, it does not distinguish between reflectivity changes and travel time changes resulting from the $\mathrm{CO}_{2}$ plume. In future analysis, this may be 
mitigated by applying time-variant shifts to re-align horizons in the migrated volumes before applying the nRMS window.

The fluid flow simulation predicted a laterally symmetrical plume expansion whereas the seismically observed plume trends N to NNW towards the observation well, with very little $\mathrm{CO}_{2}$ observed south of the injection well. The disagreement between the shape of the synthetic and the observed plumes was not unexpected as the flow simulations did not include any structure in the reservoir or basement. Future simulations will incorporate some mechanism(s) to account for the preferential migration direction, such as reservoir heterogeneity, anisotropic permeability, and basement topography.

\subsection{Conclusions and Outlook}

A workflow designed to model the evolution of the injected $\mathrm{CO}_{2}$ plume has been shown to produce seismic anomalies that are in partial agreement with those from the field surveys. However, it is clear that additional effort is required to better predict the shape and location of the plume for larger injection quantities.

Both the field and synthetic seismic volumes suggest that the strongest $\mathrm{CO}_{2}$-based anomalies are located in the lower Deadwood formation. Fluid replacement calculations show that this is the reservoir unit that exhibits the most pronounced decrease in seismic impedance with increasing $\mathrm{CO}_{2}$ saturation; and the fluid flow simulations predict that a significant quantity of $\mathrm{CO}_{2}$ has been permitted into this unit. However, there is a lowamplitude anomaly visible in the nRMS field data volume in the lower part of the upper Deadwood formation that is not visible in the corresponding synthetic volume. This anomaly is located near a well perforation zone that was closed-off in the flow 
simulations, and thus no $\mathrm{CO}_{2}$ was predicted to reside. A reassessment of the status of this perforation zone will have to wait until more field data is available.

This is an early iteration of the reservoir model, and as such, did not accurately reproduce the asymmetric expansion of the plume around the injection well. Subsequent models will require additional structure in the lower reservoir units and/or basement to understand the future trajectory of the plume.

\subsection{Acknowledgements}

The authors would like to thank the Geological Survey of Canada, and SaskPower. Brian Roberts and Lisa Roach are thanked for their helpful work in the field at Aquistore, as well as the Petroleum Technology Research Centre and Kyle Worth for project management. The seismic data were acquired by Silixa, Schlumberger Carbon Services, Tesla Exploration, Geospace Technologies and the Geological Survey of Canada. We would like to acknowledge Chris Hawkes for his helpful work with the simulation results. We would also like to thank Chevron for their contributions. 


\section{Conclusions}

\subsection{Summary}

This thesis presented three separate studies that, when taken together, demonstrate the ability to monitor injected $\mathrm{CO}_{2}$ in a deep geological reservoir using DAS VSP data. A site-specific feasibility study was undertaken prior to $\mathrm{CO}_{2}$ injection that sought to affirm that the expected plume response would be detectable through non-repeatable noise. A 4D DAS VSP dataset was then analyzed after $36 \mathrm{kt}$ of $\mathrm{CO}_{2}$ injection, and an anomaly in the Deadwood formation was identified and attributed to the presence of $\mathrm{CO}_{2}$ near the injection and observation wells. The interpretation of this anomaly was further refined with supplementary fluid flow replacement and seismic modeling results, and characterization of the size and location of the plume were performed.

It was demonstrated in the feasibility study that as little as $27 \mathrm{kt}$ of $\mathrm{CO}_{2}$ could be detected above the time-lapse noise of the surveys in the lower part of the reservoir, i.e., the Deadwood formation. Seismic responses were also simulated for 110, 220, and $330 \mathrm{kt}$ of injected $\mathrm{CO}_{2}$, each yielding an anomaly of increasing size and magnitude, but significantly more visible in the lower injection intervals. The visibility criterion suggests that an anomaly of nRMS $>0.3$ can be detected, which is not exceeded in the upper part of the reservoir, i.e., the Black Island member, until 110-220 kt of $\mathrm{CO}_{2}$ injection.

Analysis of time-lapse difference between the first monitor survey and the baseline survey, i.e. after $36 \mathrm{kt}$ of $\mathrm{CO}_{2}$ injection, highlighted an anomaly in the lower Deadwood extending north-northwest from the injection well. The time-lapse noise did not exceed a nRMS of 0.4 near the injection and observation wells, while the nRMS 
associated with the anomaly generally exceeded 0.7 . As predicted in the feasibility study, there was no detectable response in the Black Island member.

Seismic modeling was then performed with updated history-matched fluid flow simulations for $36 \mathrm{kt}$ and $97 \mathrm{kt}$ of injected $\mathrm{CO}_{2}$. The synthetic seismic data was processed with a functionally identical sequence to what was devised for the field data. The response of the modeled plume in the lower Deadwood was in close agreement with the response observed from the field data near the injection well, and thereby aided in further localization of the plume. However, disagreement in the lateral shape and trajectory between the field and synthetic anomalies necessitates further effort in refining the fluid flow simulations.

\subsection{Future work}

The repeatability achieved after processing and stacking the baseline and monitor survey data was adequate to make characterizations of the $36 \mathrm{kt} \mathrm{CO}_{2}$ plume. However, a further reduction of time-lapse noise in the difference images would add robustness to the interpretations presented in this thesis and future analyses. The use of the first monitor survey in lieu of the baseline would contribute to this objective in several ways. The SNR in the monitor data is higher, and thus the SNR in the difference images with subsequent monitor data would be higher. It is also likely that more shot gathers could be included in the stack, as many of the baseline shot gathers were excluded due to unacceptably high noise and significantly differing wavelets. As most of the excluded shot gathers were located at far offsets, more shots in the stack would also expand spatial coverage, which may be necessary as the plume expands laterally. Substituting the baseline data for the first monitor data would also serve to increase the bandwidth in the difference images, 
and consequently the vertical resolution in the reservoir, allowing more precise localization of the plume as a function of depth. The primary drawback of this substitution of survey vintages would be the loss of the $36 \mathrm{kt}$ plume response in subsequent difference images. Future time-lapse analyses will require that interpretations account for (either qualitatively or quantitatively) the corresponding loss of signal near the injection and observation wells.

More accurate characterization of the expanding plume will call for updated geological models and fluid flow simulations, the constructions of which will require an iterative process whereby reservoir models are history-matched to both injection data and seismic data. Model outputs must not only reflect flow parameters measured at the injection well, but also produce $\mathrm{CO}_{2}$ distributions that have calculated seismic and $\log$ responses consistent with those of the field data. Residual error between the synthetic and field seismic responses after each iteration will be used to refine the reservoir model for the next iteration.

\section{References}

Ahmadi, A.B., and Morozov, I., 2011, Time-Lapse VSP Data Analysis from Weyburn $\mathrm{CO}_{2}$ Project: Recovery - 2011 CSPG CSEG CWLS Convention.

Arts, R., Eiken, O., Chadwick, R.A., Zweigel, P., van der Meer, L., and Kirby, G.A., 2004, Monitoring of CO2 injected at Sleipner using timelapse seismic data: Energy, $29,1383-1392$. 
Arts, R., Chadwick, A., Eiken, O., Thibeau, S., and Nooner, S., 2008, Ten years' experience of monitoring $\mathrm{CO}_{2}$ injection in the Utsira Sand at Sleipner, offshore Norway: First Break, 26, 65-72.

Bacci, V.O., O’Brien, S., Frank, J., and Anderson, M., 2017, Using a Walk-away DAS Time-lapse VSP for $\mathrm{CO}_{2}$ Plume Monitoring at the Quest CCS Project: CSEG Recorder, 42 (3), 18-21.

Bachu, S., Gunter, W.D., and Perkins, E.H., 1994, Aquifer disposal of $\mathrm{CO}_{2}$ : hydrodynamic and mineral trapping: Energy Conversion and Management, 35(4), $269-279$.

Bachu, S., Faltinson, J., Hauck, T., Perkins, E., Peterson, J., Talman, S., and Jensen, G., 2011, The Basal Aquifer in the Prairie region of Canada - characterization for $\mathrm{CO}_{2}$ storage: Preliminary report for Stage I (Phases 1 and 2), Alberta Innovates Technology Futures.

Batzle, M., and Wang, Z., 1992, Seismic properties of pore fluids: Geophysics, 57, 1396 $-1408$.

Berge, P. A., 1998, Pore compressibility in rocks, in J. F. Thimus, Y. Abousleiman, A. H. Cheng, O. Coussy, and E. Detournay, eds., Poromechanics: A.A. Balkema, 351-356.

Birkholzer, J.T., Zhou, Q., Tsang, C., 2009, Large-scale impact of $\mathrm{CO}_{2}$ storage in deep saline aquifers: A sensitivity study on pressure response in stratified systems: International Journal of Greenhouse Gas Control, 3, 181-194. 
Bohlen, T., 2002, Parallel 3-D Viscoelastic Finite-Difference Seismic Modelling, Computers \& Geosciences, 28(8), 887-899.

Bostick III, F., 2000, Field experimental results of three-component fiber-optic sensors: 70th Ann. Internat. Mtg: Soc. of Expl. Geophys., Exp. Abstr., 21-24.

Bostick, F., 2011, Permanently installed in-well fiber optic accelerometer-based sensing apparatus and associated method: US Patent 8020436 B2. Washington, DC: U.S.

Botnen, L., D.W. Fisher, A.A. Dobroskok, T.R. Bratton, K.H. Greaves, T.R. McLendon, G. Steiner, J.A. Sorensen, E.N. Steadman, J.A. Harju, 2009, Field Test of $\mathrm{CO}_{2}$ Injection and Storage in Lignite Coal Seam in North Dakota: GHGT-9, Energy Procedia, 2013-2019.

Brown, R. J., and J. Korringa, 1975, On the dependence of the elastic properties of a porous rock on the compressibility of the pore fluid: Geophysics, 40, 608-616

Celia, M.A., S. Bachu, J.M. Nordbotten, S.E. Gasda and H.K. Dahle, 2005, Quantitative estimation of $\mathrm{CO}_{2}$ leakage from geological storage: Analytical models, numerical models and data needs: GHGT-7 Proceedings, 663-672.

Correa, J., Freifeld, B.M., Robertson, M., Daley, T.M., 2017, Distributed Acoustic Sensing Applied to 4D Seismic - Preliminary Results from the CO2CRC Otway Site Field Trials: 79th EAGE Conference and Exhibition.

Couëslan, M.L., Gulati, J., Campbell A., and Nutt, L., 2013, Monitoring $\mathrm{CO}_{2}$ Injection at the Illinois Basin -- Decatur Project with Time-lapse 3D VSPs: Second EAGE Workshop on Borehole Geophysics. 
Coueslan, M.L., Ali, S., Campbell, A., Nutt, W.L., Leaney, W.S., Finley, R.J., and Greenberg, S.E., 2013, Monitoring $\mathrm{CO}_{2}$ injection for carbon capture and storage using time-lapse 3D VSPs: The Leading Edge, 32, 1268-1276.

Cox, B., Wills, P., Kiyashchenko, D., Mestayer, J., Lopez, J., Bourne, S., Lupton, R., Solano, G., Henderson, N., Hill, D., and Roy, J., 2012, Distributed Acoustic Sensing for Geophysical Measurement, Monitoring and Verification: CSEG Recorder, 37 (2), 7-13.

Daley, T. M., Freifield, B.M., Ajo-Franklin, J., Dou, S., Pevzner, R., Shulakova, V. et al., 2013, Field testing of fibre-optic distributed acoustic sensing (DAS) for subsurface seismic monitoring: The Leading Edge 32(6), 699-706.

Daley, T.M., Robertson, M., Freifeld, B.M., White, D., Miller, D.E., Herkenhoff, F., and Cocker, J., 2014, Simultaneous acquisition of distributed acoustic sensing VSP with multi-mode and single-mode fiber optic cables and 3C-geophones at the Aquistore $\mathrm{CO}_{2}$ storage site: 85th Ann. Internat. Mtg: Soc. of Expl. Geophys., Exp. Abstr., 50145018.

Daley, T.M., Miller, D.E., Dodds, K., Cook, P., and Freifeld, B.M., 2015, Field testing of modular borehole monitoring with simultaneous distributed acoustic sensing and geophone vertical seismic profiles at Citronelle, Alabama: Geophysical Prospecting.

Daley, T.M., Miller, D.E., Dodds, K., Cook, P. and Freifeld, B.M., 2016, Field testing of modular borehole monitoring with simultaneous distributed acoustic sensing and geophone vertical seismic profiles at Citronelle, Alabama: Geophysical Prospecting, 64, 1318-1334. 
Dillon, P.B. and Thomson, R.C., 1984, Offset source VSP surveys and their image reconstruction: Geophysical Prospecting, 32, 790-811.

Dillon, P.B., 1988, Vertical seismic profile migration using the Kirchhoff Integral: Geophysics, 53(6), 786-799.

Duxbury, A., D. White, C. Samson, S. Hall, J. Wookey, and J.M. Kendall, 2012. Fracture mapping using AVOA analysis at the Weyburn $\mathrm{CO}_{2}$ storage site. Geophysics, 77(6): N17-N28.

Dvorkin, J., Mavko, G., and Gurevich, B., 2007, Fluid substitution in shaley sediment using effective porosity: Geophysics, 72 (3), O1-O8.

Eid, R., Ziolkowski, A., Naylor, M, Pickup, G., 2015, Seismic monitoring of $\mathrm{CO}_{2}$ plume growth, evolution and migration in a heterogeneous reservoir: Role, impact and importance of patchy saturation, In International Journal of Greenhouse Gas Control, $43,70-81$.

Energy and Environmental Research Center (EERC), 2014, Geologic modeling and simulation report for the Aquistore project, Plains $\mathrm{CO} 2$ reduction (PCOR) partnership phase III task 1 - Deliverable D93.

Energy and Environmental Research Center (EERC), 2016, Geologic modeling and simulation report for the Aquistore project, Plains $\mathrm{CO} 2$ reduction (PCOR) partnership phase III task 1 - Deliverable D93, Update 2.

Freifeld, B.M., R. Pevzner, S. Dou, T. Daley, M. Robertson, K. Tertyshnikov, T. Wood, J. Ajo-Franklin, M. Urosevic, B. Gurevich, The CO2CRC Otway Project deployment 
of a Distributed Acoustic Sensing Network Coupled with Permanent Rotary Sources: 78th EAGE Conference \& Exhibition 2016, EAGE, Vienna, Austria, 2016, Tu LHR2 06.

Gassmann, F., 1951, Elastic waves through a packing of spheres: Geophysics, 16(4), 673.

Ghorbani, A., Zamora, M., Cosenza, P., 2009, Effects of desiccation on the elastic wave velocities of clay-rocks: International Journal of Rock Mechanics and Mining Sciences, 46, 1267-1272.

Götz, J., Lüth, S., Krawczyk, C.M., and Cosma, C., 2014, Zero-Offset VSP Monitoring of CO2 Storage: Impedance Inversion and Wedge Modelling at the Ketzin Pilot Site: International Journal of Geophysics, 2014.

Hardage, B.A., 2000, Vertical Seismic Profiling: Principles. Oxford: Elsevier Science Ltd.

Harris, K., White, D., Samson, C., Daley, T., and Miller, D.E., 2015, Evaluation of distributed acoustic sensing for 3D time-lapse VSP monitoring of the Aquistore $\mathrm{CO}_{2}$ storage site: 2015 GeoConvention: New Horizons. Exp. Abstr.

Harris, K., D. White, D. Melanson, C. Samson, and T. Daley, 2016, Feasibility of timelapse VSP monitoring at the Aquistore $\mathrm{CO}_{2}$ storage site using a distributed acoustic sensing system: International Journal of Greenhouse Gas Control, 50, 248-260.

Harris, K., D. White, C. Samson, 201x, Imaging the Aquistore reservoir after 36 kilotonnes of $\mathrm{CO}_{2}$ injection using distributed acoustic sensing: Geophysics (accepted). 
Harvey, A.H., 1996, Semiempirical correlation for Henry's Constants over large temperature ranges: American Institute of Chemical Engineers Journal, 42, 1491.

Hole, J.A. and Zelt, B.C., 1995, 3-D finite-difference reflection travel times: Geophysical Journal International, 121(2), 427-434.

Hornby, B.E., Burch, T., 2008, Passive drive by imaging in a deep water production well using permanent borehole seismic sensors: 78th Ann. Internat. Mtg., Soc. Expl. Geophys., Exp. Abstr., 349-352.

Hornman, K., 2016, Field trial of seismic recording using distributed acoustic sensing with broadside sensitive fibre-optic cables: Geophys. Prospect, doi: 10.1111/13652478.12358.

Huang, X., Meister, L., and Workman, R., 1998, Improving production history matching using time-lapse seismic data: The Leading Edge, 17(10), 1430-1433.

IPCC (Intergovernmental Panel on Climate Change), 2005, IPCC special report on carbon dioxide capture and storage: Prepared by Working Group III of the Intergovernmental Panel on Climate Change. Cambridge University Press, Cambridge.

Ivandic, M., Juhlin, C., Lueth, S., Bergmann, P., Kashubin, A., Sopher, D., Ivanova, A., Baumann, G. and Henninges, J., 2015, Geophysical monitoring at the Ketzin pilot site for $\mathrm{CO}_{2}$ storage: New insights into the plume evolution: International Journal of Greenhouse Gas Control, 32, 90-105. 
Kestin, J., Khalifa, H.E., and Correia, R.J., 1981, Tables of dynamic and kinematic viscosity of aqueous $\mathrm{NaCI}$ solutions in the temperature range $20^{\circ}-150^{\circ} \mathrm{C}$ and the pressure range 0.1-35 MPa: Journal of Physical and Chemical Reference Data, v. 10, p. 71-87.

Komatitsch, D., and Martin, R., 2007, An unsplit convolutional perfectly matched layer improved at grazing incidence for the seismic wave equation: Geophysics, 72(5), 155 $-167$.

Kragh, E., and Christie, P., 2002, Seismic repeatability, normalized rms, and predictability: The Leading Edge, 21, 640-647.

Kuvshinov, B.N., 2016, Interaction of helically wound fibre-optic cables with plane seismic waves: Geophysical Prospecting, 64, 671-688.

Kuzmiski, L., Charters, B., and Galbraith, M., 2009, Processing considerations for 3D VSP: CSEG Recorder, Focus Article, 34(4).

Levander, A.R., 1988, Fourth-order finite-difference P-SV seismograms: Geophysics, 53 (11), 1425-1436.

Li, X., K. Lazorko, P. Cary, and G. Margrave, 2011, Simultaneous time-lapse processing for optimal repeatability: CREWES Research Report, 23.

Lumens, P., A. Franzen, K. Hornman, S.G. Karam, G. Hemink, B. Kuvshinov, J. La Follett, B. Wyker, and P. Zwartjes, 2013, Cable development for distributed geophysical sensing with a field trial in surface seismic: Presented at the Fifth 
European Workshop on Optical Fibre Sensors, International Society for Optics and Photonics, 879435.

Lumley, D. E., 2001, Time-lapse seismic reservoir monitoring: Geophysics, 66(1), 5053.

Lumley, D. E., D. C. Adams, M. A. Meadows, and S. P. Cole, 2003, 4D seismic data processing issues and examples: 73rd Annual International Meeting, SEG, Expanded Abstracts, 1394-1397.

Lumley, D. E., D.C. Adams, R.J. Wright, D. Markus, and S.P. Cole, 2008, Seismic monitoring of $\mathrm{CO}_{2}$ geo-sequestration - realistic capabilities and limitations: 78th Annual International Meeting, SEG, Expanded Abstracts, 27-1, 2841-2845.

Lumley, D. E, 2009, 4D Seismic Monitoring of Subsurface Fluid Flow: CSEG Recorder, $34,8$.

Mateeva, A., J. Lopez, H. Potters, J. Mestayer, B. Cox, D. Kiyashchenko, P. Wills, S. Grandi, K. Hornman, B. Kuvshinov, W. Berlang, Z. Yang, and R. Detomo, 2014, Distributed acoustic sensing for reservoir monitoring with vertical seismic profiling: Geophysical Prospecting, 62, 679-692.

Melanson, D., and White, D., 2015, Model assembly, Gassmann fluid substitution and 3D finite difference seismic simulations at the Aquistore $\mathrm{CO}_{2}$ injection site, Estevan, Saskatchewan. Geological Survey of Canada, Natural Resources Canada.

Mestayer, J. Cox, B., Wills, P., Kiyashchenko, D., Lopez, J., Costello, M., Bourne, S., Ugueto, G., Lupton, R., and Solano, G., 2011, Field trials of distributed acoustic 
sensing for geophysical monitoring: 81st Ann. Internat. Mtg: Soc. of Expl. Geophys., Exp. Abstr., 4253-4257.

Miller, D., Oristaglio, M., and Beylkin, G., 1987, A new slant on seismic imaging: Migration and integral geometry: Geophysics, 52 (7), 943-964.

Miller, D.E., Parker, T., Kashikar, S., Todorov, M. and Bostick, T., 2012, Vertical seismic profiling using a fiber-optic cable as a distributed acoustic sensor: 74th EAGE Conference and Exhibition.

Miller, D.E., T.M. Daley, D. White, B.M. Freifeld, M. Robertson, J. Cocker, M. Craven, 2016, Simultaneous acquisition of distributed acoustic sensing VSP with multi-mode and single-mode fibre-optic cables and $3 \mathrm{C}$-geophones at the Aquistore $\mathrm{CO}_{2}$ storage site: CSEG Recorder, 41, no. 6.

Ning, I.L.C. and P. Sava, 2016, Multicomponent Distributed Acoustic Sensing: in 86th Annual International Meeting, SEG, Expanded Abstracts, 5597-5602.

Nguyen, P.K.T., M.J. Nam, and C. Park, 2015, A review on time-lapse seismic data processing and interpretation: Geosciences Journal, 19, no.2, 375-392.

O'Brien, J., Kilbride, F., and Lim, F., 2004, Time-lapse VSP reservoir monitoring: Geophysics, 23(11), 1178-1184.

Parker, T., Shatalin, S., and Farhadiroushan, M., 2014, Distributed Acoustic Sensing - a new tool for seismic applications: First Break, 32, 61-69. 
Peck, W., Klenner, R., Liu, G., Gorecki, C., and Steadman, E., 2014, Geologic modeling and simulation report for the Aquistore project: Plains $\mathrm{CO}_{2}$ Reduction (PCOR) Partnership Phase III, Task 1 - Deliverable D93, Technical Report, March 2014.

Ringrose, P.S, Mathieson, A.S., Wright, I.W., Selama, F., Hansen, O., Bissell, R., Saoula, N., Midgley, J., 2013, The In Salah $\mathrm{CO}_{2}$ Storage Project: Lessons Learned and Knowledge Transfer: Energy Procedia, 37, 6226-6236.

Roach, L.A.N., White, D., and Roberts, B., 2014, An assessment of the time-lapse seismic repeatability using a permanent for reservoir monitoring at the Aquistore $\mathrm{CO}_{2}$ Storage Site, Saskatchewan, Canada: 84th Ann. Internat. Mtg: Soc. of Expl. Geophys., Exp. Abstr., 4924-4929.

Roach, L.A.N., White, D.J., and Roberts, B., 2015, Assessment of 4D seismic repeatability and $\mathrm{CO}_{2}$ detection limits using a sparse permanent land array at the Aquistore $\mathrm{CO}_{2}$ storage site: Geophysics, 80 (2), WA1-WA13.

Roach, L.A.N., White, D.J., and Roberts, B., Angus, D., 2017, Initial 4D seismic results after $\mathrm{CO}_{2}$ injection start-up at the Aquistore storage site: Geophysics, 82 (3), 1-13.

Rostron, B., White, D., Hawkes, C., Chalaturnyk, R., 2014, Characterization of the Aquistore $\mathrm{CO}_{2}$ project storage site, Saskatchewan, Canada: Energy Procedia 63, 2977 $-2984$.

Rowe, A.M., and Chou, J.C.S., 1970, Pressure-volume-temperature-concentration relation of aqueous $\mathrm{NaCl}$ solutions: Journal of Chemical Engineering Data, 15 (1), $61-66$. 
Saito, H., Nobuoka, D., Azuma, H., Tanase, D., Xue, Z., 2008, Time-Lapse Cross-Well Seismic Tomography for Monitoring $\mathrm{CO}_{2}$ Geological Sequestration at the Nagaoka Pilot Project Site: Journal of MMIJ, 124 (1), 78-86.

Schneider W.A., 1978, Integral formulation for migration in two and three dimension: Geophysics, 43(1), 49-76.

Shatalin, S., Treschikov, V., and Rogers, A., 1998, Interferometric optical time-domain reflectometry for distributed optical-fiber sensing: Appl. Opt., 37, 5600-5604.

Sheriff, R.E., and L.P. Geldart, 1995, Exploration Seismology (2nd Ed.). Cambridge: Cambridge UP.

Smith, T.M., Sondergeld, C.H., and Rai, C.S., 2003, Gassmann fluid substitutions: A tutorial.

Sorensen, J.A., D.D. Schmidt, D.J. Knudsen, S.A. Smith, C.D. Gorecki, E.N. Steadman, and J.A. Harju, 2011, Northwest McGregor field CO2 Huff 'n' Puff: A case study of the application of field monitoring and modeling techniques for $\mathrm{CO}_{2}$ prediction and accounting : in 10th International Conference on Greenhouse Gas Control Technologies, GHGT-10, Energy Procedia, 4, 3386-3393.

Thomsen, L., 1986, Weak elastic anisotropy: Geophysics 51 (10), 1954-1966.

Urosevic, M., Pevzner, R., Kepic, A., Wisman, P., Shulakova, V., and Sharma, S., 2010, Time-lapse seismic monitoring of $\mathrm{CO}_{2}$ injection into a depleted gas reservoir Naylor Field, Australia: The Leading Edge, 29, 164-169. 
Verdon, J.P., 2012, Microseismic Monitoring and Geomechanical Modelling of $\mathrm{CO}_{2}$ Storage in Subsurface Reservoirs: Springer Theses, Springer-Verlag Berlin Heidelberg.

Virieux, J., 1986, P-SV wave propagation in heterogeneous media: velocity-stress finite difference method: Geophysics, 51(4), 889-901.

Wang, Z., 2001, Fundamentals of seismic rock physics: Geophysics, 66, 398-412.

White, D.J., G. Burrowes, T. Davis, Z. Hajnal, K. Hirsche, I. Hutcheon, E. Majer, B. Rostron, and S. Whittaker, 2004, Greenhouse gas sequestration in abandoned oil reservoirs: International Energy Agency Weyburn pilot project GSA Today, 14, 4-10.

White, D., 2009, Monitoring $\mathrm{CO}_{2}$ storage during EOR at the Weyburn-Midale Field: The Leading Edge, 28(7), 838-842.

White, D., 2013, Seismic characterization and time-lapse imaging during seven years of $\mathrm{CO}_{2}$ flood in the Weyburn field, Saskatchewan, Canada: International Journal of Greenhouse Gas Control, 16, S78-S94.

White, D.J., Roach, L.A.N., Roberts, B., 2015, Time-lapse seismic performance of a sparse permanent array: Experience from the Aquistore $\mathrm{CO}_{2}$ storage site: Geophysics, 80 (2), WA35-WA48.

White, D.J., Hawkes, C.D., and Rostron, B.J., 2016, Geological characterization of the Aquistore $\mathrm{CO}_{2}$ storage site from 3D seismic data: International Journal of Greenhouse Gas Control, 54(1), 330-344. 
Whittaker, S. and Worth, K., 2011, Aquistore: a fully integrated demonstration of the capture, transportation and geologic storage of $\mathrm{CO}_{2}: 10$ th Internat. Conf. on Greenhouse Gas Control Technologies, Energy Procedia: 4, 5607-5614.

Wood, A.B., 1964, A Textbook of Sound: Bell and Sons, London.

Worth, K., White, D., Chalaturnyk, R., Sorensen, J., Hawkes, C., Rostron, B., Johnson, J., Young, A., 2014, Aquistore Project Measurement, Monitoring, and Verification: From Concept to $\mathrm{CO}_{2}$ Injection: GHGT-12, Energy Procedia 63, $3202-3208$.

Worth, K., D. White, R. Chalaturnyk, J. Sorensen, C. Hawkes, B. Rostron, D. Risk, A. Young, N. Sacuta, 2017, Aquistore: Year One - Injection, Data, Results: Energy Procedia, 114, 5624-5635.

Wright, R., F. Mourits, L.B. Rodríguez, M.D. Serrano, 2013, The first North American carbon storage atlas: GHGT-11, Energy Procedia, 37, 5280 - 5289.

Wyatt, K. and Wyatt, S.B., 1981, Determination of subsurface structural information using the vertical seismic profile: Presented at the 51st Ann. Internat. Mtg., Soc. Expl. Geophys.

Yilmaz, O., 1987, Seismic data analysis: Processing, inversion, and interpretation of data, Volume 1. Society of Exploration Geophysicists.

Zhu, X. and McMechan, G. A., 1990, Direct estimation of the bulk modulus of the frame in a fluid-saturated elastic medium by Biot theory: 60th Ann. Internat. Mtg., Soc. Exp. Geophys., Expanded Abstracts. 787-790. Geophysics 68(2), 430 - 440. 